INTER NATIONAL MONETARY FUND
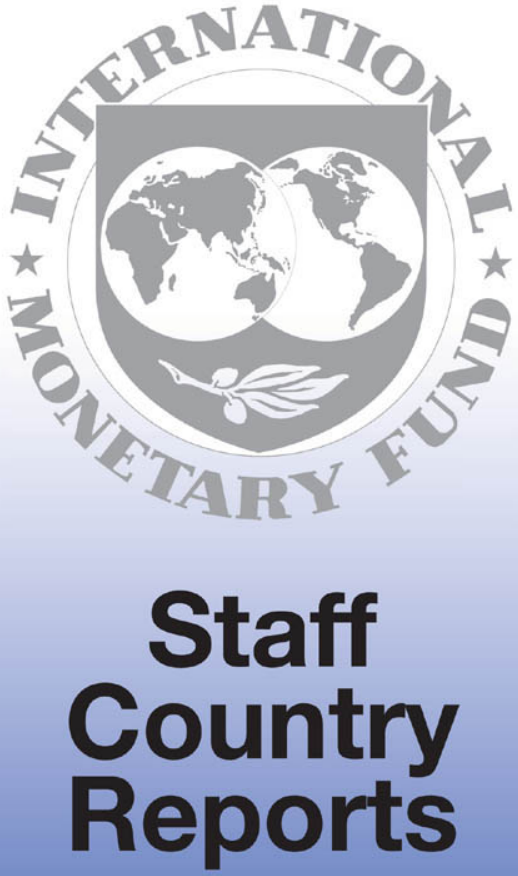


\section{Panama: Selected Issues and Statistical Appendix}

This Selected Issues and Statistical Appendix report on Panama was prepared by a staff team of the International Monetary Fund as background documentation for the periodic consultation with this member country. As such, the views expressed in this document are those of the staff team and do not necessarily reflect the views of the Government of Panama or the Executive Board of the IMF.

Copies of this report are available to the public from

International Monetary Fund - Publication Services 700 19th Street, N.W. - Washington, D.C. 20431

Telephone: (202) 623-7430 - Telefax: (202) 623-7201

Telex (RCA): 248331 IMF UR

E-mail: publications@imf.org

Intemet: http://www.imf.org

Price: $\$ 15.00$ a copy

\section{International Monetary Fund}

Washington, D.C. 


\section{INTERNATIONAL MONETARY FUND}

\section{PANAMA}

\section{Selected Issues and Statistical Appendix}

Prepared by G.A. Mackenzie (Head), J. Bailen, G. Bindley-Taylor, A. Gomez-Oliver, C. Keller, R. Randall, and M. Torres (all WHD)

Approved by the Western Hemisphere Department

February 1,2000

Contents

Page

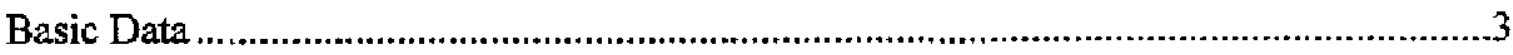

1. Social Security System-Current Trends and Directions for Reform........................

A. Basic Characteristics of the System ...........................................................

B. The CSS's Financial Situation ...................................................................6

C. The Complementary Public Pension Scheme FCPS and its Conversion

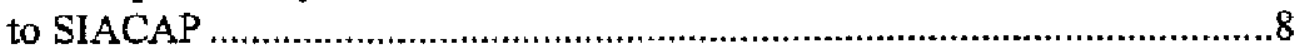

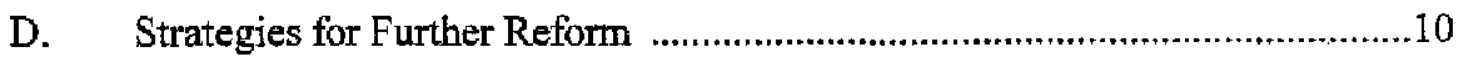

Text Tables

1. CSS Pension Benefits

2. Demographic Development ...........................................................................

Statistical Appendix Tables

3. National Accounts by Productive Activity .....................................................12

4. National Accounts by Use ........................................................................14

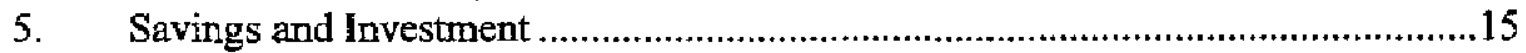

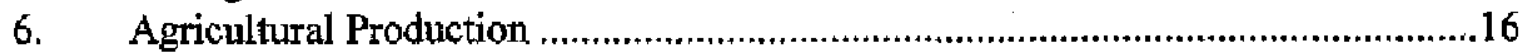

7. Value-Added in Manufacturing …..........................................................17

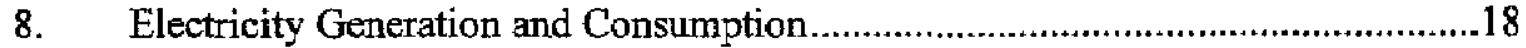

9. Private Sector Construction Permits, District of Panama .......................................19

10. Domestic Sales of Petroleum ....................................................................20

11. Labor Force Statistics ..................................................................................21

12. Average Prices Received by Farmers ..........................................................22

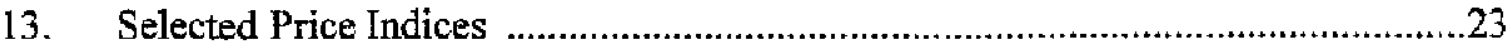

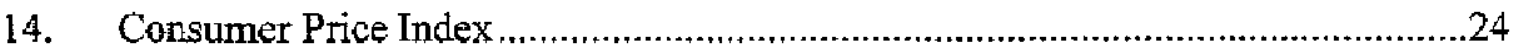

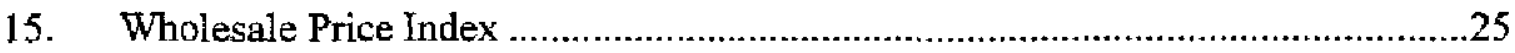

16. Selected Energy Prices.................................................................................26

17. Operations of the Nonfinancial Public Sector.....................................................27 


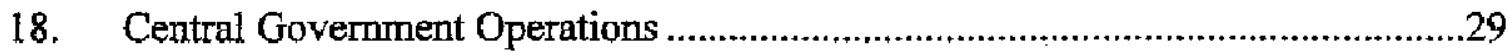

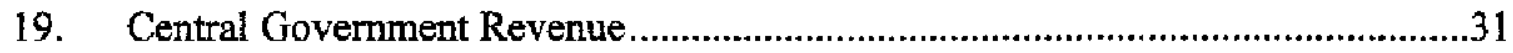

20. Central Government Expenditure ....................................................................32

21. Operations of the Social Security Agency …................................................33

22. Operations of the Decentralized Agencies ...........................................................34

23. Operations of the Public Enterprises...................................................................35

24. Nonfinancial Public Sector Gross Domestic Debt...............................................36

25. Accounts of the Banking System ........................................................................

26 . Summary Operations of the Banking System .............................................40

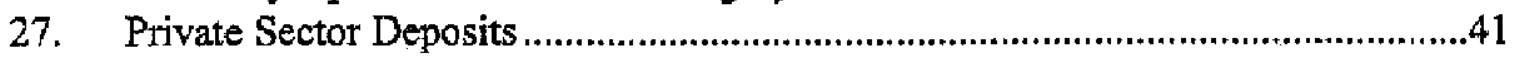

28. Offshore Operations of Private Banks ..............................................................42

29. Interest Rate Structure ................................................................................43

30. Banking System Credit to the Domestic Private Sector by Economic Activity .....44

31. Public Sector Banks-Operating Revenue and Expenditure..................................45

32. Public Sector Banks-Portfolio in Arrears..................................................47

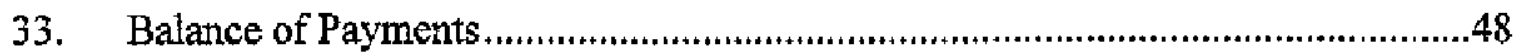

34. Summary Balance of Payments ……...........................................................5

35. Composition of Merchandise Exports …….....................................................51

36. Petroleum Trade ............................................................................................52

37. Nontraditional Exports and Issue of Tax Credit Certificates..................................53

38. Composition of Merchandise Imports, (c.i.f.)....................................................54

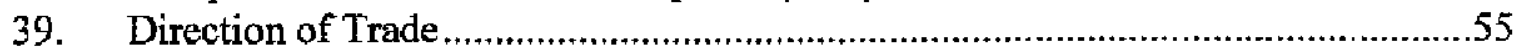

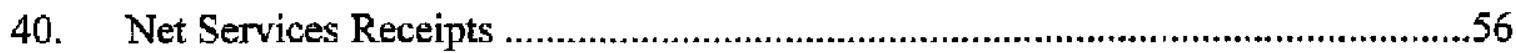

41. Operations of the Colon Free Zone ...............................................................57

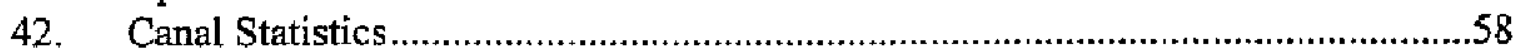

43. Principal Commodities Shipped Through the Canal ...........................................59

44. Travel Receipts and Expenditure, and Number of Visitors ................................60

45. Public Sector, External Debt and Debt Service ....................................................61

46. External Public Debt Indicators ....................................................................62

47. Operations on Medium- and Long-term External Debt of the Nonfinancial

Public Sector Classified by Creditor...............................................................63

48. Arrears on Public Sector External Debt Service ....................................................66 


\section{Panama: Basic Data}

\section{Social and Demographic Indicators}

Area (thousand sq. km)
Population (1999)
Total (million)
Rate of increase (percent a year)
Density (per sq. km.)
GDP per capita (US\$) (1999)
Unemployment (Aug. 1999)
Population eharacteristicg (1996)
Life expectancy at birth (years)
Crude birth rate (per thousand)
Crude death rate (per thousand)
Infant mortality (per thousand live birtins)
Income distribution (1995)
By highest 20 percent of households
By lowest 20 percent of households

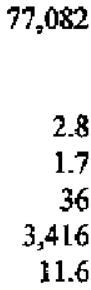

73.8

22.1

4.9

21.8

60

2
Health (1994)

Per capitai calorie intake Population

Per physician

Per hospital bed

(1992)

2,534

808

Access to sare water (percent of population, 1996)

Total
Urban
Rural
Education (in percent, 1996)
Secondary education
enrollment rates

82

84

68

68

GDP (1999)

US\$9,604 million

\section{Economic Indicators}

(In percent changes)

National accerunts and prices

GDP at constant 1982 market prices

Agriculture and mining

Manufacturing and construction

Services

Consumer price index (end-of-period)

Monetary $1 /$

Net domestic assets

Credit to the public sector

Credit to the private sector

Liabilities to the domestic private sector

Real effective exchange rate 2 /

$\begin{array}{rrrrr}1.8 & 2.8 & 4.5 & 4.1 & 3.5 \\ 3.1 & 0.8 & 1.2 & 3.3 & 7.2 \\ 1.3 & -2.1 & 6.1 & 4.1 & 0.1 \\ 1.6 & 4.2 & 4.8 & 4.0 & 3.5 \\ 0.8 & 2.3 & -0.5 & 1.4 & 1.5 \\ & & & & \\ 6.9 & 7.3 & 9.4 & 17.0 & 15.1 \\ -3.9 & 2.5 & -2.0 & -1.3 & -0.2 \\ 11.9 & 7.0 & 12.5 & 21.9 & 16.2 \\ 8.2 & 8.1 & 15.3 & 12.2 & 11.2 \\ -4.1 & 0.5 & 1.0 & 0.5 & 1.5\end{array}$

(In percent of GDP) $3 /$

\section{Investment and saving}

Gross investment

Private itivestratent

Guóss national saving

Private saving

30.2

26.8

25.6

22.3

29.2

29.0

25.6

3.4

5.6

0.2

Primary balance

Overall balance

Nonfinaneial public sector

Revenue and grants

Current

\section{External current account}

Gross public debt

External

Domestic

$\begin{array}{rrrrr}-4.7 & -3.7 & -6.9 & -13.3 & -13.8 \\ 86.5 & 77.2 & 71.9 & 69.3 & 73.2 \\ 74.5 & 62.0 & 57.8 & 58.1 & 58.4 \\ 12 & 15.2 & 14.1 & 11.4 & 14.8\end{array}$

35.6

29.4

21.8

17.6

27.5

23.7

3.8

3.8

31.1

34.3

27.9

$21.1 \quad 18.5$

$\begin{array}{lll}28.7 & 28.3 & 30.2 \\ 28.9 & 31.3 & 31.9\end{array}$

$\begin{array}{rrr}24.5 & 24.9 & 25.7 \\ 4.3 & 6.4 & 6.2\end{array}$

4.3

$\begin{array}{rrr}3.7 & 0.8 & 3.3 \\ -0.2 & -3.0 & -1.7\end{array}$

CInternational Monetary Fund. Not for Redistribution 
Panama: Basic Data

\begin{tabular}{|c|c|c|c|c|c|}
\hline & 1995 & 1996 & 1997 & 1998 & $\begin{array}{r}\text { Est. } \\
1999\end{array}$ \\
\hline \multicolumn{6}{|c|}{ (In millions of U.S. dollars) } \\
\hline \multicolumn{6}{|l|}{ Balance of payments } \\
\hline Trade balance & -588.9 & -644.1 & .700 .3 & $-1,371.1$ & $-1,464.1$ \\
\hline Exports, fo.b. & 744.4 & 740.6 & 844.3 & 864.2 & 825.8 \\
\hline linports, f.o.b. & $-1,997.4$ & $-2,248.9$ & $-2,462.2$ & $-2,736.9$ & $-2,840.0$ \\
\hline Colon free zone (net) & 664 & 864.2 & 917.6 & 501.6 & 550.1 \\
\hline $\begin{array}{l}\text { Services, income, and transfers (net) } \\
\text { Of which: }\end{array}$ & 219.8 & 342.2 & 96.8 & 159.4 & 134.7 \\
\hline Interest on pubiic debt & .393 .2 & -243.3 & -282.3 & -303.2 & -396.9 \\
\hline Cursent aceount & -369.1 & .301 .9 & -603.5 & $-1,211.7$ & $-1,329.4$ \\
\hline \multicolumn{6}{|l|}{ Capital and finencial aceount balance } \\
\hline (includigg errors and omissions) & 115.9 & 601.0 & 746.0 & 988.1 & $1,214,9$ \\
\hline Public (including grants) & -68.7 & -108.4 & -433.8 & -89.5 & 37.2 \\
\hline Private $=0-10$ & 209.6 & 879.8 & $1,435.9$ & $1,045.9$ & 848.8 \\
\hline Errors and omissions & -25.0 & -154.3 & -378.7 & $-195,0$ & 0.0 \\
\hline Overall balance & -253.2 & 299.1 & 142.6 & -223.5 & -114.4 \\
\hline Stock of net foreign assets of BNP & 708.7 & 952.1 & $1,082.3$ & 906.3 & 799.5 \\
\hline Stock of external payments arrears & $3,938.1$ & 92.8 & 41.2 & 0.0 & 0.0 \\
\hline \multicolumn{6}{|l|}{ Memorandum item: } \\
\hline Debt-service ratio $4 /$ & 22.4 & 15.2 & 44.3 & 19.8 & 25.4 \\
\hline \multicolumn{6}{|l|}{ IMF data (as of December 31, 1999) } \\
\hline \multicolumn{6}{|l|}{ Article VII status } \\
\hline \multicolumn{4}{|l|}{ Intervention currency and rate } & \multicolumn{2}{|c|}{ B 1.00 per U.S. dollar } \\
\hline \multicolumn{4}{|l|}{ Quota } & \multicolumn{2}{|c|}{ SDR 206.6 million } \\
\hline \multicolumn{4}{|l|}{ Fund holdings of local currency } & \multirow{2}{*}{\multicolumn{2}{|c|}{$\begin{array}{l}\text { SDR } 303.1 \text { million } \\
\text { SDR } 178.9 \text { million }\end{array}$}} \\
\hline \multicolumn{4}{|l|}{ From Fund resources (including SBA and EFF) } & & \\
\hline \multicolumn{4}{|l|}{ Fund holdings as percent of gliota } & \multirow{2}{*}{\multicolumn{2}{|c|}{$\begin{array}{l}146.7 \\
\text { None }\end{array}$}} \\
\hline \multicolumn{4}{|l|}{ Arrears with the Fund } & & \\
\hline \multicolumn{6}{|l|}{ Special Drawing Rights Department } \\
\hline Net Cumulative SDR allocation & & & & \multirow{2}{*}{\multicolumn{2}{|c|}{$\begin{array}{l}\text { SDR } 26.3 \text { million } \\
\text { SDR } \text { 1.2 million }\end{array}$}} \\
\hline Holdings of SDRs & & & & & \\
\hline
\end{tabular}

Sources: Panamanian authorities; and Fund staff estimates.

1/ In percent of initial stock of fiabilities to the private sector.

2/Based on period average.

3/ Reflects the authorities' revisions to nominal GDP for 1996 and 1997.

4/ External debt service as percent of exports of goods and serviees. In 1997 the increase reflects debt restructuring involving the retirement of Brady bonds. 


\section{Social SECurity System-CuRRent TRENDS AND DIRECTIONS For Reform}

\section{A. Basic Characteristics of the System}

1. Panama's social security system consists of the Social Security Agency (Caja de Seguro Social or CSS) and several complementary schemes. The CSS covers all private and public workers, while the complementary schemes provide additional benefits to selected groups. The civil servants' pension fund Fondo Complementario de Prestaciones Sociales(FCPS) has in the past been the largest of the complementary schemes; it has recently undergone a fundamental reform (Section C).

2. The CSS consists of several programs that cover the risks of old age, sickness, disability and occupational hazard. These are the old-age and disability pension program, Programa de Invalidez, Vejez y Muerte (IVM), the health program, Programa de Enfermedad y Maternidad (EM), and a program covering the risk of work accidents, Programa de Riesgos Profesionales (RP).

3. The CSS's coverage extends to about 60 percent of Panama's population, and includes 550,000 active contributors, 107,000 pensioners, and 980,000 dependents. About half of the economically active population contributes to the CSS. The number of private sector contributors has been growing less rapidly recently than private sector employment, so that the private sector coverage rate has fallen. The same is true of the public sector. ${ }^{1}$

4. The CSS is financed through payroll taxes (contributions) whose combined rate exceeds 20 percent. Contributions are paid by employees and employers at the rate of 9.5 percent (2.8 percent employer and 6.8 percent employee) for the IVM program; and 8.5 percent ( 8.0 percent employee and 0.5 percent employer) for the EM program. The average contribution rate for the RP program varies between 0.9 and 5 percent according to the risk category, and there are a few other minor levies.

5. Eligibility for a retirement pension requires that the male contributors have attained the age of 62 years. The minimum pensionable age for women is 57 years. In addition, a plan participant must have made at least 180 monthly contributions (over a minimum period of 15 years).

6. Pension levels are generous. The pensionable base, the reference wage, is defined as the average of the best seven years of a worker's wage history. For the minimum contribution period of 15 years a worker receives a pension of 60 percent of the reference wage; for every additional year of contribution payments $1 \frac{1 / 4}{4}$ percentage points are added. Thus, as shown in the table below, a person who has paid contributions over a working life of 40 years reaches a

${ }^{1}$ Between 1991-97, the rates of growth were as follows: private sector employment 7.0 percent; private sector contributors 5.6 percent; public sector employment 2.5 percent; public sector contributors 1.4 percent. 
replacement level of above 90 percent of the reference wage. Although a ceiling of US\$1,500 per month applies to pensions, the ceiling is not binding in practice on most pensioners, given that the average monthly wage is less than US\$540. When measured in terms of the average Panamanian wage the CSS's average replacement rates are 89 percent for old-age pensions, 68 percent for disability pensions, and 36 percent for survivor benefits.

Table 1. Panama: CSS Pension Benefits

\begin{tabular}{lrrrrrr} 
Years of contribution & 15 & 20 & 25 & 30 & 35 & 40 \\
\hline $\begin{array}{l}\text { Replacement rate } \\
\text { (In percent of reference wage) }\end{array}$ & 60.00 & 66.25 & 72.50 & 78.75 & 85.00 & 91.25 \\
\hline
\end{tabular}

\section{B. The CSS's Financial Situation ${ }^{2}$}

7. The CSS's expenditure accounts for almost 9 percent of GDP, and is roughly half of Panama's total social expenditure (including expenditure on education). Transfer payments represent about 56 percent of current expenditure; the remaining 44 percent are operational expenditure for benefits in kind (e.g., services provided under the health program) and administrative costs. In 1998, the CSS's revenue was structured as follows: (1) 79 percent from contribution revenue; (2) 5 percent from public transfers to cover administrative costs; and (3) 16 percent from "other sources," mainly the earnings on its accumulated surplus. ${ }^{3}$

8. For 1999 , the CSS is projected to run a surplus of about US\$160 million. The financial balance is in surplus even excluding the government transfer. Under the IVM program a capital reserve of about US\$1.4 billion has been accumulated, and is deposited with the Banco Nacional de Panama (BNP).

${ }^{2}$ This section draws on a recent study conducted by the International Labor Organization (ILO) which evaluates the CSS's actuarial situation. It is henceforth referred to as "the ILO report".

${ }^{3}$ Employers' lack of compliance with their contribution obligations is a problem in Panama, as it is in most other Latin American countries. In many cases, companies not only neglect to pay the employer's share of the contribution, but also fail to remit the employee's share that they withhold from his or her pay-check. The stock of contribution arrears has reached US $\$ 50$ million ( 0.5 percent of GDP). This figure does not take into account the accumulated revenue loss from outright evasion or nonreporting. A law that will increase the penalties for employers' noncompliance is under preparation. 
9. Nevertheless, financial sustainability is not guaranteed over the medium term. The aging of Panama's population together with generous benefits will put pressure on the CSS's finances, in particular on its main program, the IVM pension scheme. The number of pensioners is projected to rise from 101,000 in 1998 to 292,000 in 2020. For every Panamanian who was 60 and older in 1995, there were almost eight working age Panamanians. By 2020 , there will be less than five.

Table 2. Panama: Demographic Development

\begin{tabular}{lcccccc}
\hline & 1995 & 2000 & 2005 & 2010 & 2015 & 2020 \\
\hline $\begin{array}{l}\text { Support ratio } \\
\text { (Population aged 15-59/Population } \\
\text { aged 60 and above) }\end{array}$ & 7.7 & 7.5 & 6.9 & 6.3 & 5.5 & 4.8 \\
$\begin{array}{l}\text { Total number of pensioners in IVM } \\
\text { Program (in thousands) }\end{array}$ & 101 & 120 & 154 & 191 & 238 & 292 \\
\hline
\end{tabular}

10. The reserves of the CSS are well below its legal reserve requirement. The IVM scheme is intended to operate as a partially funded pay-as-you-go scheme. The Organic Law, Article 34 and $34 a$, requires the CSS to maintain a level of capital reserves equal to the present value of all the pensions that have been granted under the IVM program as of the date the reserves are valued. The ILO report's analysis shows that this rule implies that reserves should be about 9 to 10 times the scheme's annual pension outlays. Given the IVM's annual outlays of almost US $\$ 360$ million, its legal reserve should therefore be around US $\$ 3.5$ billion. Comparing this figure to the actual capital reserve of US $\$ 1.4$ billion, results in a shortfall of about US $\$ 2.1$ billion. Even if it fulfilled its legal reserve obligation, the CS\$ would not be fully capitalized, and so would be actuarially unbalanced. The Organic Law's reserve definition only requires funding for the pensions currently being paid, however, it does not take into account the present value of the accumulated claims of those who currently contribute to the system. To account for them, the CSS would need a capital reserve of US $\$ 8.5$ billion. By this definition the shortfall would reach US $\$ 7.1$ billion.

11. Whether the reserve shortfall is calculated relative to the legally required reserve or this second and broader definition, it is clear that the system's finances have deteriorated. The legal reserve shortfall of US\$2.1 billion now represents over 150 percent of the IVM program's book value reserve (US $\$ 1.4$ billion). In 1984, the shottfall, calculated in the same way, amounted to 50 percent of the program's reserve. This means that over the past 15 years the CSS has never been able to fund its pension commitments to the level required by its Organic Law, and that the gap between its actual funding level and the level stipulated by the law has widened. 
12. Actuarial calculations aside, the fact that the revenue from contributions is not covering the CSS's current expenditure together with the projected decline in the support ratio are clear indicators of future financial pressures. A financial surplus is only achieved today because of the financial income from the system's capital reserves. By the year 2013, the ILO report predicts, the CSS's current revenue from both contributions and financial investments will no longer suffice to cover its expenditure. The CSS will then have to encroach on its reserves to pay its pension obligations. In the meantime, the widening gap between pensions and contributions will, other things unchanged, contribute to a growing public sector borrowing requirement.

\section{The Complementary Public Pension Scheme FCPS and its Conversion to SIACAP}

13. Until 1999, the FCPS functioned as a complementary scheme to the CSS with participation in it being obligatory for civil servants. Its benefit was designed to "top up" the benefit provided under the CSS plan to provide a 100 percent replacement of a civil servant's salary upon retirement. Already before the FCPS was found in 1975, there had been Special Laws (Leyes Especiales) in place since the 1930s, which provided very favorable treatment for certain groups of civil servants.

14. The FCPS was underfunded almost from its inception. It was supposed to operate as a pay-as-you-go system, but received a contribution of only 2 percent of a civil servant's salary. At the same time, given an average CSS salary replacement between 70 percent and 80 percent, the additional benefit payment required to lift a civil servant's pension to the promised 100 percent of the pensionable base could often be substantial. The burden on the FCPS was further increased by a backloaded pension formula, which created a strong incentive for strategic behavior: salary increases close to retirement would significantly raise a worker's pension at no direct cost for his manager's programmed budget. ${ }^{4}$ As a result, the scheme was clearly in the red in 1978 and never managed to balance its finances thereafter.

15. By 1994 the annual deficit of the FCPS had reached US\$34 million ( 0.5 percent of GDP)-a sizeable amount given the relatively small number of persons benefiting from the scheme. The scheme's contributions covered less than 40 percent of its expenditure. Its actuarial deficit was estimated to be near US\$870 million, implying that its cash-flow imbalance would increase further in the future. This situation did not only imply growing pressure on the budget, stemming from the transfers that were necessary to cover for unfunded benefit outlays. It was also inequitable, since the general taxpayer was helping to finance generous pensions to FCPS participants-comprising active contributors and retirees-who only constituted 6 percent of the total population.

16. The problems with the FCPS led to the decision to convert it into a voluntary, selffinanced, defined contribution, private pension capital scheme, the Sistema de Ahorros $y$

\footnotetext{
${ }^{4}$ The reference wage was based on the average of the five best salaries of the last 15 years of work.
} 
Capitalización de Pensiones de los Servidores Públicos (SLACAP). A reform agenda was worked out with assistance from the Inter-American Development Bank (IDB) and the World Bank, and the government officially embarked on the conversion process with a law passed in February 1997. The basic rationale for reform was the need to halt the continuous drain on the budget and solve the connected equity problem. However, the introduction of SIACAP was also expected to help increase Panama's private sector saving ratio and foster the development of the domestic capital market.

17. Recognition bonds of US\$397 million were issued to the new system to cover the value of the contributors' past contributions. ${ }^{5}$ These liabilities result from the contribution payments participants had made between 1975 and 1997. A simple summation of the contributions recorded in the system's books over this period yields a figure of US $\$ 225$ million. The net present value of these contributions, hence the implicit liability to be covered by recognition bonds, was calculated by using an interest rate of 5 percent. The current impact of this operation on the public finances is attenuated by the fact that recognition bonds are nonnegotiable and redeemable only at retirement. However, recurring debt service costs will commence immediately.

18. SIACAP's investments are subject to several legal restrictions. Only 10 percent of the capital can be invested abroad. Moreover, 30 percent to 50 percent must be invested in government guaranteed financial instruments. An administrative counsel for the SLACAP was appointed in 1998 and fund managers were contracted through public bidding and shouk commence their work soon.

19. The law allows several options for holders of redeemable recognition bonds. These include the purchase of a life annuity, full withdrawal of accumulated contributions upon retirement, a programmed withdrawal, or a combination of these. ${ }^{6}$ It is estimated that about 800 to 900 participants are already eligible to redeem their recognition bonds. It is not clear, however, whether these persons are aware of this right.

20. So far, about 60,000 active civil servants have joined SIACAP. Since the conversion to SIACAP affected every person who had contributed to the FCPS in the past, the SIACAP's books record a total of 234,000 persons. However, about 84,000 are former civil servants, including persons affected by the privatization of public enterprises who no longer work for the public sector. Of the remaining 150,000 active civil servants, about 90,000

\footnotetext{
${ }^{5}$ This value would be less than the future pension benefits promised to plan participants under the scheme, since the value of contributions plus interest would fall well short of the present expected value of contributors' pensions.

${ }^{6}$ Under a programmed withdrawal, the retiree and the insurance company agree on a stream of withdrawals over a given period of time. A similar option exists under the privatized Chilean system, and is quite popular.
} 
decided not to participate in the SIACAP, as it has now become a voluntary scheme. Those who joined SIACAP continue to contribute at the rate of 2 percent of their salary, as before under the FCPS.

21. Pension levels for civil servants will drop owing to SIACAP. The level of benefits it provides will depend on the value of contributions made and the rate of return they earn.

World Bank estimates, assuming a real rate of return of 3 percent to 4 percent, show that the current contribution rate of 2 percent would provide a life annuity of only about 8 percent to 10 percent of a civil servant's last salary. Thus, a civil servant whose CSS pension is below 90 percent-as is the case for everyone with less than 40 years of contribution paymentswill receive pensions whose combined replacement rate is below the 100 percent previously guaranteed by the FCPS.

\section{Strategies for Further Reform}

22. The conversion of the FCPS is a first step toward a comprehensive reform of the whole system. Although the CSS is not as yet in a state of crisis, its financial trend shows the need to reconsider existing minimum ages, pension formulas, benefit structures and the system's administrative efficiency. The system has not undergone any significant changes since its (partial) reform in 1992, when the pensionable age and contribution rates were increased to their current levels.

23. The statutory retirement age is low in Panama-particularly for women, even when compared with that of other countries with similar demographic characteristics and life expectancies. The five-year difference with respect to the retirement age of men is large, notwithstanding the common practice in public pension schemes of allowing women to retire at an earlier age than men. ${ }^{8}$ One possible refonm could be to raise the retirement age to 60 for women and 63 for men in a first step, and possibly further to 62 and 65 respectively, provided some difference between men's and women's retirement age is to be maintained. Such retirement ages are common in public pension schemes around the world, and they still only partly compensate for the general increase in longevity over the past decades.

24. Further, the link between contributions and benefits should be strengthened. The increase in the pension that results from an additional year of contribution is low: 1/4 percent. This can be seen as the consequence of the relatively high minimum pension of 60 percent of the reference salary for plan participants who have only 15 years of contributions, since a

\footnotetext{
${ }^{7}$ There have been problems in convincing some groups of public servants to enroll into the SIACAP scheme. For example, some teachers' unions recommend to their members that they not join the scheme.

${ }^{8}$ As women on the average also live longer than men, this practice is definitely not justifiable on an actuarial basis. The European Union has obliged its member states to set unified statutory retirement ages for men and women as to avoid discrimination.
} 
higher accrual rate would entail even higher replacement ratios. The present benefit formula creates an incentive for plan participants to keep their contribution period to a minimum, since they can still receive a sizeable pension without many years of contributions. Lowering the minimum replacement rate below 60 percent and increasing the accrual rate would strengthen the contribution link, and thereby reduce the adverse incentive effect the present benefit formula creates. Additional years of contributions would increase the value of the pension by more than they do now.

25. The IVM's high replacement levels should gradually be reduced. As noted above, old-age pensions reach a level of almost 90 percent of the average Panamanian wage. Consequently, a reduction of these benefits would be possible without jeopardizing the CSS's objective of providing basic income security for the elderly. Lower pension levels could make an important contribution to fiscal consolidation, and would also be related more appropriately to the IVM's relatively low contribution rate: to reach replacement levels of up to 90 percent of the seven highest annual salaries by only contributing 9.5 percent of all salaries is not possible in the mature public pension schemes of other countries.

26. As an alternative to tackling expenditure, the ILO report outlines a strategy to achieve actuarial balance from the revenue side. It recommends augmenting the system's capital reserves and setting out a time schedule for the contribution rate increases necessary in order to meet these capitalization requirements. However, given the relative generosity of benefits, and the need to keep labor costs low, payroll tax increases are probably not advisable. Reforms should instead emphasize changes to the benefit formula and the minimum age.

\footnotetext{
${ }^{9}$ Many European schemes offer replacement levels close to those of the Panamanian CSS, but they also require much higher contributions. In the German public pension scheme, for example, replacement rates may reach $70-80$ percent, but they are funded by a contribution rate of 20 percent; i.e., more than double the IVM's 9.5 percent rate.
} 
Table 3. Panama: National Accounts by Productive Activity

\begin{tabular}{|c|c|c|c|c|c|}
\hline & 1994 & 1995 & 1996 & 1997 & 1998 \\
\hline \multicolumn{6}{|c|}{ (In millions of belboas at 1982 prices) } \\
\hline Gross domestic product at market prices & $6,091.3$ & $6,198.0$ & $6,372.2$ & $6,657,4$ & $6,931.8$ \\
\hline Primary activity & 502.4 & 517.8 & 522.2 & 528.5 & 546.1 \\
\hline Agriculture & 493.3 & 509.2 & 515.0 & 514.9 & 535.6 \\
\hline Mining & 9.1 & 8.6 & 7.2 & 13.6 & 10.5 \\
\hline Secondary activity & 863.2 & 874.6 & 856.2 & 908.6 & 945.9 \\
\hline Manufacturing & 614.6 & $6 ! 5.8$ & 608.1 & 646.8 & 671.0 \\
\hline Construction & 248.6 & 258.8 & 248.1 & 261.8 & 274.9 \\
\hline Services & $4,710,1$ & $4,784.2$ & $4,984.2$ & $5,225.9$ & $5,434.3$ \\
\hline Public utilities & 255.0 & 259.9 & 305.9 & 308.2 & 292.8 \\
\hline \multicolumn{6}{|l|}{ Commerce, restaurants, } \\
\hline and hotels & $1,289.8$ & $1,280.4$ & 1.267 .8 & $1,381,2$ & $1,424.9$ \\
\hline Colon Free Zone wholesale & 533.6 & 516.6 & 490.2 & 570.5 & 571.5 \\
\hline Restaurants and hotels & 90.3 & 95.0 & 100.9 & 107.7 & $1\} 3.4$ \\
\hline Other & 665.9 & 668.8 & 676.7 & 703.0 & 740.0 \\
\hline Transport and communications & 655.8 & 761.3 & 780.1 & 824.9 & 876.1 \\
\hline Panama Canal Commission & 362.4 & 419.4 & 429.9 & 421.9 & 426.1 \\
\hline Dther transport and cotmm. & 333.4 & 341.9 & 350.2 & 4030 & 450.0 \\
\hline Financial intermediation & 675.0 & 641.8 & 725.4 & 729.2 & 787.8 \\
\hline Housing & 821.1 & 841.4 & 879.2 & 908.8 & 948.4 \\
\hline Public adrninistration & 632.0 & 653.5 & 665.3 & 690.6 & 711.3 \\
\hline Other services & 341.4 & 345.9 & 360.5 & 383.0 & 393.0 \\
\hline Plus: import taxes 2f & 245.2 & 249.8 & 256.5 & 270.4 & 293.5 \\
\hline Less: tmputed banking services & 229.6 & 228.4 & 246.9 & 276.0 & 288.0 \\
\hline \multicolumn{6}{|c|}{ (Percent change) } \\
\hline Gross domestic product at 1982 market prices & 2.9 & 1.8 & 2.8 & 4.5 & 4.1 \\
\hline Primary activity & 2.8 & 3.1 & 0.8 & 1.2 & 3.3 \\
\hline Agriculture & 2.7 & 3.2 & 1.1 & 0.0 & 4.0 \\
\hline Mining & 9.6 & -5.5 & -16.3 & 88.9 & -22.8 \\
\hline Secondary activity & 3.7 & 1.3 & -2.1 & 6.1 & 4.1 \\
\hline Manufactaring & 4.3 & 0.2 & -1.3 & 6.4 & 3.7 \\
\hline Construction & 2.2 & 4.1 & -4.1 & 5.5 & 5.0 \\
\hline Services & 4.5 & 1.6 & 4.2 & 4.8 & 4.0 \\
\hline Public utilities & 6.3 & 1.9 & 17.7 & 0.8 & -5.0 \\
\hline \multicolumn{6}{|l|}{ Commeree, restararants, } \\
\hline and hotels & 4.9 & -0.7 & -1.0 & 8.9 & 3.2 \\
\hline Colon Free Zone wholesale & 9.3 & -3.2 & -5.1 & 6.4 & 0.2 \\
\hline Restaurants and hotels & 5.7 & 5.2 & 6.2 & 6.7 & 5.3 \\
\hline Other & $\$ .5$ & 0.4 & 12 & 3.9 & 5.3 \\
\hline Transport and communications & 4.4 & 9.4 & 2.5 & 5.7 & 6.2 \\
\hline Panama Canal Commission & 3.0 & 15.7 & 2.5 & -1.9 & 1.0 \\
\hline Other transpott and comm. & 6.1 & 2.5 & 2.4 & 15.1 & 11.7 \\
\hline
\end{tabular}


Table 3. Panama: National Accounts by Productive Activity (Concluded)

\begin{tabular}{|c|c|c|c|c|c|}
\hline & 1994 & 1995 & 1996 & 1997 & 1998 \\
\hline Financial intermediation & 4.3 & 4.9 & 13.0 & 0.5 & 8.0 \\
\hline Housing & 4.4 & 2.5 & 4.5 & 3.4 & 4.4 \\
\hline Publis administration & 3.3 & 3.4 & 5.8 & 3.8 & 3.0 \\
\hline Other services & 4.9 & 1.3 & 4.2 & 6.2 & 2.6 \\
\hline Plus: import taxes $2 /$ & 3.9 & 1.9 & 2.7 & 5.4 & 8.5 \\
\hline Less: imputed banking services & 62.3 & -0.5 & 8.1 & 11.8 & 4.3 \\
\hline \multicolumn{6}{|c|}{ (Percent distribution) } \\
\hline Gross domestic product at 1982 market prices & 100.0 & 100.0 & 100.0 & 100.0 & 100.0 \\
\hline Primary activity & 8.2 & 8.4 & 8.2 & 7.9 & 7.9 \\
\hline Agrieulture & 8.1 & 8.2 & 8.1 & 7.7 & 7.7 \\
\hline Mining & 0.1 & 0.1 & 0.1 & 0.2 & 02 \\
\hline Secondary activity & 14,2 & 14.1 & 13.4 & 13.6 & 13.6 \\
\hline Manufacturing & 10.1 & 9.9 & 9.5 & 9.7 & 9.7 \\
\hline Construction & 4.1 & 4.2 & 3.9 & 3.9 & 4.0 \\
\hline Services & 77.3 & 77.2 & 78.2 & 78.5 & 78.4 \\
\hline Public utilities & 4.2 & 4,2 & 4.8 & 4.6 & 4.2 \\
\hline \multicolumn{6}{|l|}{ Commerce, restaurants, } \\
\hline and hotels & 21.2 & 20.7 & 19.9 & 20.7 & 20.6 \\
\hline Colon Free Zone wholesale & 8.8 & 8.3 & 7.7 & 8.6 & 8.2 \\
\hline Restaurants and hotels & 1.5 & 1.5 & 1.6 & 1.6 & 1.6 \\
\hline Other & 10.9 & 10.8 & 10.6 & 10.6 & 10.7 \\
\hline Transport and communications & 11.4 & 12.3 & 12.2 & 12.4 & 12.6 \\
\hline Panama Canal Commission & 5.9 & 6.8 & 6.7 & 6.3 & 6.1 \\
\hline Other transport and comm. & 5.5 & 5.5 & 5.5 & 6.1 & 6.5 \\
\hline Financial intermediation & 11.1 & 10.4 & 11.4 & 11.0 & 11.4 \\
\hline Housing & 13.5 & 13.6 & 13.8 & 13.7 & 13.7 \\
\hline Public administration & 10.4 & 10.5 & 10.4 & 10.4 & 10.3 \\
\hline Other services & 5.6 & 5.6 & 5.7 & 5.8 & 5.7 \\
\hline Plus: import taxes $2 /$ & 4.0 & 4.0 & 4.0 & 4.1 & 42 \\
\hline Less: imputed barking services & 3.8 & 3.7 & 3.9 & 4.1 & 4.2 \\
\hline
\end{tabular}

Sources: Office of the Comptroller General; and Fund staff estimates.

V The national accounts for $1980-94$ were revised and published in December 1995 . The base year for the constant piree data was updated to 1982 from 1970 and methodological improvements were impiemented.

2f Includes the sales tax: "impuesto a la transferencia de bienes muebles." 
Table 4. Panama: National Accounts by Use

\begin{tabular}{|c|c|c|c|c|c|}
\hline & 1994 & 1995 & 1996 & 1997 & 1998 \\
\hline \multicolumn{6}{|c|}{ (In millions of balboas at purrent prices) } \\
\hline Total domestic deminand & $7,644.6$ & $8,063.4$ & $8,270.6$ & $9,000.0$ & $9,990.0$ \\
\hline Consumption & 5,5710 & $5,669.2$ & $5,783.2$ & $6,308.9$ & $6,858.7$ \\
\hline Public & 1,0073 & $1,029.7$ & $1,0.55 .4$ & $1,176.2$ & $1,241.9$ \\
\hline Privale & $4,563.7$ & $4,639.5$ & $4,727.8$ & $5,132.7$ & $5,616.8$ \\
\hline Gross domestic irvestment & $2,073.6$ & $2,394.2$ & $2,487.4$ & $2,691.1$ & $3,131.3$ \\
\hline Publio & 266.7 & 272.0 & 313.4 & 374.6 & 583.3 \\
\hline Private I/ & $1,806.9$ & $2,122.2$ & $2,174.0$ & $2,316.5$ & $2,548.0$ \\
\hline Balanoe of trade (goots and nonfactor services) & 89.3 & -157.3 & -119.5 & -342.5 & -846.2 \\
\hline Balance excluding the Colon Free Zone & -824.7 & -764.8 & -920.4 & $-1,156.2$ & $-1,280.8$ \\
\hline Exports of goods and nonfactor services & $7,448.5$ & $7,610.3$ & $7,381.5$ & $8,303.8$ & $8,022.6$ \\
\hline Exports excluting the Colon Free Zone & $2,069.0$ & $2,264.0$ & $2,299.0$ & $2,493.0$ & $2,562.0$ \\
\hline Imports of goods and nonfactor services & $7,359.2$ & $7,767.6$ & $7,50 \mathrm{~L} .0$ & $8,646.3$ & $8,868.8$ \\
\hline Imports excluding the Colon Free Zone & $2,893.7$ & $3,028.8$ & $3,219.4$ & $3,649.2$ & $3,842.8$ \\
\hline Gross domestic product at current market prices & $7,733.9$ & $7,906.1$ & $8,15 \mathrm{~L} .1$ & $8,657.5$ & $9,143.8$ \\
\hline \multicolumn{6}{|c|}{ (In pereent of GDP) } \\
\hline Total domaestic demand & 98.9 & 101.9 & 101.4 & 104.0 & 1093 \\
\hline Consumption & 72.0 & 71.7 & 70.9 & 72.9 & 75.0 \\
\hline Public & 13.0 & 13.0 & 12.9 & 13.6 & 13.6 \\
\hline Private & 59.0 & 58.7 & 58.0 & 59.3 & 61.4 \\
\hline Grass domestic investment & 26.8 & 30.2 & 30.5 & 31.1 & 34.3 \\
\hline Public & 3.4 & 3.4 & 3.8 & 4.3 & 6.4 \\
\hline Private $\mathbf{l}$ & 23.4 & 26.8 & 26.7 & 26.8 & 27.9 \\
\hline \multicolumn{6}{|c|}{ (In percert of GDP) } \\
\hline Balano of trade (goods and nonfactor services) & 1.2 & -2.0 & .1 .5 & -4.0 & -9.3 \\
\hline Balance exciading the Colon Free Zore & -10.7 & -9.7 & -11.3 & -13.4 & -14.0 \\
\hline Exports of goods ard nonfactor services & 96.3 & 96.3 & 90.6 & 95.9 & 87.7 \\
\hline Exports exctuding the Colon Free Zone & 26.8 & 28.6 & 28.2 & 28.8 & 28.0 \\
\hline Imports of goods and nonfactor services & 95.2 & 98.2 & 92.0 & 99.9 & 97.0 \\
\hline Inports excluding the Colon Free Zone & 37,4 & 38.3 & 39.5 & 42.2 & 42.0 \\
\hline Grose domestic product at current market prices & 100.0 & 100,0 & 100.0 & 100.0 & 100.0 \\
\hline \multicolumn{6}{|c|}{ (Percentage change) } \\
\hline Total domestic demand & 5.2 & 5.5 & 2.6 & $\mathbf{8 . 8}$ & 11.0 \\
\hline Consumption & 2.6 & 1.8 & 2.0 & 9.1 & 8.7 \\
\hline Public & 6.6 & 2.2 & 2.5 & 11.4 & 5.6 \\
\hline Private & 1.7 & 1.7 & 1.9 & 8.6 & 9.4 \\
\hline Gross domestic investment & 13.0 & 15.5 & 3.9 & 8.2 & 16.4 \\
\hline Publis & -7.7 & 2.0 & 15.2 & 19.5 & 55.7 \\
\hline Private $1 /$ & 16.8 & 17.4 & 2.4 & 6.6 & 10,0 \\
\hline Exports of goods and nonfactor services & 10.9 & 2.2 & -3.0 & 12.5 & -3.4 \\
\hline Exports excluding the Colon Free Zone & 3.5 & 9.4 & 1.5 & 8.4 & 2.8 \\
\hline Imports of goods and nonfactor services & 9.4 & 5.5 & -3.4 & 15.3 & 2.6 \\
\hline Inports excluding the Colon Free Zone & 6.4 & 4.7 & 6.3 & 13.4 & 3.3 \\
\hline Gross domestic prodiuct at current market prices & 6.6 & 2.2 & 3.1 & 6.2 & 5,6 \\
\hline
\end{tabular}

Sources: Office of the Cormptroller General; and Fund staff estimates.

I/ Includes changes in inventories. 
Table 5. Panama: Savings and Investment

\begin{tabular}{|c|c|c|c|c|c|}
\hline & 1994 & 1995 & 1996 & 1997 & 1998 \\
\hline \multicolumn{6}{|c|}{ (In millions of balboas) } \\
\hline Gross domestic investment & $2,073.6$ & $2,394.2$ & $2,487,4$ & $2,691.1$ & $\mathbf{3 , 1 3 1 . 3}$ \\
\hline Fixed capital formation (gross) & $1,828.8$ & $2,057.9$ & $2,059.2$ & $2,295.0$ & $2,708.3$ \\
\hline Public sector & 266.7 & 272.0 & 313.4 & 374.6 & 583.3 \\
\hline Private sector & $1,562.1$ & $1,785.9$ & $1,745.8$ & $1,920.4$ & $2,125.0$ \\
\hline Changes in invertories & 244.8 & 336.3 & 428.2 & 396.1 & 423.0 \\
\hline Gross national savings & $2,057.8$ & $2,025.1$ & $2,185.5$ & $2,087.6$ & $1,919.6$ \\
\hline Public sector savings & 265.9 & 262.8 & 323.9 & 260.0 & 224.0 \\
\hline Private sector savings & $1,791.9$ & $1,762.3$ & $1,861.6$ & $1,827.6$ & $1,695.6$ \\
\hline Fortign savings & -15.8 & 369.1 & 301.9 & 603.5 & 1211.7 \\
\hline \multicolumn{6}{|c|}{ (In percent of GDP) } \\
\hline Gross domestic investment & 26.8 & 30.2 & 30.5 & 31.1 & 34.3 \\
\hline Fixed capital formation (gross) & 23.6 & 26.0 & 25.3 & 26.5 & 29.6 \\
\hline Public sector & 3.4 & 3.4 & 3.8 & 4.3 & 6.4 \\
\hline Private sector & 20.2 & 22.6 & 21.4 & 22.2 & 23.2 \\
\hline Changes in inventories & 3.2 & 4.3 & 5.3 & 4.6 & 4.6 \\
\hline Gross national savings & 26.6 & 25.6 & 26.8 & 24.1 & 21.0 \\
\hline Public sector savings & 3.4 & 3.3 & 4.0 & 3.0 & 2.4 \\
\hline Private sector savings & 23.2 & 22.3 & 22.8 & 21.1 & 18.5 \\
\hline Foreign savings & -0.2 & 4.7 & 3.7 & 6.9 & 13.3 \\
\hline
\end{tabular}

Sources: Office of the Comptroller General; and Fund staff estimates. 
Table 6. Panama: Agricultural Production

\begin{tabular}{|c|c|c|c|c|c|}
\hline & 1994 & 1995 & 1996 & 1997 & 1998 \\
\hline \multicolumn{6}{|c|}{ (In millions of balboas at 1982 prices) } \\
\hline Total & 493.3 & 509.2 & 515.0 & 514.9 & 545.1 \\
\hline Crops & 269.0 & 262.9 & 261.6 & 242.3 & 244.4 \\
\hline Rice & 32.3 & 33.2 & 36.0 & 25.0 & 40.0 \\
\hline Com & 17.3 & 17.6 & 18.2 & 13.7 & 16.5 \\
\hline Bananas & 136.1 & 126.6 & 117.6 & $\mathbf{1} 12.4$ & 86.0 \\
\hline Sugarcane & 19.3 & 18.2 & 19.6 & 21.7 & 23.9 \\
\hline Coffee & 13.2 & 15.0 & 15.5 & 14.6 & 15.9 \\
\hline Other & 50.8 & 52.3 & 54.7 & 54.9 & 62.1 \\
\hline Livestock & 157.3 & 159.3 & 167.5 & 174.2 & 188.0 \\
\hline Forestry & 14.6 & 12.8 & 12.0 & 12.7 & 12.7 \\
\hline Fish & 52.4 & 74.2 & 73.9 & 85.7 & 100.0 \\
\hline \multicolumn{6}{|c|}{ (Annual percent change) } \\
\hline Total & 2.7 & 3.2 & 1.1 & 0.0 & 5.9 \\
\hline Crops & 3.7 & -2.3 & -0.5 & -7.4 & 0.9 \\
\hline Livestock & 1.5 & 1.3 & 5.1 & 4.0 & 7.9 \\
\hline Forestry & -0.7 & -12.3 & -6.3 & 5.8 & 0.0 \\
\hline Fish & 2.1 & 41.6 & -0.4 & 16.0 & 16.7 \\
\hline
\end{tabular}

Sources: Office of the Comptroller General; and Fund staff estimates. 
Table 7. Panama: Value-Addied in Manufacturing

\begin{tabular}{|c|c|c|c|c|c|}
\hline & 1994 & 1995 & 1996 & 1997 & 1998 \\
\hline \multicolumn{6}{|c|}{ (in millions of balboas at 1982 prices) } \\
\hline Total & 614.6 & 615.8 & 608.1 & 646.8 & 673.4 \\
\hline Food & 237.1 & 245.5 & 246.4 & 265.5 & 295.2 \\
\hline Beverages & 53.7 & 52.7 & 53.3 & 58.4 & 63.2 \\
\hline Tobacco & 27.8 & 27.2 & 29.2 & 28.6 & 8.2 \\
\hline Petroleum products & 15,1 & 10.5 & 17.3 & 17.5 & 19.3 \\
\hline Textiles and garments & 38.1 & 34.4 & 30.3 & 27.8 & 25.8 \\
\hline Leather products and footwear & 11.6 & 13.0 & 10.9 & 9.8 & 8.7 \\
\hline Wood and furniture & 31.4 & 27.6 & 28.4 & 31.1 & 35.8 \\
\hline Paper products & 29.9 & 30.6 & 27.8 & 25.9 & 23.3 \\
\hline Printing and publishing & 15.2 & 16.2 & 12.0 & 11.9 & 16.8 \\
\hline Chemicals, rubber and plastic products & 57.6 & 59.3 & 55.2 & 57.3 & 56.8 \\
\hline Glass and other minerat products & 49,9 & 45.2 & 41.3 & 46.3 & 52.9 \\
\hline Basic metal industries & 9.8 & 10.9 & 13.7 & 18.0 & 20.4 \\
\hline \multicolumn{6}{|l|}{ Metal products, machinery, and } \\
\hline equipment & 21.5 & 22.7 & 22.2 & 26.0 & 28.0 \\
\hline Other & 15.9 & 20.0 & 20.1 & 22.7 & 19.0 \\
\hline \multicolumn{6}{|c|}{ (Annual percent change) } \\
\hline Total & 4.3 & 0.2 & -1.3 & 6.4 & 4.1 \\
\hline Petroleum products & -28.4 & 30.5 & 64.8 & 1.2 & 10.3 \\
\hline Total (excluding petroleum products) & 5.5 & 1.0 & -2.4 & 6.5 & 3.9 \\
\hline Food, beverages, and tobacco & 5.7 & 2.1 & 1.1 & 7.2 & 4.0 \\
\hline Other products & 5.2 & -0.4 & -6.4 & 5.7 & 3.9 \\
\hline
\end{tabular}

Sources: Office of the Comptroller General; and Fund staff estimates. 
Table 8. Panama: Electricity Generation and Consumption

(In thousands of gigawatt hours) $1 /$

\begin{tabular}{lccccc}
\hline & 1994 & 1995 & 1996 & 1997 & 1998 \\
\hline Net generation & $\mathbf{3 . 3 6}$ & $\mathbf{3 . 5 2}$ & $\mathbf{3 . 8 2}$ & $\mathbf{4 . 0 5}$ & $\mathbf{4 . 1 8}$ \\
Hydro & 2.39 & 2.42 & 3.00 & $\mathbf{2 . 9 0}$ & 2.14 \\
Themal & 0.97 & 1.10 & 0.82 & $\mathbf{1 . 1 5}$ & 2.04 \\
& & & & & \\
Total consumption & $\mathbf{2 . 6 7}$ & $\mathbf{2 . 8 7}$ & $\mathbf{2 . 9 8}$ & $\mathbf{3 . 3 0}$ & $\mathbf{3 . 4 1}$ \\
Residential & 0.79 & 0.85 & 0.86 & 0.94 & 1.00 \\
Commercial & 0.91 & 1.00 & 1.04 & 1.18 & 1.34 \\
Industrial & 0.43 & 0.46 & 0.47 & 0.47 & 0.49 \\
Government & 0.47 & 0.53 & 0.55 & 0.57 & 0.54 \\
Petroterminal & 0.01 & 0.01 & 0.00 & 0.00 & 0.00 \\
Other & 0.06 & 0.02 & 0.06 & 0.14 & 0.04 \\
& & & & & \\
\hline
\end{tabular}

Sources: Hydraulic Resources and Electricity Institute (IRHE); and Fund staff estimates.

I/ A gigawatt hour is equal to one billion watts being generated or consumed for one hour. 
Table 9. Panama: Private Sector Construction Permits, District of Panama

(In thousands of square meters)

\begin{tabular}{lrrrrr}
\hline & 1994 & 1995 & 1996 & 1997 & 1998 \\
\hline Total & & & & & \\
Residential & 912.4 & 827.9 & 925.3 & 795.6 & 535.5 \\
Commercial & 550.4 & 505.8 & 430.0 & 407.7 & 283.4 \\
Industrial & 299.7 & 227.0 & 438.9 & 360.3 & 222.5 \\
Other & 14.8 & 14.4 & 26.8 & 3.8 & 3.5 \\
& 47.6 & 30.7 & 29.6 & 23.8 & 26.1 \\
\hline
\end{tabular}

Sources: Office of the Comptroller General; and Fund staff estimates. 
Table 10. Panama: Domestic Sales of Petroleum

\begin{tabular}{lrrrrr}
\hline & 1994 & 1995 & 1996 & 1997 & 1998 \\
& & & & & \\
& (In millions of balboas) & & & \\
& & & & & \\
& & & & & \\
Domestic sales & 200.0 & 222.5 & 255.8 & 309.1 & 275.2 \\
Gasoline & 59.9 & 69.0 & 80.5 & 87.7 & 75.3 \\
Diesel & 88.5 & 93.7 & 103.8 & 126.9 & 120.5 \\
Fuel oil & 27.3 & 34.3 & 39.8 & 35.6 & 34.9 \\
Kerosene & 2.2 & 2.1 & 2.0 & 3.1 & 2.9 \\
LP gas & 17.3 & 19.4 & 22.1 & 33.1 & 21.9 \\
Other products 1/ & 4.8 & 4.0 & 7.6 & 22.7 & 19.7 \\
& & & & & \\
& (In millions of barrels) & & & \\
Domestic sales & & & & & \\
Gasoline & 9.0 & 9.7 & 9.8 & 10.8 & 12.8 \\
Diesel & 2.4 & 2.6 & 2.7 & 2.9 & 3.2 \\
Fuel oil & 3.7 & 4.1 & 3.6 & 4.3 & 5.3 \\
Kerosene & 1.7 & 1.8 & 1.9 & 1.7 & 2.2 \\
LP gas & 0.1 & 0.1 & 0.1 & 0.1 & 0.1 \\
Other products 1/ & 0.9 & 0.9 & 1.0 & 1.0 & 1.1 \\
& 0.2 & 0.2 & 0.5 & 0.8 & 0.9 \\
\hline
\end{tabular}

Sources: Ministry of Commerce and Industry; and Fund staff estimates.

1/ Includes marine diesel. 
Table 11. Panama: Labor Force Statistics

\begin{tabular}{|c|c|c|c|c|c|c|}
\hline & 1994 & 1995 & 1996 & 1997 & 1998 & $19991 /$ \\
\hline \multicolumn{7}{|c|}{ (In thousands of persons) } \\
\hline Total population & $2,582.6$ & $2,631.0$ & $2,674.5$ & $2,718.7$ & $2,764.9$ & $2,855.7$ \\
\hline Working age population & $1,594.7$ & $1,632.4$ & $1,670.2$ & $1,706.8$ & $1,742.9$ & $1,779.1$ \\
\hline Total labor force $2 /$ & 967.3 & $1,007.9$ & $1,012.1$ & $1,049,4$ & $1,083,6$ & $1,089.0$ \\
\hline Employment by activity & 831.8 & 866.7 & 867.2 & 909.1 & 936.5 & 962.3 \\
\hline Agriculture and mining & 167.9 & 181.9 & 176.0 & 171.6 & 167.3 & 169.9 \\
\hline Manufachuring & 89.5 & 92.6 & 94.0 & 96.2 & 90.6 & 92.6 \\
\hline Public utilities & 9.3 & 8.8 & 9.1 & 9.1 & 9.4 & 7.3 \\
\hline Construction & 52.5 & 53.6 & 54.9 & 59.6 & 67.9 & 73.8 \\
\hline Commerce & 146.1 & E52.1 & 153.0 & 168.1 & 177.5 & 182.4 \\
\hline Banking & 19.4 & 19.7 & 22.1 & 22.8 & 24.4 & 24.3 \\
\hline Transportation and communic & 60.7 & 63.4 & 59.0 & 62.1 & 63.7 & 73.0 \\
\hline Other services & 286.4 & 294.6 & 299.1 & 319.6 & 335.7 & 339.0 \\
\hline Unemployment & 135.5 & 141.3 & 144.9 & 140.3 & 147.1 & 126.7 \\
\hline Unemployment rate & 16.0 & 14.0 & 14.3 & 13.4 & 13.6 & 11.6 \\
\hline \multicolumn{7}{|l|}{$\begin{array}{l}\text { Memorandum items: } \\
\text { Number of employed by: }\end{array}$} \\
\hline Employment by sector & 831.9 & 866.7 & 867.2 & 909.1 & 936.5 & 962.3 \\
\hline Public sector & 163.3 & 161.4 & 160.2 & 161.8 & 167.2 & 156.1 \\
\hline Private business & 340.4 & 354.3 & 359.4 & 372.7 & 394.3 & 424.1 \\
\hline Self-employed & 232.8 & 247.0 & 250.3 & 275.9 & 282.6 & 296.1 \\
\hline Other & 95.4 & 103.9 & 97.3 & 98.7 & 92.4 & 85.9 \\
\hline \multicolumn{7}{|c|}{ (Annual percent change) } \\
\hline Total population & 1.9 & 1.9 & 1.7 & 1.7 & 1.7 & 1.7 \\
\hline Working age population & 2.4 & 2.4 & 2.3 & 2.2 & 2.1 & 2.1 \\
\hline Total labor force & 2.9 & 4.2 & 0.4 & 3.7 & 3.3 & 0.5 \\
\hline Employed & 2.0 & 4.2 & 0.1 & 4.8 & 3.0 & 2.8 \\
\hline Unemployed & 8.7 & 4.3 & 2.5 & -3.2 & 4.9 & -13.9 \\
\hline \multicolumn{7}{|c|}{ (In percent) } \\
\hline Labor force participation rate $3 /$ & 60.7 & 61.7 & 60.6 & 61.5 & 62.2 & 61.2 \\
\hline Employment rate $4 /$ & 52,2 & 53.1 & 51.9 & 53.3 & 53.7 & 54.1 \\
\hline $\begin{array}{l}\text { Unemployment rate } \\
\text { of which: }\end{array}$ & 14.0 & 14.0 & 14.3 & 13.4 & 13.6 & 11.6 \\
\hline Metropolitan areas $5 /$ & & 16.6 & 16.9 & 15.4 & 16.0 & 13.8 \\
\hline
\end{tabular}

Sources: Ministry of Labor; Office of the Comptroller Generai; and Fund staff estimates.

1/ As of August 1999 household survey.

2/ Excludes indigenous population, caral area and collective housing, but includes employees in the formal and informal sectors, employers, and the self employed.

3/ Total labor force as a percent of the working-age population.

4/ Employed labor force as a percent of the working-age population.

5/ Comprises the cities of Panama and Colon. 
Table 12. Panama: Average Prices Received by Farmers

\begin{tabular}{|c|c|c|c|c|c|}
\hline & 1994 & 1995 & 1996 & 1997 & 1998 \\
\hline \multicolumn{6}{|c|}{ (In balboas per unit) } \\
\hline Rice $(100 \mathrm{lb}$.) $1 /$ & 12.56 & 12.87 & 11.91 & 12.32 & 12.68 \\
\hline Corn $(100 \mathrm{lb})$. & 11.67 & 11.86 & 12,34 & 12.26 & 12.61 \\
\hline Beans (100 lb.) $2 /$ & 33.12 & 35.13 & 32.77 & 38.53 & 35.43 \\
\hline Potatoes $(100 \mathrm{Ib})$. & 26.29 & 23.40 & 24,72 & 19.30 & 21.81 \\
\hline Bananas stalk & 2,68 & 2.17 & 2.61 & 2.76 & 2.82 \\
\hline Chicken (live, lb.) & 1.23 & 1.24 & 1.21 & 1.23 & 1.25 \\
\hline Milk, fresh (bottle) & 0.30 & 0.29 & 0.27 & 0.28 & 0.28 \\
\hline \multicolumn{6}{|c|}{ (In 1987 prices) 31} \\
\hline Rice $(100 \mathrm{lb}) 1 /$. & 11.07 & 11.37 & 10.73 & 11.03 & 11.18 \\
\hline Corn (100 lb.) & 10.29 & 10.48 & 11.11 & 10.97 & 11.12 \\
\hline Beans (100 Ib.) $2 /$ & 29.19 & 31.04 & 29.52 & 34.49 & 31.24 \\
\hline Potatoes $(100 \mathrm{lb})$. & 23.17 & 20.68 & 22,27 & 17,27 & 19.23 \\
\hline Bananas stalk & 2.36 & 1.92 & 2.35 & 2.47 & 2.49 \\
\hline Chicken (live, lb.) & 1.08 & 1.10 & 1.09 & 1.10 & 1.10 \\
\hline Milk, fresh (bottle) & 0.26 & 0,26 & 0.24 & 0.25 & 0.25 \\
\hline
\end{tabular}

Sources: Office of the Comptroller General; and Fund staff estimates.

1/ First grade.

2/ Colorado or Chiricano.

3/ Deflated by the agricultural wholesale price index. 
Table 13. Panama: Selected Price Indices 1/

(Annual percent change)

\begin{tabular}{|c|c|c|c|c|c|c|}
\hline & 1994 & 1995 & 1996 & 1997 & 1998 & 1999 \\
\hline \multicolumn{7}{|c|}{ (Period average) } \\
\hline Consumer price index & 1.3 & 0.9 & 1.3 & 1.2 & 0.6 & 1,4 \\
\hline Wholesale price index & 2.0 & 3.0 & 2.1 & -2.2 & -3.9 & $\ldots$ \\
\hline Imports & 1.9 & 3.9 & 3.8 & -4.1 & -5.7 & $\ldots$ \\
\hline Industrial products & 2.2 & 2.7 & 1.3 & -0.9 & -3.3 & $\ldots$ \\
\hline Agricultural products & 2.5 & -0.2 & -1.9 & 0.6 & 1.5 & $\ldots$ \\
\hline GDP deflator & 3.7 & 0.5 & 0.3 & 1.7 & 1.4 & $\cdots$ \\
\hline \multicolumn{7}{|c|}{ (End of period) } \\
\hline Consumer price index & 1.3 & 0.8 & 2.3 & -0.5 & 1.4 & 1.5 \\
\hline Wholesale price index & 4.4 & 1.8 & 3.5 & -3.9 & -3.9 & $\ldots$ \\
\hline Imports & 5.1 & 2.8 & 6.0 & -6.3 & -5.5 & $\ldots$ \\
\hline Industrial products & 4.4 & 1.5 & 2.2 & -2.5 & -3.4 & $\ldots$ \\
\hline Agricultural products & 2.1 & -1.0 & $-0,9$ & 0.8 & 1.5 & $\ldots$ \\
\hline
\end{tabular}

Sources: Office of the Comptroller General; and Fund staff estimates.

1/ The consumer and wholesale price indices use 1987 as the reference period. 
Table 14. Panama: Consumer Price Index

$(1987=100)$

\begin{tabular}{|c|c|c|c|c|c|}
\hline & Total & Food & Clothing & Housing & Miscellaneous \\
\hline \multicolumn{6}{|c|}{ (In percent) } \\
\hline Weights: & 100.0 & 34.9 & 5.1 & 21.0 & 39.0 \\
\hline \multicolumn{6}{|c|}{ (Period average) } \\
\hline 1994 & 106.5 & 110.0 & 111.5 & 103.6 & 104.1 \\
\hline 1995 & 107.5 & 110.6 & $1 \pm 0.0$ & 105.4 & 105.5 \\
\hline 1996 & 108.9 & 111.4 & 109.0 & 108.2 & 106.9 \\
\hline 1997 & 110.2 & 112.2 & 106.8 & 110.5 & 108.7 \\
\hline 1998 & 110.9 & 112.6 & 108.2 & 111.7 & 109.4 \\
\hline 1999 & 112.4 & 112.8 & 108.6 & 114.6 & 111.3 \\
\hline \multicolumn{6}{|c|}{ (End of period) } \\
\hline 1994 & 107.2 & 111.7 & 108.9 & 104.2 & 104.5 \\
\hline 1995 & 108.1 & 111.7 & 109.4 & 106.5 & 105.6 \\
\hline 1996 & 110.6 & 113.6 & 108.8 & 110.2 & 108.4 \\
\hline 1997 & 110.1 & 112.6 & 1064 & 110.6 & 108.1 \\
\hline 1998 & 111.6 & 112.3 & 108.4 & 114.7 & 109.9 \\
\hline 1999 & 113.3 & 114.1 & 107.8 & 114.5 & 112.7 \\
\hline \multicolumn{6}{|l|}{1994} \\
\hline March & 105.8 & 108.3 & 112.8 & 103.5 & 103.9 \\
\hline June & 106.4 & 110.1 & 112.5 & 103.3 & 104.0 \\
\hline September & 106.7 & 110.7 & 110.4 & 103.7 & 104.3 \\
\hline December & 107.2 & 111.7 & 108.9 & 104.2 & 104.5 \\
\hline \multicolumn{6}{|l|}{1995} \\
\hline March & 107.0 & 109.5 & 110.5 & 104.9 & 105.4 \\
\hline June & 107.7 & 110.3 & 110.6 & 105.5 & 106.2 \\
\hline September & 108.0 & 111.3 & 109.1 & 105.4 & 106.2 \\
\hline December & 108.1 & 111.7 & 109.4 & 106.5 & 105.6 \\
\hline \multicolumn{6}{|l|}{1996} \\
\hline March & 108.4 & 111.3 & 110.2 & 107.0 & 106.4 \\
\hline June & 108.3 & 110.2 & 107.6 & 107.9 & 106.9 \\
\hline September & 109.1 & 111.9 & 109.3 & 108.7 & 106.9 \\
\hline December & 110.6 & 113.6 & 108.8 & 110.2 & 108.4 \\
\hline \multicolumn{6}{|l|}{1997} \\
\hline March & 109.9 & 111,9 & 108.2 & 109.7 & 108.7 \\
\hline June & 110.3 & 112.0 & 106.5 & 110.5 & 109.1 \\
\hline September & 110.6 & 112.7 & 104.4 & 110.8 & 109.4 \\
\hline December & 110.1 & 112.6 & 106.4 & 110.6 & 108.1 \\
\hline \multicolumn{6}{|l|}{1998} \\
\hline March & 110.4 & 112,2 & 108.3 & 110.5 & 108.9 \\
\hline June & 110.8 & 112.7 & 108.2 & 110.5 & 109.7 \\
\hline September & 111.5 & 112.7 & 108.1 & 113.2 & 110.0 \\
\hline December & 111.6 & 112.3 & 108.4 & 114.7 & 109.9 \\
\hline \multicolumn{6}{|l|}{1999} \\
\hline March & 112.0 & 112.6 & 108.9 & 114.5 & 110.4 \\
\hline June & 112.3 & 112.4 & 109.2 & 114.8 & 111.3 \\
\hline September & 112.6 & 112.4 & 108.9 & 114.8 & 112.2 \\
\hline December & 113.3 & 114.1 & 107.8 & 114.5 & 112.7 \\
\hline
\end{tabular}

Sources: Office of the Comptroller General; and Fund staff estimates. 
Table 15. Panama: Wholesale Price Index

\begin{tabular}{|c|c|c|c|c|}
\hline \multicolumn{5}{|c|}{$(1987=100)$} \\
\hline & Total & Imports & Industrial & Agricultural \\
\hline \multicolumn{5}{|c|}{ (In percent) } \\
\hline Weights & 100.0 & 43.7 & 45.1 & 11.2 \\
\hline \multicolumn{5}{|c|}{ (Period average) } \\
\hline 1994 & 112.4 & 116.9 & 107.9 & 113.5 \\
\hline 1995 & 115.7 & 121.5 & 110.8 & 113.2 \\
\hline 1996 & 118.2 & 126.2 & 112.3 & 111.0 \\
\hline 1997 & 115.5 & 121.0 & 111.2 & 111.7 \\
\hline 1998 & 111.0 & 114.1 & 107.6 & 113.4 \\
\hline \multicolumn{5}{|c|}{ (End of period) } \\
\hline 1994 & 114.1 & 118.6 & 109.8 & 114.4 \\
\hline 1995 & 116.2 & 121.9 & 111.4 & 113.2 \\
\hline 1996 & 120.3 & 129.2 & 113.8 & 112.2 \\
\hline 1997 & 115.6 & 121.1 & 111.0 & 113.1 \\
\hline 1998 & 111.1 & 114.5 & 107.2 & 114.8 \\
\hline \multicolumn{5}{|l|}{1995} \\
\hline March & 114.7 & 120.0 & 110.2 & 112.2 \\
\hline June & 116.0 & 121.8 & 111.1 & 113.7 \\
\hline September & 116.0 & 122.4 & 110.6 & 113.6 \\
\hline December & 116.2 & 121.9 & 111.4 & 113.2 \\
\hline \multicolumn{5}{|l|}{1996} \\
\hline March & 117.8 & 126.3 & 111.6 & 109.9 \\
\hline June & 117.0 & 124.3 & 111.4 & 111.0 \\
\hline September & 117.6 & 125.0 & 112.2 & 1110 \\
\hline December & 120.3 & 129.2 & 113.8 & 112.2 \\
\hline \multicolumn{5}{|l|}{1997} \\
\hline March & $\$ 15.8$ & 121.3 & 111.7 & 111.0 \\
\hline June & 115.4 & 120.8 & 111.2 & 111.1 \\
\hline September & 115.3 & 120.7 & 111.0 & 111.7 \\
\hline December & 115.6 & 121.1 & 111.0 & 113.1 \\
\hline \multicolumn{5}{|l|}{1998} \\
\hline March & 111.6 & 115.1 & 108.1 & 112.8 \\
\hline June & 111.1 & 113.9 & 108.0 & 113.2 \\
\hline September & 110.2 & 112.9 & 107.1 & 112.8 \\
\hline December & 111.1 & 114.5 & 107.2 & 114.8 \\
\hline \multicolumn{5}{|l|}{1999} \\
\hline March & 109.3 & 112.1 & 105.6 & 113.6 \\
\hline June & 111.5 & 112.1 & 105.6 & 113.6 \\
\hline September & 116.4 & 118.6 & 104.3 & 114.1 \\
\hline
\end{tabular}

Sources: Office of the Comptroller General; and Fund staff estimates. 
Table 16. Panama: Selected Energy Prices

\begin{tabular}{|c|c|c|c|c|c|}
\hline & 1994 & 1995 & 1996 & 1997 & 1998 \\
\hline \multicolumn{6}{|c|}{ (Balboas per megawatt hour) } \\
\hline \multicolumn{6}{|l|}{ Electricity rates $1 /$} \\
\hline Weighted average & 115.5 & 115.5 & 112.8 & 111,6 & $\cdots$ \\
\hline Residential & 120.5 & 120.5 & 120.5 & 118.0 & $\cdots$ \\
\hline Commercial & 119.2 & 119.2 & 119.5 & 118.1 & $\ldots$ \\
\hline Industrial & 100.7 & 99.4 & 99.4 & 99.4 & ... \\
\hline Govemment & 103.4 & 112.7 & 111.6 & 111.0 & .. \\
\hline \multicolumn{6}{|c|}{ (Balboas per gallon) } \\
\hline \multicolumn{6}{|l|}{ Petroleum prices } \\
\hline Gasoline premium & 1.6 & 1.7 & 1.8 & 1.8 & 1.6 \\
\hline Gasoline regular & 1.5 & 1.6 & 1.7 & 1.7 & 1.5 \\
\hline Diesel & 1.1 & 1.2 & 1.3 & 1.3 & 1.1 \\
\hline Kerosene & 1.1 & 1,2 & 1.2 & 1.1 & 1.1 \\
\hline
\end{tabular}

Sources: Hydraulic Resources and Electricity Institute (IRHE); Ministry of Commerce and Industry; and Fund staff estimales.

1/ Panama's electricity system has recently been privatized. The price survey in operation under the old regime no longer applies, and a new one has not yet been instituted. 
Table 17. Panama: Operations of the Nonfinancial Public Sector

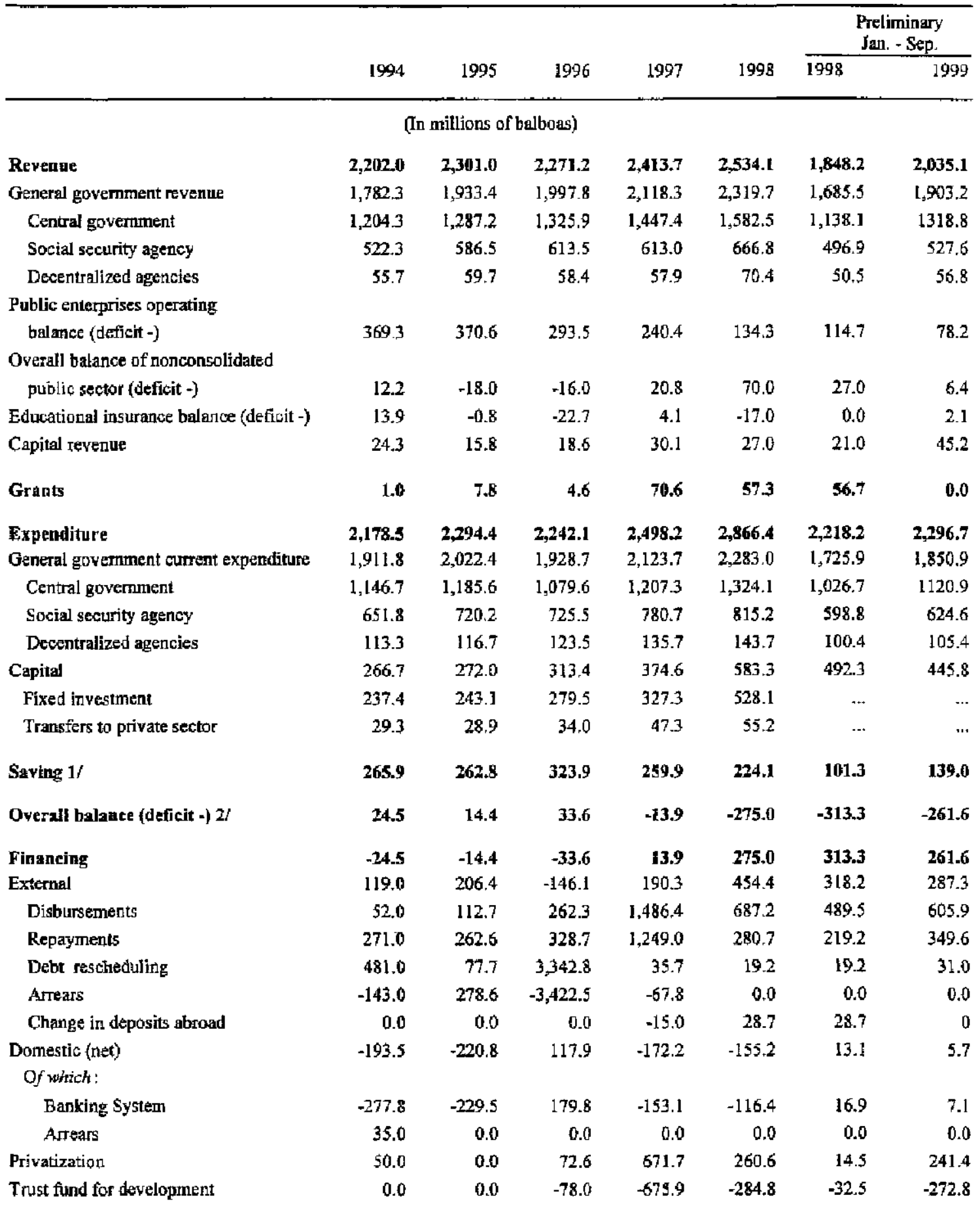


Table 17. Panama: Operations of the Nonfinancial Public Sector (Concluded)

\begin{tabular}{|c|c|c|c|c|c|c|c|}
\hline & \multirow[b]{2}{*}{1994} & \multirow[b]{2}{*}{1995} & \multirow[b]{2}{*}{1996} & \multirow[b]{2}{*}{1997} & \multirow[b]{2}{*}{1998} & \multicolumn{2}{|c|}{$\begin{array}{c}\text { Preliminary } \\
\text { Jan. - Sep. }\end{array}$} \\
\hline & & & & & & 1998 & 1999 \\
\hline \multicolumn{8}{|c|}{ (Annual percent change) } \\
\hline Revorue & 7.1 & 4,5 & -1.3 & 6.3 & 5.0 & 5.5 & 11.7 \\
\hline Expenditare & 1.0 & 5.3 & -2.3 & 11.4 & 14.7 & 18,3 & 4.9 \\
\hline Current & 2.3 & 5.8 & -4.6 & 10.1 & 7.5 & 22.1 & 7.3 \\
\hline Capital & -7.7 & 2.0 & 15.2 & I9.5 & 55.7 & ] 2.2 & -3.6 \\
\hline \multicolumn{8}{|c|}{ (In percent of GDP $3 /$ ) } \\
\hline Revenue & 28.5 & 29.1 & 27.9 & 27.9 & 27.7 & 26.9 & 28.7 \\
\hline Grants & 0.0 & 0.1 & B.1 & 0.8 & 0.6 & 0.8 & 0.0 \\
\hline Expenditure & 28.2 & 29.0 & 27.5 & 28.9 & 31.3 & 32.3 & 32.3 \\
\hline Cument & 24.7 & 25.6 & 23.7 & 24.5 & 25.0 & 25.2 & 25.7 \\
\hline Capital & 3.4 & 3.4 & 3.8 & 4.3 & 6.4 & 7.2 & 6.6 \\
\hline Saring $1 /$ & 3.4 & 3.3 & 4.0 & 3.6 & 2.4 & 1.5 & 1.9 \\
\hline Overall balance (deficit-) $2 /$ & 0.3 & 0.2 & 0.4 & $-0,2$ & -3.0 & -4.6 & +3.6 \\
\hline Financing (net) & -0.3 & -0.2 & -0.4 & 0.2 & 3.0 & 4.6 & 3.6 \\
\hline External & 1.5 & 2.6 & -1.8 & 22 & 5.0 & 4.5 & 4.0 \\
\hline Domestic (net) & -2.5 & -2.8 & 1.4 & -2.0 & -1.7 & 0.2 & 0.1 \\
\hline Privatization & 0.6 & 0.0 & 0.9 & 7.8 & 2.9 & 0.2 & 3.4 \\
\hline Trust fund for development & 0.0 & 0.0 & -1.0 & -7.8 & -3.1 & -0.5 & -7.8 \\
\hline External interest obligations & 4.6 & 5.0 & 2.9 & 3.2 & 3.2 & 1.9 & 2.0 \\
\hline \multicolumn{8}{|c|}{ (In milltons of balboas) } \\
\hline \multicolumn{8}{|l|}{ Memorandnm items: } \\
\hline External interest obligations & 402.3 & 393.0 & 241.6 & 281.6 & 288.4 & 132.0 & 146.5 \\
\hline GDP (market prices) & 7.733 .9 & $7,906.1$ & $8,151.1$ & $8,657.5$ & $9,143.8$ & $6,858.0$ & $7,203.0$ \\
\hline Balance in Trust Fund for Development & 0.0 & 0.0 & 78.0 & 754.1 & $1,038.9$ & 769.6 & $1,303.0$ \\
\hline
\end{tabular}

Sources: Office of the Comptroller General; Ministry of Economy and Finance; and Fund staff estimates

1/ Current revenue less current expenditure.

2/ Revenue and grants, less expenditure.

3/ Part-year percentages are annualized.

4/ The Trust Fund for Development invests divestment proceeds abroad and transfers its investment income to the central government to finance development projects, including social projects. 
Table 18. Panama: Central Government Operations

\begin{tabular}{|c|c|c|c|c|c|c|c|}
\hline & \multirow[b]{2}{*}{1994} & \multirow[b]{2}{*}{1995} & \multirow[b]{2}{*}{1996} & \multirow[b]{2}{*}{1997} & \multirow[b]{2}{*}{1998} & \multicolumn{2}{|c|}{$\begin{array}{c}\text { Preliminary } \\
\text { Jan,-Sept. }\end{array}$} \\
\hline & & & & & & 1998 & 1959 \\
\hline \multicolumn{8}{|c|}{ (In millions of balbogs) } \\
\hline Revenue & $1,361.9$ & $1,473 . T$ & $1,544,6$ & $1,600.4$ & 1.709 .8 & $1,208.5$ & $1,371.5$ \\
\hline Tax revenue & 916.3 & 990.2 & 984.9 & $1,043.5$ & $1,103.9$ & 816.9 & 873.5 \\
\hline Income tax & 352.4 & 405.4 & 392.4 & 416.9 & 399.5 & 291.4 & 354.8 \\
\hline Wealth taxes & 41.4 & 43.6 & 45.4 & 47.1 & 51.7 & 41.3 & 41.8 \\
\hline Taxes on foreign trode & 177.7 & 188.3 & 193.6 & 219.0 & 251.4 & 178.1 & 167.9 \\
\hline Taxes on domestic transactions & 344.8 & 352.9 & 353.5 & 360.5 & 401.3 & 306.1 & 309.0 \\
\hline Nontax revenue & 442.7 & 481.6 & 558.2 & 554.3 & 603.9 & 390.6 & 494,9 \\
\hline Panama Canal & 81.2 & 92.0 & 94.1 & 95.4 & 96.3 & 72.0 & 86.3 \\
\hline Oil pipejine royalties & 3.0 & 3.1 & 0.0 & 0.0 & 0.0 & 0.0 & 0.0 \\
\hline Transfers from the rest of public sector & 220.9 & 264.4 & 345.9 & 257.6 & $2,23.1$ & 134.3 & 113.2 \\
\hline Other & 140.6 & 122.1 & 158.2 & 201.3 & 284.5 & 184.3 & 295.4 \\
\hline Capitai revenue & 2.9 & 1.9 & 1.5 & 2.5 & 2.0 & 1.0 & 3.1 \\
\hline Grants & 1.0 & 7.8 & 4.6 & 70.6 & 57.3 & 56.7 & 0.0 \\
\hline Expenditure & $1,599.3$ & $1,627.4$ & $1,487.2$ & $1,667.5$ & $2,228.5$ & $1,787,9$ & $1,744,3$ \\
\hline Current & $1,427.7$ & $1,474.0$ & $1,327.1$ & $1,480.5$ & $1,797.9$ & $1,394.6$ & $1,353.9$ \\
\hline Wages and salaries & 533.0 & 542.9 & 568.5 & 5924 & 636.8 & 476.4 & 465.1 \\
\hline Goods and Services. & 134.1 & 111.8 & 112.5 & 147.5 & 144.3 & 114.4 & 128.5 \\
\hline Pensions and transfers & 405.6 & 417.2 & 385.7 & 394.1 & 648.1 & 505.8 & 372.9 \\
\hline \multicolumn{8}{|l|}{ Of which: } \\
\hline Social security agency & 212.2 & 216.7 & 170.7 & 194.0 & 400.0 & 335.5 & 308.7 \\
\hline Decentralized agencies & 68.8 & 68.9 & 74.5 & 76.6 & 78.4 & 59.0 & 63.2 \\
\hline Public enterprises & 0.0 & 2.8 & 2.2 & 2.6 & 20 & 0.9 & 1.0 \\
\hline Interest & 350.3 & 394.4 & 251.4 & 299.7 & 321.9 & 2612 & 351.0 \\
\hline Internal & 24.2 & 25.3 & 28.6 & 32.9 & 42.4 & 29.3 & 57.3 \\
\hline External & 326.1 & 369.1 & 222.8 & 266.8 & 279.5 & 231.9 & 293.7 \\
\hline Other curent expenditure & 4.8 & 7.7 & 8.9 & 46.9 & 46.8 & 36.8 & 36.4 \\
\hline Capital & 171.6 & 153.4 & 160.1 & I 87.0 & 430.6 & 393.3 & 390.4 \\
\hline Fixed capital formation & 115.6 & 113.6 & 117,8 & 131.9 & 381.7 & 334.0 & 358.3 \\
\hline Transfers of capital & 56.0 & 39.8 & 42.3 & 55.1 & 29.2 & 40.1 & 32.1 \\
\hline To decentralized agencies & 7.9 & 10.8 & 6.4 & 2.4 & 3.5 & 0.0 & 0.1 \\
\hline To public enterprises & 18.8 & 0.1 & 1.9 & 5.5 & 0.2 & 0.0 & 0.0 \\
\hline Other & 29.3 & 28.9 & 34.0 & 47.2 & 25.5 & 40.1 & 320 \\
\hline On feuding & 0.0 & 0.0 & 48.0 & -30.0 & 0.0 & n.t) & 0.0 \\
\hline Saving $I /$ & -68.7 & -2.2 & 216.0 & 117.4 & -90.1 & -187.1 & 14.5 \\
\hline Overall balance (deficit-) $2 /$ & -236.4 & -145.9 & 14.0 & 3.5 & -461.4 & .522 .7 & -3728 \\
\hline
\end{tabular}


Table 18. Panama: Central Government Operations (Concluded)

\begin{tabular}{|c|c|c|c|c|c|c|c|}
\hline & \multirow[b]{2}{*}{1994} & \multirow[b]{2}{*}{1995} & \multirow[b]{2}{*}{1996} & \multirow[b]{2}{*}{1997} & \multirow[b]{2}{*}{1998} & \multicolumn{2}{|c|}{$\begin{array}{l}\text { Preliminary } \\
\text { Jan.-Sept. }\end{array}$} \\
\hline & & & & & & 1998 & 1999 \\
\hline \multicolumn{8}{|c|}{ (In millions of balboas) } \\
\hline Finarcing & 236.4 & 145.9 & -14.0 & -3.5 & 461.4 & 522.7 & 372.8 \\
\hline External & 147.0 & 269.8 & -96.9 & 240.1 & 471.8 & $3 \div 8.4$ & 295.7 \\
\hline Disbursements & 58.0 & 99.5 & 250.6 & $1,483,1$ & 655.0 & 457.3 & 603.2 \\
\hline Repapments & 198.0 & 1960 & 274.6 & $1,195.9$ & 231.1 & 186.8 & 336.6 \\
\hline Debt rescheduling & 481.0 & 29.9 & $3,342.8$ & 35.7 & 192 & 19.2 & 29.1 \\
\hline Anears & -144.0 & 336.4 & $-3,415.7$ & -67.8 & 0.0 & 0.0 & 0.0 \\
\hline Change in deposits abroad & & 0.0 & 0.0 & -15.0 & 28.7 & 28.7 & 0.0 \\
\hline Domestic (net) & 39.4 & -123.9 & 88.3 & -243.6 & -10.5 & 189.8 & .164 .3 \\
\hline Of which: & & & & & & & \\
\hline Banking system & -12.6 & -55.9 & 222.0 & -132.3 & -159.2 & 118.5 & 23.6 \\
\hline Arrears & 35.0 & 0.0 & 0.0 & 0.0 & 0.0 & 0.0 & 0.0 \\
\hline Privatization & 50.0 & 0.0 & 726 & 671.7 & 260.5 & 14.5 & 241.4 \\
\hline Trust Fund for Development & 0.0 & 0.0 & -780 & -671.7 & -260.5 & -14.5 & .241 .4 \\
\hline \multicolumn{8}{|c|}{ (Annual percent change) } \\
\hline Current revenue & 0.4 & 8.2 & 4.8 & 3.6 & 6.8 & 4.2 & 18.9 \\
\hline Tax revenue & 62 & 8.1 & -0.5 & 5.9 & 5.8 & 1.7 & 6.9 \\
\hline Nontax revenuse & -9.9 & 8.8 & 15.9 & -0.7 & 8.9 & 6.8 & 26.7 \\
\hline Total expenditure & 0.5 & 1.8 & -8.6 & 12.1 & 33.6 & 28.3 & -2.4 \\
\hline Current expenditure & 1.9 & 3.2 & -10.0 & 11.6 & 21.4 & 18.9 & -2.9 \\
\hline Capital expenditure & -16.5 & -10.6 & 4.4 & 16.8 & 130,3 & 74.2 & -0.7 \\
\hline \multicolumn{8}{|c|}{ (In percent of GDP)3/ } \\
\hline Curtent revenute & 17.6 & 18.6 & 18.9 & 18.5 & 18.7 & 17.6 & 19.8 \\
\hline Tax sevenus & 11.8 & 12.5 & 12.1 & 12.1 & 12.1 & 11.9 & 12.1 \\
\hline Nontmx revesue & 5.7 & 6.1 & 6.8 & 6.4 & 6.6 & 5.7 & 6.9 \\
\hline Totsl expenditure & 20.7 & 20.6 & 18.2 & 19.3 & 24.4 & 26.1 & 24.2 \\
\hline Current expenditure & 18.5 & 18.6 & 16.3 & 17.1 & 19.7 & 20.3 & 18.8 \\
\hline Capita] expenditure & 2.2 & 1.9 & 2.0 & 2.2 & 4.7 & 5.7 & 5.4 \\
\hline Saving $1 /$ & -0.9 & 0.0 & 2.6 & 1.4 & -1.0 & -2.7 & 02 \\
\hline Overall balance (deficit -) $2 /$ & -3.1 & -1.8 & 0.2 & 0.6 & -5.0 & -7.6 & -52 \\
\hline Financing (net) & 3.1 & 1.8 & -0.2 & 0.0 & 5.0 & 7.6 & 5.2 \\
\hline External & 1.9 & 3.4 & -1.2 & 2.8 & 5.2 & 4.6 & 4.1 \\
\hline Domestic & 0.5 & -1.6 & 1.1 & -2.8 & -0.1 & 2.8 & -2.3 \\
\hline Privatizetion & 0.6 & 0.0 & 0.9 & 7.8 & 2.8 & 0.2 & 3.4 \\
\hline Trust Fund for Development & 0.0 & 0.0 & .1 .0 & +7.8 & -2.8 & -0.2 & -3.4 \\
\hline \multicolumn{8}{|c|}{ (In miltions of balboas) } \\
\hline \multicolumn{8}{|l|}{ Memorandem items: } \\
\hline GDP (market prices) & $7,733.9$ & $7,906.1$ & $8,151.1$ & $8,657.5$ & $9,143.8$ & $6,857.9$ & $7,203.0$ \\
\hline Balance in Trust Fund for Development 4 & 0.0 & 0.0 & 78.0 & 754.1 & $1,038.9$ & 769.6 & $1,303,0$ \\
\hline
\end{tabular}

Sources: Office of the Comptroller General; Ministry of Economy and Finance; and Fund staff estimates

1/ Current revenue less current expenditure.

2/ Revenue and grants, less expenditure.

3/ Part-year percentages are annualized.

4/ The Trust Fund for Development invests divestrnent proceeds abroad and transfers its investment income to the ceatral government to finance development projects, including social projects. 
Table 19. Panama: Central Government Revenue

\begin{tabular}{|c|c|c|c|c|c|c|c|}
\hline & \multirow[b]{2}{*}{1994} & \multirow[b]{2}{*}{1995} & \multirow[b]{2}{*}{1996} & \multirow[b]{2}{*}{1997} & \multirow[b]{2}{*}{1998} & \multicolumn{2}{|c|}{$\begin{array}{l}\text { Preliminary } \\
\text { Jan.-Sept. }\end{array}$} \\
\hline & & & & & & 1998 & 1999 \\
\hline \multicolumn{8}{|c|}{ (In millions of balboes) } \\
\hline Total revenue (including grants) & $1,362.9$ & $1,481.5$ & $1,549.2$ & $1,670.9$ & $1,766.4$ & $1,265.2$ & $1,371.5$ \\
\hline $\begin{array}{l}\text { Tax revenue } \\
\text { Direct taxes }\end{array}$ & $\begin{array}{l}916.3 \\
393.8\end{array}$ & $\begin{array}{l}990.2 \\
449.0\end{array}$ & $\begin{array}{l}984.9 \\
437.8\end{array}$ & $\begin{array}{r}1,043.5 \\
464.0\end{array}$ & $\begin{array}{r}1,103.9 \\
451.2\end{array}$ & $\begin{array}{l}816.9 \\
332.7\end{array}$ & $\begin{array}{l}\mathbf{8 7 3 . 5} \\
396.6\end{array}$ \\
\hline Incone tax & 352.4 & 405.4 & 392.4 & 416.9 & 399.5 & 291.4 & 354.8 \\
\hline Wealth tax & 41.4 & 43.6 & 45.4 & 47.1 & 51.7 & 41.3 & 41.8 \\
\hline Taxes on fareign trade & $\mathrm{t} 77.7$ & 188.3 & 193.6 & 219.0 & 251.4 & 178.1 & 167.6 \\
\hline Export taxes & t3.6 & 10.7 & 9.4 & 6.2 & 29 & 2,3 & 0.5 \\
\hline Emport taxes. & 164.1 & 177.6 & 184.2 & 212.8 & 248.5 & 175.8 & 167.1 \\
\hline Taxes on domestic transactions & 344.8 & 352.9 & 353.5 & 360.5 & 401.3 & 306.1 & 309.0 \\
\hline Tobacco and beverages & 40.8 & 41.4 & 37.1 & 40.2 & 38.0 & 29.6 & 25.0 \\
\hline Value added tax & 143.2 & 147.3 & 154.6 & 163.5 & 182.5 & 136.9 & 140.7 \\
\hline Petrcleum products & 93.9 & 95.3 & 97.6 & 93.9 & 115.2 & 86.3 & 93.4 \\
\hline Stamp texes & 36.3 & 37.6 & 33,6 & 37.3 & 38,4 & 27.3 & 28.1 \\
\hline Other & 30.6 & 31.2 & 30.7 & 25.6 & 27.2 & 26.0 & 21.8 \\
\hline $\begin{array}{l}\text { Nontax revenue } \\
\text { Panama Canal }\end{array}$ & $\begin{array}{r}442.7 \\
81.2\end{array}$ & $\begin{array}{r}481.6 \\
92.0\end{array}$ & $\begin{array}{r}558.2 \\
94.1\end{array}$ & $\begin{array}{r}594.3 \\
95.4\end{array}$ & $\begin{array}{r}603.8 \\
96.3\end{array}$ & $\begin{array}{r}390.6 \\
72.0\end{array}$ & $\begin{array}{r}494.9 \\
86.3\end{array}$ \\
\hline Oll pipeline royalties & 3.0 & 3.1 & 0.0 & 0.0 & 0.0 & 0.0 & 0.0 \\
\hline Other Services & 137.6 & 122.1 & 118.2 & 181.0 & 212.3 & 132.3 & 154.5 \\
\hline $\begin{array}{l}\text { Transfers form rest of public sector } \\
\text { Of which: }\end{array}$ & 220.9 & 264.4 & 345.9 & 257.6 & $223 . !$ & 134.3 & 113.2 \\
\hline Consolidated public sector & 154.7 & 184.7 & 217.2 & 150.4 & 125.9 & 69.4 & 50.3 \\
\hline Nanconsolidated public sector & 11.6 & 13.6 & 64.3 & 107.2 & 97.2 & 64.9 & 62.9 \\
\hline Interest earnings and dividends & 0.0 & 0.0 & 0.0 & 20.3 & 72.1 & 51.9 & 141.0 \\
\hline Capital revenne & 2.9 & 1.9 & 1.5 & 2.5 & 1.4 & 1.0 & 3.1 \\
\hline Grants & 1,0 & 7.8 & 4.6 & 70.6 & 57.3 & 56.7 & 0.0 \\
\hline \multicolumn{8}{|c|}{ (In percent of GDP 1/) } \\
\hline Total revenue & 17.6 & 18.7 & 19.0 & 19.3 & 19.3 & 18.4 & 19.0 \\
\hline Tax revenue & 11.8 & 12.5 & 12.1 & 12.1 & 12.1 & 11.9 & 12.1 \\
\hline Direct & 5.1 & 5.7 & 5.4 & 5.4 & 4.9 & 4.9 & 5.5 \\
\hline Income tax & 4.6 & 5.1 & 4.8 & 4.8 & 4.4 & 4.2 & 4.9 \\
\hline Wealth tax & 0.5 & 0.6 & 0.6 & 0.5 & 0.6 & 0.6 & 0.6 \\
\hline Taxes on foreign trade & 2.3 & 2.4 & 2.4 & 2.5 & 2.7 & 2.6 & 2.3 \\
\hline Export taxes & 0.2 & 0.1 & 0.1 & 0.1 & 0.0 & 0.0 & 0.0 \\
\hline Import taxes & 2.1 & 2.2 & 2.3 & 2.5 & 2.7 & 2.6 & 2.3 \\
\hline Taxes on domestic transactions & 4.5 & 4.5 & 4.3 & 4.2 & 4.4 & 4.5 & 4.3 \\
\hline Tabacco and beverages & 0.5 & 0.5 & 0.5 & 0.5 & 0.4 & 0.4 & 0.3 \\
\hline Value added tax & 1.9 & 1.9 & 1.9 & 1.9 & 2.0 & 2.0 & 2.0 \\
\hline Petroleum products & 1.2 & 1.2 & 1.2 & 1.1 & 1.3 & 1.3 & 1.3 \\
\hline Stamp taxes & 0.5 & 0.5 & 04 & 0.4 & 0.4 & 0.4 & 0.4 \\
\hline Other & 0.4 & 0.4 & 0.4 & 0.3 & 0.3 & 0.4 & 0.3 \\
\hline Nonfax revende & 5.7 & 6.1 & 6.8 & 6.4 & 6.6 & 5.7 & 6.9 \\
\hline Panama Canal & 1.0 & 1.2 & 1.2 & 1.1 & 1.1 & 1.0 & 1.2 \\
\hline Oil pipeline royalties & 0.0 & 0.0 & 0.0 & 0.0 & 0.0 & 0.0 & 0.0 \\
\hline Serviceses & 1.8 & 1.5 & 1.5 & 2.1 & 2.3 & 1.9 & 2.1 \\
\hline $\begin{array}{l}\text { Transfers from rest of public sector } \\
\text { of which: }\end{array}$ & 2.9 & 3.3 & 4.2 & 3.0 & 2.4 & 2.0 & 1.6 \\
\hline Consolidated public sector & 2.0 & 2.3 & 2.7 & 1.7 & 1.4 & 1.0 & 0.7 \\
\hline Noncansolidated public sector & 0.1 & 0.2 & 0.8 & 1.2 & 1.1 & 0.9 & 0.9 \\
\hline Interest earnings and dividernds & 0.0 & 00 & 0.0 & 0.2 & 0.8 & 0.8 & 2.0 \\
\hline Capital tevenue & 0.0 & 0.0 & 0.0 & 0.0 & 0.0 & 0.8 & 0.0 \\
\hline Grants & 0.0 & 0.1 & B.1 & 0.8 & 0.6 & 0.8 & 0.0 \\
\hline
\end{tabular}

Sources: Office of the Comptroller General; Ministry of Economy and Finance; and Fund staff estimates.

1/ Part-year percentages are annualized. 
Table 20. Panama: Central Government Expenditure

\begin{tabular}{|c|c|c|c|c|c|c|c|}
\hline & \multirow[b]{2}{*}{1994} & \multirow[b]{2}{*}{1995} & \multirow[b]{2}{*}{1996} & \multirow[b]{2}{*}{1997} & \multirow[b]{2}{*}{1998} & \multicolumn{2}{|c|}{$\begin{array}{c}\text { Preliminary } \\
\text { Jan.-Sep. }\end{array}$} \\
\hline & & & & & & 1998 & 1999 \\
\hline \multicolumn{8}{|c|}{ (In percent of GDP $1 /$ ) } \\
\hline Total expenditure $2 /$ & 20.7 & 20.2 & 19.6 & 18.5 & 17.5 & 26.1 & 24.2 \\
\hline Current expenditure & 18.5 & 18.6 & 16.3 & $17+1$ & 19.7 & 20.3 & 18.8 \\
\hline Wages and solaries & 6.9 & 6.9 & 7.0 & 6.8 & 7.0 & 6.9 & 6.5 \\
\hline Goods and services & 1.7 & 1.4 & 1.4 & 1.7 & 1.6 & 1.7 & 1.8 \\
\hline Interest & 4.5 & 5.0 & 3.1 & 3.5 & 3.5 & 3.8 & 4.9 \\
\hline Pensions and transfers & 5.2 & 5.3 & 4.7 & 4.6 & 7.1 & 7.4 & 5.2 \\
\hline Other & 0.1 & 0.1 & 0.1 & 0.5 & 0.5 & 0.5 & 0.5 \\
\hline Capital expenditure & 2.2 & 1.9 & 2.0 & 2.2 & 4,5 & 5.7 & 5.4 \\
\hline Investment & 1.5 & 1.4 & 1.4 & 1.5 & 4.2 & 4.9 & 5.0 \\
\hline Transfers & 0.7 & 0.5 & 0.5 & 0.6 & 0.3 & 0.9 & 0.4 \\
\hline \multicolumn{8}{|l|}{ Memorandum item: } \\
\hline Noninterest expenditure & 16.1 & 15.6 & 15.2 & 15.8 & 20.6 & 22.3 & 19.3 \\
\hline \multicolumn{8}{|c|}{ (Annual percent change) } \\
\hline Total expenditure & -0.5 & I.8 & -8.6 & 12.1 & 33.6 & 22.0 & 29.4 \\
\hline Currest expenditure & 1.9 & 3.2 & -10.0 & 11.6 & 21.4 & 14.5 & 27.8 \\
\hline Wages and salaries & -6.1 & 1.9 & 4.7 & 4.2 & 7.5 & 5.5 & 9.5 \\
\hline Goods and services & -10.2 & -16.6 & 0.6 & 31.1 & -2.2 & 27.3 & 27.8 \\
\hline Interest & 20.5 & 12.6 & -36.3 & 19.2 & 7.4 & -12.6 & 10.0 \\
\hline Pensions and transfers & 4.3 & 2.9 & -7.5 & 2.2 & 64.5 & 40.8 & 63.5 \\
\hline Other & -4.0 & 60.3 & 15.7 & 427.0 & -0.2 & 489.3 & 10.3 \\
\hline Copital expenditure & -16.5 & -10.6 & 4.4 & 16.8 & 130.3 & 103.0 & 38.4 \\
\hline Investment & -28.8 & -1.7 & 3.7 & 12.0 & 189.4 & 114.2 & 44.3 \\
\hline Transfers & 30.2 & -28.9 & 6.3 & 30.3 & -47.0 & 72.6 & 18.2 \\
\hline \multicolumn{8}{|l|}{ Memorandum item: } \\
\hline Noninterest expenditure & -5.1 & -5.1 & -5.1 & -5.1 & -5.1 & 32.5 & 33.1 \\
\hline
\end{tabular}

Sources: Office of the Comptroller Genergl; Ministry of Economy and Finance; and Fund staff estimates.

1/ Part-year percentages are annualized. 
Table 21. Panama: Operations of the Social Security Agency

\begin{tabular}{|c|c|c|c|c|c|c|c|}
\hline & \multirow[b]{2}{*}{1994} & \multirow[b]{2}{*}{1995} & \multirow[b]{2}{*}{1996} & \multirow[b]{2}{*}{1997} & \multirow[b]{2}{*}{1998} & \multicolumn{2}{|c|}{$\begin{array}{c}\text { Preliminary } \\
\text { Jan.-Sep. }\end{array}$} \\
\hline & & & & & & $\overline{1998}$ & 1999 \\
\hline \multicolumn{8}{|c|}{ (In millions of baltooas) } \\
\hline Current revenue & 522.3 & 586.5 & 613.5 & 613.0 & 666.8 & 496.9 & $\mathbf{5 2 7 . 6}$ \\
\hline Contributions & 396.3 & 452.6 & 475.0 & 488.8 & 522.9 & 392.2 & 427.9 \\
\hline Professional risk premium & 35.0 & 40.5 & 38.5 & 36.8 & 40.6 & 29.5 & 33.4 \\
\hline Administered funds & 18.0 & 29.1 & 24.3 & 11.9 & 6.9 & 4.8 & 4.8 \\
\hline Income from investment & 39.0 & 53.8 & 59.5 & 58.5 & 64.3 & 47.1 & 46.5 \\
\hline Other & 34.0 & 10.5 & 162 & 17.0 & 32.1 & 23.3 & 15.0 \\
\hline Current expenditure & 651.8 & 720.2 & $\mathbf{7 2 5 . 5}$ & 780.6 & 815.2 & 598.8 & 624.6 \\
\hline Wages & 134.3 & 143.3 & $15[.4$ & 156.2 & 166.0 & 121.3 & 127.6 \\
\hline Goods and services & 82.2 & 107.3 & 90.6 & 97.5 & 104.2 & 76.5 & 79.3 \\
\hline Transfers & 435.3 & 468.6 & 482.6 & 526.2 & 544.4 & 400.4 & 417.3 \\
\hline Domestic interest & 0.0 & 0.9 & 1.0 & 0.7 & 0.6 & 0.6 & 0.4 \\
\hline Operating balance (deficit -) & -129.5 & -133.7 & -112.0 & -167.6 & -148.4 & -101.9 & -97.0 \\
\hline Current transfers (net) & 211.7 & 216.7 & 170.7 & 193.5 & 399.5 & 335.2 & 203.3 \\
\hline Transfers from central government & 212.2 & 216.7 & 170.7 & 194.0 & 400.0 & 335.5 & 203.6 \\
\hline Transfers to central government & 0.5 & 0.0 & 0.0 & 0.5 & 0.5 & 0.3 & 0.3 \\
\hline Capital revenue & 10.8 & 11.4 & 12.2 & 9.7 & 10.5 & 6.9 & 8.8 \\
\hline Saving 1/ & 93.0 & 94.4 & 70.9 & 35.6 & 261.6 & 240.2 & 115.1 \\
\hline Capital expenditure & .7 .8 & 20.2 & 37.1 & 24.2 & 27.0 & 19.8 & 22.0 \\
\hline Fixed investment & 7.8 & 11.6 & 20.2 & 24.2 & 27.0 & 19.8 & 22.0 \\
\hline Finascial investment & 0.0 & 8.6 & 16.9 & 0.0 & 0.0 & 0.0 & 0.0 \\
\hline Overall balance (deficit -) & 85.2 & 74.2 & 33.8 & 11.4 & 234.6 & 220.4 & 93.1 \\
\hline \multicolumn{8}{|c|}{ (In percent of GDP $2 /$ ) } \\
\hline Current revenue & 6.8 & 7.4 & 7.5 & 7.1 & 3.3 & 72 & 7.3 \\
\hline Of which: & & & & & & & \\
\hline Contributions & 5.1 & 5.7 & 5.8 & 5.6 & 5.7 & 5.7 & 5.9 \\
\hline Total expenditure & 8.5 & 9.4 & 9.4 & 9.3 & 9.2 & 9.0 & 9.0 \\
\hline \multicolumn{8}{|l|}{ Of which: } \\
\hline Cutent & 8.4 & 9.1 & 8.9 & 9.0 & 8.9 & 8.7 & 8.7 \\
\hline Operating balance (deficit -) & -1.7 & -1.7 & -1.4 & -1.9 & -1.6 & -1.5 & -1.3 \\
\hline Current transfers (net) & 2.7 & 2.7 & 2.1 & 2.2 & 4.4 & 4.9 & 2.8 \\
\hline Overall balance (deficit -) & 1.1 & 0.9 & 0.4 & 0.1 & 2.6 & 3.2 & 1.3 \\
\hline
\end{tabular}

Sources: Social Security Agency; Ministry of Economy and Finance; and Fund staff estimates.

$1 /$ Including capital revenue.

2/ Part-year percentages are annualized. 
Table 22. Panama: Operations of the Decentralized Agencies 1/

\begin{tabular}{|c|c|c|c|c|c|c|c|}
\hline & \multirow[b]{2}{*}{1994} & \multirow[b]{2}{*}{1995} & \multirow[b]{2}{*}{1996} & \multirow[b]{2}{*}{1997} & \multirow[b]{2}{*}{1998} & \multicolumn{2}{|c|}{$\begin{array}{c}\text { Preliminary } \\
\text { Jan-Sep. }\end{array}$} \\
\hline & & & & & & 1998 & 1999 \\
\hline \multicolumn{8}{|c|}{ (In millions of balbross) } \\
\hline Operating revenue & $\mathbf{5 5 . 7}$ & 59.7 & 58.4 & 57.9 & 70.4 & 50.5 & 56.8 \\
\hline Operating expenditure & 113.3 & 116.7 & 123.5 & 135.7 & 143.7 & 100.4 & 105.4 \\
\hline Interest & 7.3 & 6.7 & 6.2 & 6.7 & 9.0 & 3.7 & 4.6 \\
\hline Other & 106.0 & 110.0 & 117.4 & 129.0 & 134.7 & 96.7 & 100.8 \\
\hline Operating balance (deftcit -) & -57.6 & -56.9 & $-6 \mathbf{5 . 1}$ & -77.8 & -73.3 & $-49,9$ & 48.6 \\
\hline Corrent transfers (net) & 68.7 & 68.9 & 70.0 & 76.6 & 74.4 & 59.0 & 63.1 \\
\hline Transfers from central government & 68.8 & 68.9 & 74.5 & 76.6 & 78.4 & 59.0 & 63.2 \\
\hline Trarsfers to central government & 0.1 & 0.0 & 4.6 & 0.0 & 4.0 & 0.0 & 0.1 \\
\hline Transfers from public enterprises 2 & -0.1 & 0.0 & -9.1 & 0.0 & 0.0 & 0.0 & 0.0 \\
\hline Capital transfers from central government & 7.9 & 10.8 & 6.4 & 3.4 & 3.3 & 33 & 2.0 \\
\hline Capital revenue & 3.4 & 2.5 & 1.7 & 10.8 & 15.1 & 13.2 & 13.8 \\
\hline Saving $\mathbf{3}$ & 14.5 & 14.5 & 6.5 & 9.6 & 16.2 & 22.3 & 28.3 \\
\hline Copital expenditure & 9.6 & 11.5 & 20.3 & 23.0 & 24.5 & 19.8 & 13.4 \\
\hline Fixed investment & 19.6 & 13.6 & 15.6 & 23.0 & 24.5 & 19.8 & 13.4 \\
\hline Net lending & -10.0 & -2.1 & 4.7 & 0.0 & 0.0 & 0.0 & 0.0 \\
\hline Overall balance (deficit -) & 12.8 & 13,8 & -73 & -11.0 & $-\mathbf{5 . 0}$ & 5.8 & 16.9 \\
\hline \multicolumn{8}{|c|}{ (In percent of GDP) $4 /$} \\
\hline Operating revenue & 0.7 & 0.8 & 0.7 & 0.7 & 0.8 & 0.7 & 0.8 \\
\hline Operating expenditure & 1.5 & 1.5 & 1.5 & 1.6 & 1.6 & 1.5 & 1.5 \\
\hline Operating balance (deficit -) & -0.7 & -0.7 & -0.8 & -0.9 & -0.8 & -0.7 & -0.7 \\
\hline Curtent transfers (net) & 0.9 & 0.9 & 0.9 & 0.9 & 0.8 & 0.9 & 0.9 \\
\hline Overall balance (deficit -) & 0.2 & 0.2 & -0.1 & -0.1 & -0.1 & 0.1 & 0.2 \\
\hline
\end{tabular}

Souces: Office of the Comptrolles Gentral; Ministry of Economy and Finance; and Fund staff estimates.

I/ Includes the operations of the University of Panama, Human Development Institute (IFARFU), Agricultural Development Bank (BDA), Agricultural Marketing Institute (IMA), and National Mortgage Bark (BHN).

2/ Transfers received to finance vocational training.

3/ Inciuding cepital revenue.

4/ Part-year percentages are annualized. 
Table 23. Panama: Operations of the Public Enterprises 1f

\begin{tabular}{|c|c|c|c|c|c|c|c|}
\hline & \multirow[b]{2}{*}{1994} & \multirow[b]{2}{*}{1995} & \multirow[b]{2}{*}{1996} & \multirow[b]{2}{*}{1997} & \multirow[b]{2}{*}{1998} & \multicolumn{2}{|c|}{$\begin{array}{c}\text { Preliminary } \\
\text { Jan.-Sep. }\end{array}$} \\
\hline & & & & & & 1998 & 1999 \\
\hline \multicolumn{8}{|c|}{ (In millions of balboas) } \\
\hline Current revenue & 754.3 & 79.0 & 740.6 & 637.6 & 498.8 & 395.0 & 180.3 \\
\hline Operating expenditure & 385.0 & 408.4 & 447.2 & 397.2 & 364,5 & 280,3 & 102.2 \\
\hline Wages and salaries & 149.1 & 169.4 & 174,3 & 122.5 & 95.9 & 75.7 & 33.4 \\
\hline Goods and services & 158.7 & 164.2 & 152.3 & 127.7 & 174.5 & 120.0 & 41.8 \\
\hline Transfers & 335 & 34.3 & 73.1 & 60.6 & 25.4 & 18.5 & 13.2 \\
\hline Other & 14.7 & 15.6 & 27.8 & 59.1 & 50,2 & 49.2 & 6.5 \\
\hline Interest & 29.0 & 24.9 & 19.6 & 27.3 & 18.5 & 16.9 & 7.3 \\
\hline Domestic & $\mathbf{I} 1$ & 1.0 & 0.8 & 12.5 & 7.7 & 7.2 & 3.4 \\
\hline External & 27.9 & 23.9 & 18.8 & 14.8 & 10.8 & 9.7 & 3.9 \\
\hline Operating balance (deficit -) & 369.3 & 370.6 & 293.4 & 240.4 & 134.3 & 114.7 & 78.1 \\
\hline Current transfers net of taxes & -154.1 & -181.9 & -210.4 & -147.3 & $-1,9.4$ & -68.3 & -48.9 \\
\hline Transfers from central govemment & 0.0 & 2.8 & 2.2 & 2.6 & 2.0 & 0.9 & 1.0 \\
\hline Transfers to central government & -154.1 & -184.7 & -212.6 & -149.9 & $-121,4$ & -69.2 & -49.9 \\
\hline Transfers to rest of public sector & 0.0 & 0.0 & 0.0 & 0.0 & 0.0 & 0.0 & 0.0 \\
\hline Capital revenue & 7.2 & 0.0 & 3.3 & 7.0 & 0.0 & 0,0 & 0,0 \\
\hline Capital transfers from central government & 18.8 & 0.1 & 1.9 & 5.5 & 0.2 & 0.0 & 0,0 \\
\hline Saving $2 i$ & 222.4 & 188.7 & 86.3 & 100.1 & 14.9 & 46.4 & 29.2 \\
\hline Capital expenđiture & 104.6 & 97.8 & 104.3 & 96.2 & 104.7 & 87.6 & 12.8 \\
\hline Net borrowing & 0.0 & 0.0 & 48.0 & -30.0 & 0.0 & 0.0 & 0.0 \\
\hline Overall balance (defieit -) & 136.7 & 91.0 & 31.9 & -20.6 & -89.6 & -41.2 & 16.4 \\
\hline \multicolumn{8}{|c|}{ (In percent of GDP $3 /$ ) } \\
\hline Current revenue & 9.8 & 9.9 & 9.1 & 7.4 & 5.5 & 5.8 & 2.5 \\
\hline Operating expenditure & 5.0 & 5.2 & 5.5 & 4.6 & 4.0 & 4.1 & 1.4 \\
\hline Operating balance (deficit -) & 4.8 & 4.7 & 3.6 & 2.8 & 1.5 & 1.7 & 1.1 \\
\hline Current transfers (net) & -2.0 & -2.3 & -2.6 & -1.7 & -1.3 & -1.0 & -0.7 \\
\hline Capital experxditure & 1.4 & 1.2 & 1.3 & 1.1 & 1.1 & 1.3 & 0.2 \\
\hline Overall balance (deficit - ) & 1.8 & 1.2 & 0.4 & -0.2 & -1.0 & -0.6 & 0.2 \\
\hline
\end{tabular}

Sources: Office of the Comptroller General; Ministry of Economy and Finance; and Fund staff estimates.

1/ Includes the operations of the Hydraulic Resources and Electricity Institute (IHRE), Nationai Telecommunications Institute (NTEL) (until May 1997), Colon Free Zone (operating agency), Civil Aviation Authority, National Water and Sewerage Institute (IDAAN), La Victoria Sugar Corporation, Tourism Institute, Bayano Cement Plant (untit September 1994), and the Port Authority which on March 1, 1997 privatized about three quarters of its operations.

2/Including capital revenue.

3/ Part-year percentages are annualized. 
Table 24. Panama: Nonfinancial Public Sector Gross Domestic Debt

(In miltions of balboas; end of period)

\begin{tabular}{|c|c|c|c|c|c|c|c|}
\hline & \multirow[b]{2}{*}{1994} & \multirow[b]{2}{*}{1995} & \multirow[b]{2}{*}{1996} & \multirow[b]{2}{*}{1997} & \multirow[b]{2}{*}{1998} & \multicolumn{2}{|c|}{ September } \\
\hline & & & & & & 1998 & 1999 \\
\hline \multicolumn{8}{|c|}{ Consolidated nonfmancial } \\
\hline pablic sector & $1,003.5$ & 946.1 & $1,136.6$ & $1,168.3$ & $1,092.4$ & $1,082.0$ & $1,460.5$ \\
\hline Loang & 41.8 & 37.0 & 207.0 & 230.2 & 245.8 & 215.0 & 227,7 \\
\hline Securities & 961.7 & 909.2 & 929.5 & 938.1 & 846.7 & 867.0 & $1,232.7$ \\
\hline General government & $1,019.5$ & 964.3 & $1,159.4$ & $1,179.8$ & $1,093.7$ & $1,078.4$ & $2,456.8$ \\
\hline Loans & 6.3 & 3.6 & 177.4 & 207.2 & 230.6 & 190.0 & 203.6 \\
\hline Securities & $1,013.2$ & 960.6 & 982.0 & 972.6 & 863.1 & 888.4 & $1,253,2$ \\
\hline Central government & $1,794.7$ & $1,670.7$ & $1,792.2$ & $1,735.6$ & $1,736.0$ & $1,736.7$ & $2,149.8$ \\
\hline Loans & 31.1 & 25.8 & 196.7 & 223.2 & 243.1 & 203.5 & 213.3 \\
\hline Securities & $1,763.6$ & $1,644.9$ & $1,595.5$ & $1,512.4$ & $1,492.9$ & 1,5332 & $1,936.5$ \\
\hline \multicolumn{8}{|l|}{ Of which: } \\
\hline Short term & 15.6 & 18.5 & 82.1 & 125.0 & 38.0 & 53.1 & 90.0 \\
\hline
\end{tabular}

Sources: Office of the Comptroller General; and Fund staff estimates. 
Table 25. Panama: Accounts of the Banking System 1/

(In millions of balboas; end of period)

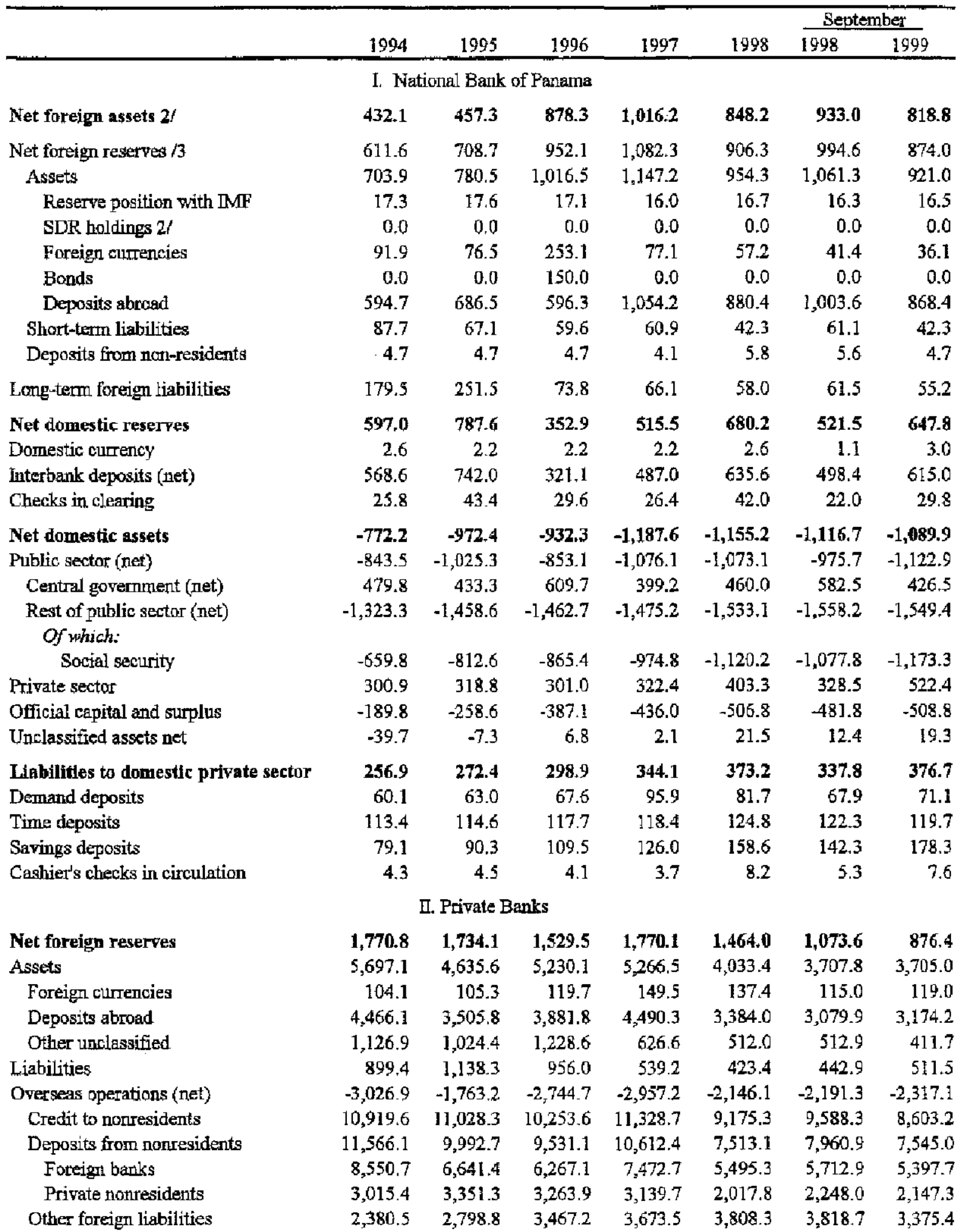


Table 25. Panama: Accounts of the Banking System 1/ (Continued)

(In millions of balboas; end of period)

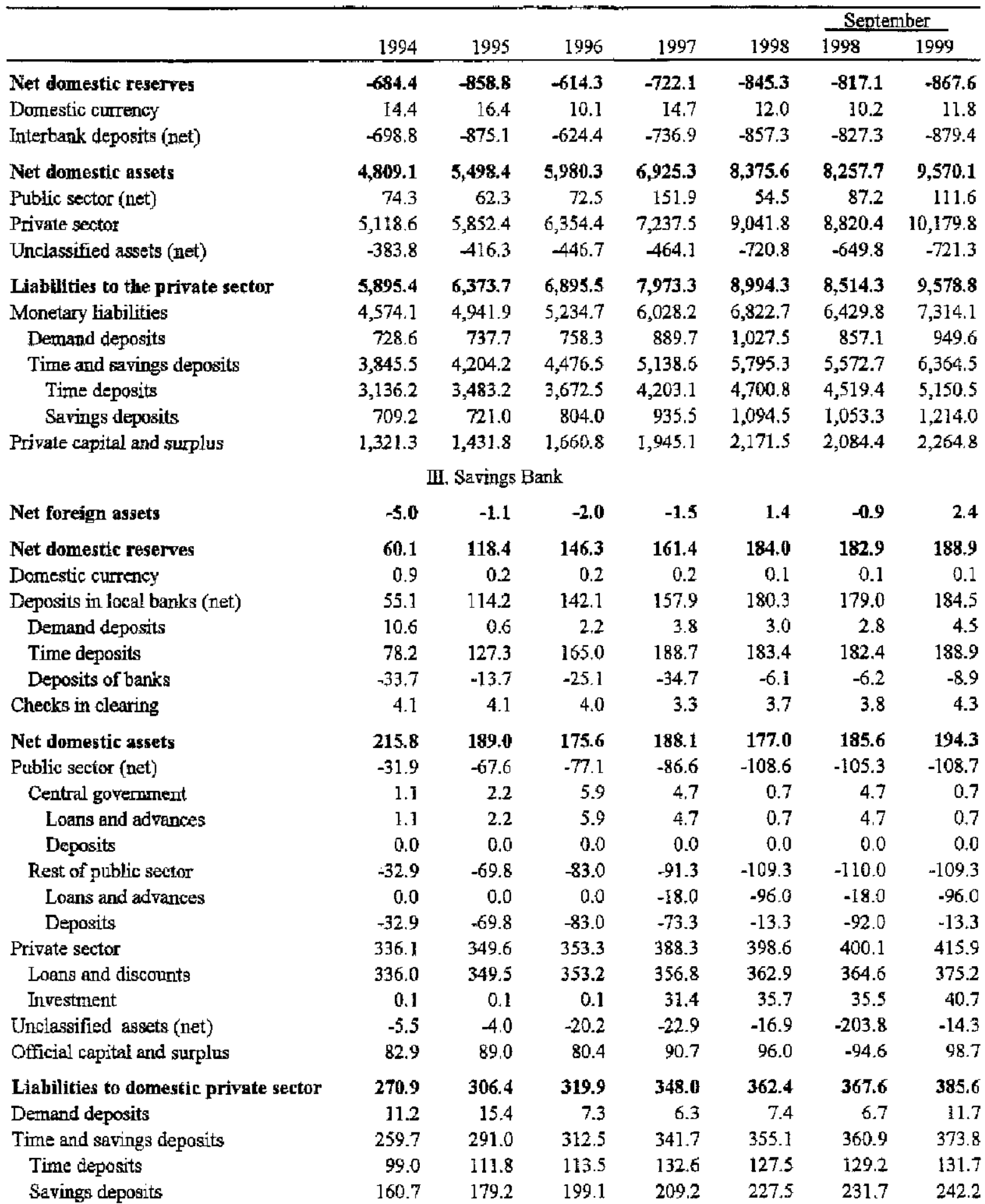


Table 25. Panama: Accounts of the Banking System 1/ (Concluded)

(In millions of balboas; end of period)

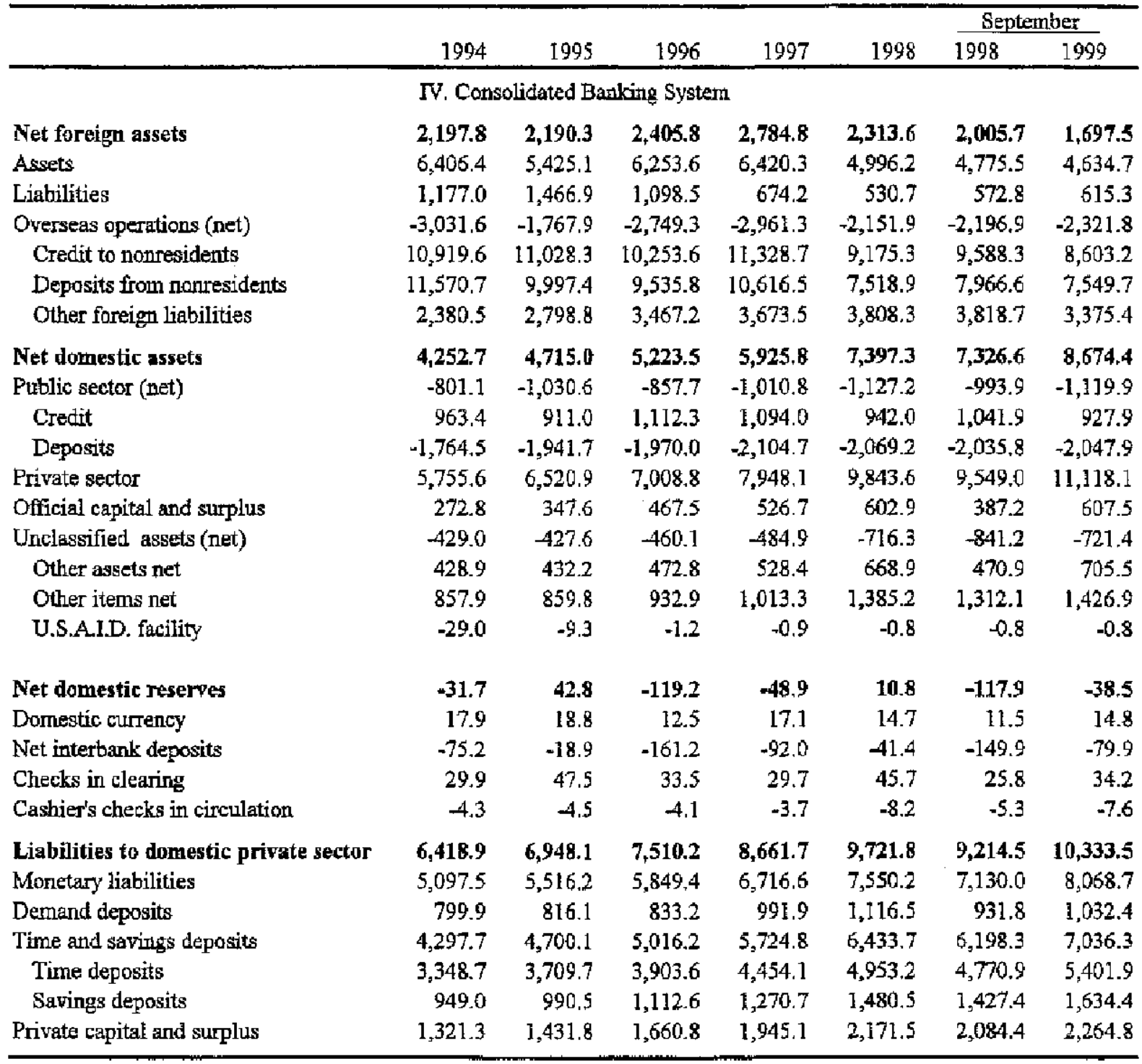

Sources: National Bank of Panama; Superintendency of Banks, Savings Bank; and Fund staff estimates.

1/ Excludes operations of international banks which are licensed to perform only offshore operations, but includes the offshore operations of banks licensed to perform both domestic and offshore operations.

2/ Net Foreign reserves less long term liabilities.

3/ Foreign assets minus short term liabilities.

4/ Not included in BNP accounts since 1987, but is included in the central government accounts.

5/ Excluding the use of Fund credit. 
Table 26. Panama: Summary Operations of the Banking System

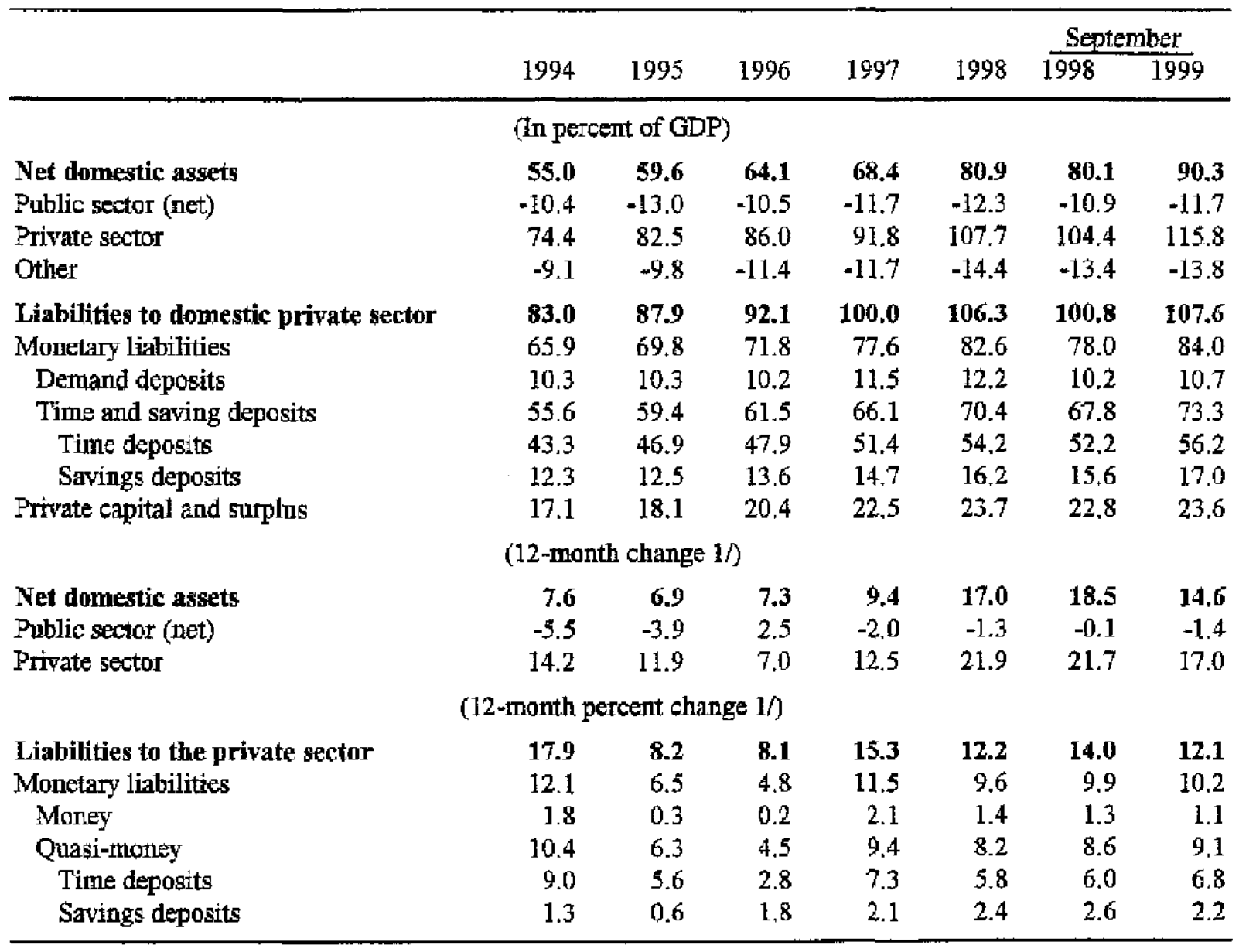

Sources: National Bank of Panama; Superintendency of Banks; Savings Bank; and Furd staff estimates.

1/ In relation to liabilities to the private sector at the beginning of the period. 
Table 27. Panama: Private Sector Deposits

\begin{tabular}{|c|c|c|c|c|c|c|c|}
\hline & \multirow[b]{2}{*}{1994} & \multirow[b]{2}{*}{1995} & \multirow[b]{2}{*}{1996} & \multirow[b]{2}{*}{1997} & \multirow[b]{2}{*}{1998} & \multicolumn{2}{|c|}{ September } \\
\hline & & & & & & 1998 & $199 \overline{9}$ \\
\hline \multicolumn{8}{|c|}{ (In millions of balboas; end of period) } \\
\hline Total private sector deposits & $5,097.5$ & $5,516,2$ & $5,849,4$ & $6,716,6$ & $7,550.2$ & $7,130.0$ & $8,068.7$ \\
\hline Demand & 799.9 & 816.1 & 833.2 & 991.9 & $1,116.5$ & 931.8 & $1,032.4$ \\
\hline National Bank & 60.1 & 63.0 & 67.6 & 95.9 & 81.7 & 67.9 & 71.1 \\
\hline Savings Bank & 11.2 & 15.4 & 7,3 & 6.3 & 7.4 & 6.7 & 11.7 \\
\hline Private banks & 728.6 & 737.7 & 758.3 & 889.7 & $1,027.5$ & 857.1 & 949.6 \\
\hline Time and savings & $4,297.7$ & $4,700.1$ & $5,016.2$ & $5,724.8$ & $6,433.7$ & $6,198.3$ & $7,036.3$ \\
\hline National Bank & 192.5 & 204.9 & 227.2 & 244.4 & 283.4 & 264.6 & 298.0 \\
\hline Savings Bank & 259.7 & 291.0 & 312.5 & 341.7 & 355.1 & 360.9 & 373.8 \\
\hline Private banks & $3,845.5$ & $4,204,2$ & $4,476.5$ & $5,138.6$ & $5,795.3$ & $5,572.7$ & $6,364.5$ \\
\hline Time deposits & $3,348.7$ & $3,709.7$ & $3,903,6$ & $4,454.1$ & $4,953,2$ & $4,770,9$ & $5,401.9$ \\
\hline National Bank & 113.4 & 114,6 & 117.7 & 118,4 & 124.8 & 122.3 & 119.7 \\
\hline Savings Bank & 99.0 & 111.8 & 113.5 & 132.6 & 127.5 & 129.2 & 131.7 \\
\hline Private banks & $3,136.2$ & $3,483.2$ & $3,672.5$ & $4,203.1$ & $4,700.8$ & $4,519.4$ & $5,150.5$ \\
\hline Savings deposits & 949.0 & 990.5 & 1,112.6 & $1,270.7$ & $1,480.5$ & $1,427.4$ & $1,634.4$ \\
\hline National Bank & 79.1 & 90.3 & 109.5 & 126.0 & 158.6 & 142.3 & 178.3 \\
\hline Savings Bank & 160.7 & 179.2 & 199.1 & 209.2 & 227.5 & 231.7 & 242.2 \\
\hline Private banks & 709.2 & 721.0 & 804.0 & 935.5 & $1,094,5$ & $1,053,3$ & $1,214.0$ \\
\hline \multicolumn{8}{|c|}{ (Percentage change over previous 12-month period) } \\
\hline Total private sector deposits & 14.9 & 8.2 & 6.0 & 14.8 & 12.4 & 12.6 & 13,2 \\
\hline Demand & 13.6 & 2.0 & 2.1 & 19.0 & 12.6 & 12.6 & 10.8 \\
\hline Time and savings & 15.1 & 9.4 & 6.7 & 14.1 & 12.4 & 12.6 & 13.5 \\
\hline Time & 17.2 & 10.8 & 5.2 & 14,1 & 11.2 & 11.3 & 13.2 \\
\hline Savings & 8.3 & 4.4 & 12.3 & 14.2 & 16.5 & 17.1 & 14.5 \\
\hline
\end{tabular}

Sources: National Bank of Panama; Superintendency of Banks; Savings Bank; and Fund staff estimates. 


\section{Table 28. Panama: Offshore Operations of Private Banks 1/}

(In billions of U.S. dollars; end of the period)

\begin{tabular}{|c|c|c|c|c|c|c|c|}
\hline & \multicolumn{4}{|c|}{ December } & \multirow[b]{2}{*}{1998} & \multicolumn{2}{|c|}{ September } \\
\hline & 1994 & 1995 & 1996 & 1997 & & 1998 & 1999 \\
\hline Foreign assets & 22,8 & 22.7 & 21.6 & 22.9 & 20.9 & 21.7 & 19.2 \\
\hline Foreign reserves & 8.4 & 7.0 & 7,3 & 7.7 & 6.1 & 6.1 & 5.8 \\
\hline General license banks & 5.7 & 4.6 & 5.2 & 5.3 & 4.0 & 3.7 & 3.7 \\
\hline Oftshore banks $2 /$ & 2.7 & 2,4 & 2.0 & 2.4 & 2.1 & 2.4 & 2.1 \\
\hline Credit to nonresidents & 14.4 & 15.6 & 14.3 & 15.2 & 14.8 & 15.6 & 13.4 \\
\hline General license banks & 10.9 & 11.0 & 10.3 & 11.3 & 9.2 & 9.6 & 8.6 \\
\hline Offshore banks & 3.5 & 4.6 & 4.0 & 3.9 & 5.6 & 6.0 & 4.8 \\
\hline Foreign liabilities & 20.8 & 20.7 & 19.9 & 20.9 & 19.5 & 20.7 & 18.1 \\
\hline Short-term liabilities & 1.1 & 1.3 & 1.1 & 0.7 & 0.7 & 0.7 & 0.5 \\
\hline General license banks & 0.9 & 1. 1 & 1.0 & 0.5 & 0.4 & 0.4 & 0.5 \\
\hline Offishore banks & 0.3 & 0.2 & 0.1 & 0.2 & 0.3 & 0.2 & 0.0 \\
\hline Deposits from nonresidents & 17.2 & 16.5 & 15.3 & 16.5 & 14.7 & 15.9 & 13.7 \\
\hline General license banks & 11.6 & 10.0 & 9.5 & 10.6 & 7.5 & 8.0 & 7.5 \\
\hline Banks & 8.6 & 6.6 & 6.3 & 7.5 & 5,5 & 5.7 & 5.4 \\
\hline Nonbanks & 3.0 & 3.4 & 3.3 & 3.1 & 2,0 & 2.2 & 2.1 \\
\hline Offshore banks & 5.7 & 6.6 & 5.8 & 5.9 & 7.1 & 7.9 & 6.2 \\
\hline Other liabilities & 2.4 & 2.8 & 3.5 & 3.7 & 4.2 & 4.1 & 3.9 \\
\hline General license banks & 2.4 & 2.8 & 3.5 & 3.7 & 3.8 & 3.8 & 3.4 \\
\hline Offshore banks & 0.0 & 0.0 & 0.0 & 0.1 & 0.4 & 0.3 & 0.5 \\
\hline
\end{tabular}

Sources: Superintendency of Banks; and Fund staff estimates.

1/ Includes offshore operations of international license banks.

2/ Refers to international license banks. 
Table 29. Panama: Interest Rate Structure

(Simple period average)

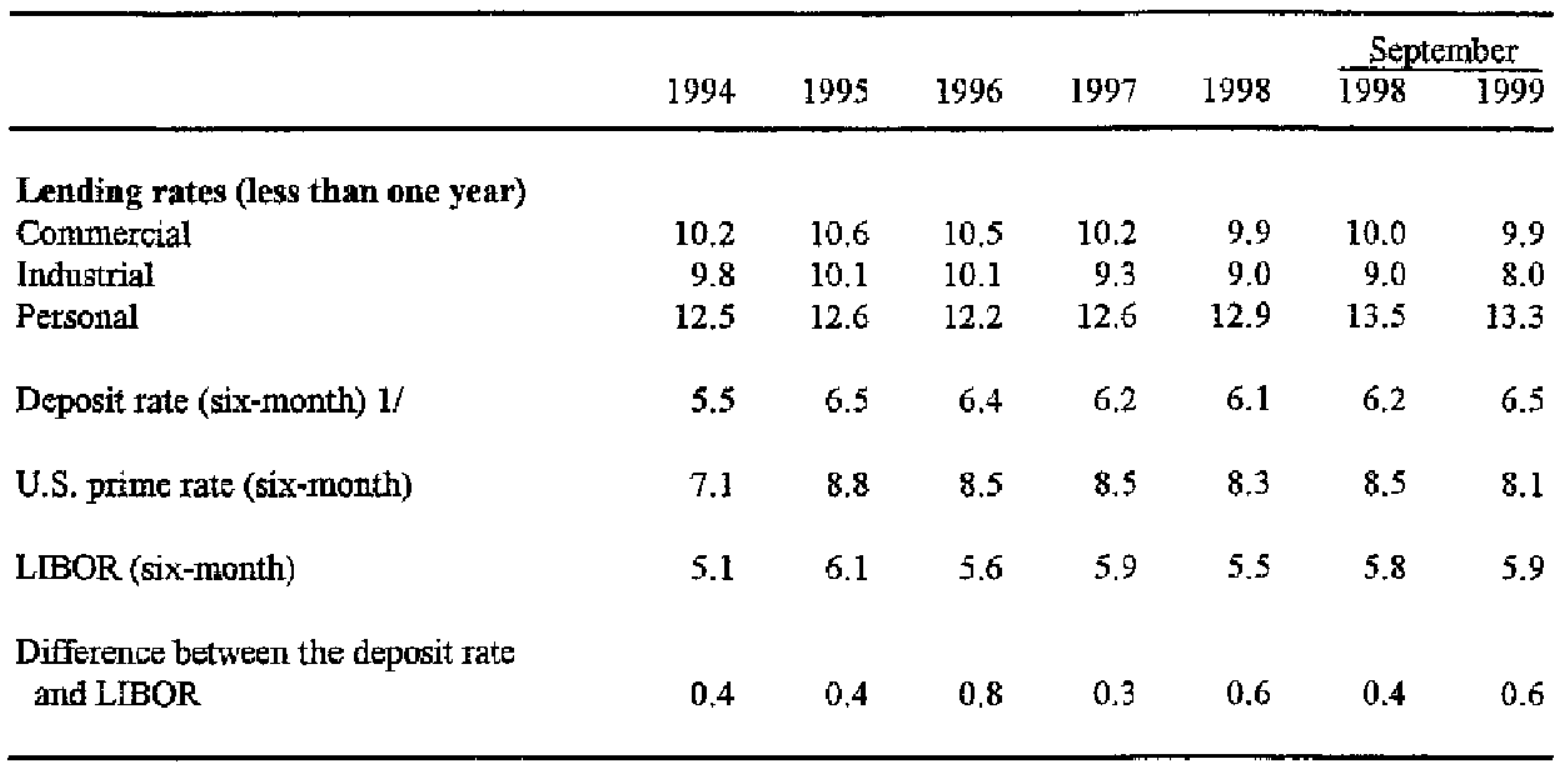

Sources: National Banking Commission; International Financial Statistics; and Fund staff estimates.

1/ Domestic deposit rate. 
Table 30. Panama: Banking System Credit to the Domestic

Private Sector by Economic Activity

\begin{tabular}{|c|c|c|c|c|c|c|c|}
\hline & \multirow[b]{2}{*}{ I994 } & \multirow[b]{2}{*}{1995} & \multirow[b]{2}{*}{1996} & \multirow[b]{2}{*}{1997} & \multirow[b]{2}{*}{1998} & \multicolumn{2}{|c|}{ September } \\
\hline & & & & & & 1998 & 1999 \\
\hline \multicolumn{8}{|c|}{ (In millions of baiboas; end of period) } \\
\hline Total & $5,420.0$ & $6,121.0$ & $6,474.0$ & $7,212,0$ & $8,784.0$ & $8,572,0$ & $9,879.0$ \\
\hline Commerce & $2,394.0$ & $2,671.0$ & $2,568,0$ & $2,896,0$ & $3,426,0$ & $3,538.0$ & $3,769.0$ \\
\hline Housing & $1,334,0$ & $1,493,0$ & $1,649.0$ & $1,844.0$ & $2,124,0$ & $2,045.0$ & $2,313.0$ \\
\hline Other construction & 150,0 & 187,0 & 297.0 & 278.0 & 230.0 & 248.0 & 247.0 \\
\hline Industry & 285.0 & 367.0 & 371.0 & 415.0 & 463.0 & 457.0 & 468.0 \\
\hline Personal consumption & 748.0 & 849.0 & 948.0 & $1,097.0$ & $1,541.0$ & $1,380.0$ & $1,935.0$ \\
\hline Financial and insurance enterprises & 215.0 & 237.0 & 300.0 & 362.0 & 687.0 & 589.0 & 829.0 \\
\hline Agriculture & 124.0 & 130.0 & 137.0 & 129.0 & 129.0 & 130.0 & 120.0 \\
\hline Livestock & 144.0 & 161.0 & 155.0 & 150,0 & 139.0 & 141.0 & 153.0 \\
\hline Fishing & 25.0 & 26.0 & 28.0 & 27,0 & 32.0 & 32.0 & 32.0 \\
\hline Other & 0.0 & 0,0 & 21.0 & 140 & 13.0 & 12.0 & 13.0 \\
\hline \multicolumn{8}{|c|}{ (12-month percentage change) } \\
\hline Total & 14.8 & 12.9 & 5.8 & 11.4 & 21.8 & 21.6 & 15.2 \\
\hline Commerce & 14.8 & 11.6 & -3.9 & 12.8 & 18.3 & 22.9 & 6.5 \\
\hline Housing & 12.8 & 11.9 & 10.4 & 11.8 & 15.2 & 13.8 & 13.1 \\
\hline Other construction & 20.0 & 24.7 & 58.8 & -6.4 & -17.3 & -15.9 & -0.4 \\
\hline Industry & 8.7 & 28.3 & 1.1 & 11.9 & 11.6 & 12.3 & 2.4 \\
\hline Personal consumption & 16,9 & 13.5 & 11.7 & 15.7 & 40.5 & 33.6 & 40.2 \\
\hline Financial and insurance enterprises & 16.8 & 10.2 & 26.6 & 20.7 & 89.8 & 89.4 & 40.7 \\
\hline Agriculture & 14.8 & 4.8 & 5.4 & -5.8 & 0.0 & -3.7 & -7.7 \\
\hline Livestock & 23.1 & 11.8 & -3.7 & -3.2 & -7.3 & -6.0 & 8.5 \\
\hline Fishing & 47.1 & 4.0 & 7.7 & -3.6 & 18.5 & 10.3 & 0.0 \\
\hline \multicolumn{8}{|c|}{ (In percent of total credit) } \\
\hline Total & 100,0 & 100 & 100,0 & 100.0 & 100.0 & 100.0 & 100.0 \\
\hline Commerce & 44.2 & 43.6 & 39.7 & 40.2 & 39.0 & 41.3 & 38.2 \\
\hline Housing & 24.6 & 24.4 & 25.5 & 25.6 & 24.2 & 23.9 & 23.4 \\
\hline Other construction & 2.8 & 3.1 & 4.6 & 3.9 & 2.6 & 2.9 & 2.5 \\
\hline Industry & 5.3 & 6.0 & 5.7 & 5.8 & 5.3 & 5.3 & 4.7 \\
\hline Personal consumption & 13.8 & 13.9 & 14.6 & 15.2 & 17.5 & 16.1 & 19.6 \\
\hline Financial and insurance enterpises & 4.0 & 3.9 & 4,6 & 5.0 & 7.8 & 6.9 & 8.4 \\
\hline Agriculture & 2.3 & 2.1 & 2.1 & 1.8 & 1.5 & 1.5 & 1.2 \\
\hline Livestock & 2.7 & 2.6 & 2.4 & 2.1 & 1.6 & 1.6 & 1.5 \\
\hline Fishing & 0.5 & 0.4 & 0.4 & 0.4 & 0.4 & 0.4 & 0.3 \\
\hline
\end{tabular}

Sources: Superintendency of Banks; and Fund staff estimates. 
Table 31. Panama: Public Sector Banks--Operating

Revenue and Expenditure 1/

(In millions of U.S, dollars)

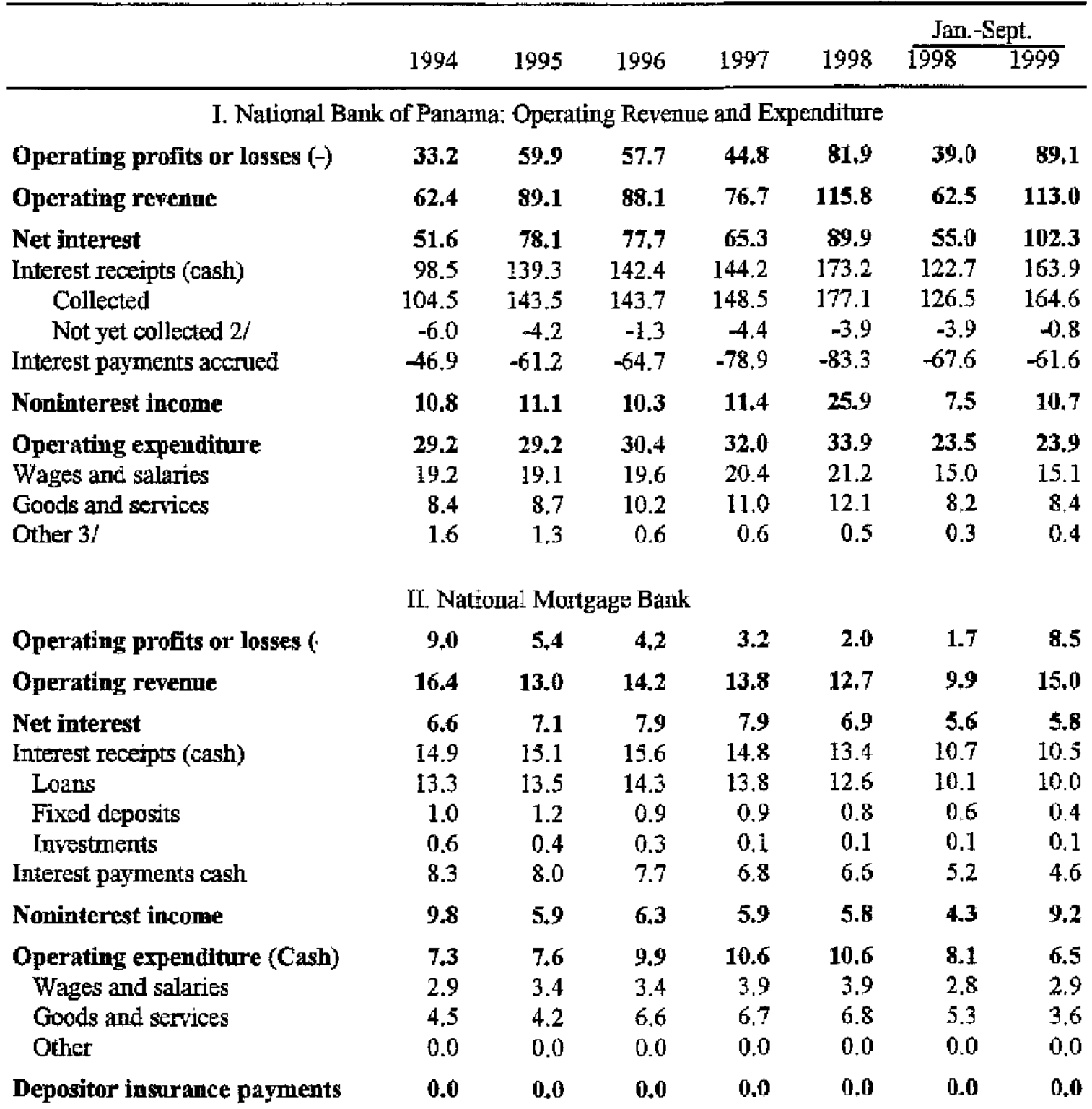


Table 31. Panama: Public Sector Banks--Operating Revenue and Expenditure (Concluded)

(In millions of balboas)

\begin{tabular}{|c|c|c|c|c|c|c|c|}
\hline & \multirow[b]{2}{*}{1994} & \multirow[b]{2}{*}{1995} & \multirow[b]{2}{*}{1996} & \multirow[b]{2}{*}{1997} & \multirow[b]{2}{*}{1998} & \multicolumn{2}{|c|}{ Jan.-Sept. } \\
\hline & & & & & & 1998 & 1999 \\
\hline \multicolumn{8}{|c|}{ III. Agricultural Development Bank } \\
\hline Operating profits or losses (-) & -2.5 & -3.3 & -3.0 & -3.8 & -5.2 & $-3,6$ & -4.1 \\
\hline Operating revenue & 3.2 & 2.8 & 2.1 & 1.4 & 0.0 & 0.7 & -0.2 \\
\hline Net interest & 2.9 & 2.5 & 1.8 & 0.7 & -0.6 & 0.3 & -0.6 \\
\hline Interest receipts (cash)-loans & 7.9 & 6.7 & 5.9 & 4,8 & 5.3 & 4.9 & 5.7 \\
\hline Interest payments accrued 2/ & 4.9 & 4.2 & 4,1 & 4.1 & 5.9 & 4.6 & 6.3 \\
\hline Interest payments cash & 4.6 & 3.9 & 3.8 & 3.6 & 3.3 & 2.4 & 2.3 \\
\hline Noninterest income & 0.3 & 0.3 & 0.3 & 0.7 & 0.6 & 0.4 & 0.4 \\
\hline Operating expenditure (Cash) & 5.7 & 6.1 & 5.1 & 5.2 & 5.2 & 4.4 & 3.9 \\
\hline Wages and salaries & 4.1 & 4.5 & 3.6 & 3.7 & 3.7 & 3.1 & 2.8 \\
\hline Goods and services & 0.9 & 0.9 & 0.9 & 0.9 & 0.9 & 0.7 & 0.6 \\
\hline Other & 0.7 & 0.7 & 0.6 & 0.6 & 0.6 & 0.5 & 0.5 \\
\hline
\end{tabular}

Sources: National Bank of Panama; National Mortgage Bank and Agricultural Development Bank.

1/ Not including the Savings Bank as final accounts for end 1998 and interim accounts for September 1999 were not available.

2/ Difference of interest receivable at the beginning and at the end of the period.

3/ Includes exchange profit of US\$0.5 million in 1996. 


\section{Table 32. Panama: Public Sector Banks--Portfolio in Arrears 1/}

(In millions of baltoas; except where otherwise indicated)

\begin{tabular}{|c|c|c|c|c|c|c|c|}
\hline & \multirow[b]{2}{*}{1994} & \multirow[b]{2}{*}{1995} & \multirow[b]{2}{*}{1996} & \multirow[b]{2}{*}{1997} & \multirow[b]{2}{*}{1998} & \multicolumn{2}{|c|}{ September } \\
\hline & & & & & & 1998 & 1999 \\
\hline \multicolumn{8}{|c|}{ I. National Bank of Panama } \\
\hline Total private sector portfolio 1 f & 262.2 & 276.9 & 269.6 & 260.6 & 327.6 & 276.8 & 472.7 \\
\hline Commeroe & 39.7 & 39.1 & 36.8 & 43.4 & 45.5 & 45.3 & 51.2 \\
\hline Agriculture and forestry & 76,6 & 78.0 & 79.6 & 81.7 & 83.9 & 85.0 & 96.4 \\
\hline Perscnal loans & 91.5 & 99.5 & 92.6 & 75.9 & 184.7 & 135.8 & 299.0 \\
\hline Others & 54.4 & 60.2 & 59.6 & 59.6 & 13.4 & 10,6 & 26.1 \\
\hline Total arrears & 12.7 & 10.7 & 11.5 & 11.1 & 10.7 & 10.0 & 12.1 \\
\hline Commerse & 6.2 & 4.3 & 4.1 & 3.3 & 2.6 & 2.8 & 2.6 \\
\hline Agriculture and forestry & 3.5 & 3.7 & 4.4 & 4.7 & 5.0 & 4.0 & 5.5 \\
\hline Parscinal loans & 0.8 & $\$ .0$ & 0.9 & 1.1 & 1.7 & 1.8 & 2.5 \\
\hline Others & 2.1 & 1.7 & 2.1 & 2.0 & 1.4 & 1.4 & 1.5 \\
\hline Share of portfolio in arrears (in percent) & 4.8 & 3.9 & 4.3 & 4.3 & $\mathbf{3 . 3}$ & 3.6 & 2.6 \\
\hline Commerce & 15.6 & 10.9 & 11.1 & 7.6 & 5.6 & 6.1 & 5.2 \\
\hline Agriculture and forestry & 4.6 & 4,8 & 5.5 & 5.8 & 6.0 & 4.7 & 5,7 \\
\hline Personal loans & 0.9 & 1.0 & 1.0 & 1.5 & 0.9 & 1.3 & 0.8 \\
\hline Others & 3.9 & 2.8 & 3.6 & 3.4 & 10.8 & 12.9 & 5.8 \\
\hline Loans with payments overdure & 12.7 & 10.7 & 11.5 & 11.1 & 10.7 & 10.0 & 12.1 \\
\hline Between 30 and 90 days & 4.6 & 3.8 & 5.1 & 4.8 & 4.2 & 3.7 & 6.7 \\
\hline Over 90 days & 8.1 & 6.9 & 6.4 & 6.4 & 6.4 & 6.3 & 5.5 \\
\hline Share of payments overdue (im percent) & 100.0 & 100.0 & 100.0 & 100.0 & 100,0 & 100.0 & 100.0 \\
\hline Between 30 and 90 days & 36.2 & 35.2 & 44.6 & 42.8 & 39.7 & 36.9 & $\$ 5.1$ \\
\hline Over 90 days & 63.8 & 64.8 & 55,4 & 57.2 & 60.3 & 63.1 & 44.9 \\
\hline \multicolumn{8}{|c|}{ IL. National Housing Bank $\mathbf{3}$ / } \\
\hline Totall portfolio & 166.6 & 174.4 & 188.5 & 195.4 & 204.7 & 206.0 & 200.2 \\
\hline Total arrears & 13,6 & 42.2 & 45.6 & 8.1 & 16.0 & 18.5 & 23.2 \\
\hline Share in arrears (in percent) & 8.2 & 24.2 & 24.2 & 4.1 & 7.8 & 9.6 & 11.6 \\
\hline \multicolumn{8}{|c|}{ III AgriguItural Developrnent Bank 4/ } \\
\hline Total portfolio & 81.4 & 76.6 & 74.2 & 76.1 & 83.9 & 82.1 & 86.3 \\
\hline Loans overdue & 21.6 & 20.4 & 20.7 & 21.2 & 20t.6 & 19.2 & 25.9 \\
\hline \multicolumn{8}{|l|}{ Loans overutue as a share of total } \\
\hline portfolio (in percent) & 26.5 & 26.7 & 27.9 & 27.9 & 24.5 & 23.3 & 30.0 \\
\hline Loans with payments overdue & 21.6 & 20.4 & 20.7 & 21.2 & 20.6 & 19.2 & 25.9 \\
\hline Between 30 to 90 days & 3.3 & 0.7 & 1.0 & 1.1 & 1.2 & 1.4 & 3.3 \\
\hline More than 90 days & 18.3 & 19.7 & 19.7 & 20.2 & 19.4 & 17.8 & 22.6 \\
\hline Tetal with paynents overdne (in percent) & 100.0 & 100.0 & 100.0 & 100.0 & 100.0 & $\mathbf{1 0 0 . 0}$ & 100.0 \\
\hline Between 30 to 90 days & 15.5 & 3.4 & 4.9 & 5.0 & 5.8 & 7.1 & 12.8 \\
\hline More than 90 days & 84.5 & 96.6 & 95.1 & 95.0 & 94.2 & 929 & 87.2 \\
\hline
\end{tabular}

Sources: Nationai Bank of Panama; National Mortgage Bank and the Agricultural Development Bank.

V Does not include the Savings Bank as data for end 1998 and September 1999 were not swailable.

2/ From 1996 refers to loans overdue by more than 91 days.

3/ This portfolio includes bad loans related to projects financed through the Ministry of

Housing aimed at alloviating the problen of housing for lower inoome families.

4/ From 1993 the portfolio excludes bad loans. 
Table 33. Panama: Balance of Payments

(th millions of balbuas)

\begin{tabular}{|c|c|c|c|c|c|c|c|c|c|c|c|c|c|c|c|c|}
\hline & \multicolumn{3}{|c|}{1994} & \multicolumn{3}{|c|}{1995} & \multicolumn{3}{|c|}{1996} & \multicolumn{3}{|c|}{1997} & \multicolumn{3}{|c|}{1998} & \\
\hline & Credit & Debit & Balance & Crexit & Debit & Balarce & Credit & Debit & Bálánce & Credit & Debit & Balanee & Credit & Dubil & Balance & \\
\hline Current acco unt & $8,840.2$ & $\$, 824.4$ & 15.8 & $9,437.0$ & $9,806.1$ & -369.1 & B,968.2 & $9,270.1$ & -301.9 & $2,928.6$ & $10,524.15$ & -603.5 & $9,942.8$ & $11,154,5$ & $-1,211.7$ & \\
\hline Goods and services & $7,448.5$ & 7,3592 & 89.3 & $7,610,4$ & $7,767.7$ & -157.3 & $7,381.2$ & $7,500.7$ & -119.5 & $8,303,8$ & $8,646.3$ & -342.5 & $8,022.6$ & $8,868.8$ & -846.2 & \\
\hline Goods & $6,044.8$ & $6,294,9$ & -250.1 & $6,091.0$ & $6,679.9$ & -588.9 & $5,822.9$ & $6,467.0$ & -644.1 & $6,655,4$ & $7,355.7$ & -700.3 & $6,325.2$ & $7,696.3$ & $-1,371,1$ & \\
\hline Nonpetroleum exports (f,o.b.) & $5,937.4$ & 0.0 & $5,937.4$ & $5,945.2$ & 0.0 & $5,945.2$ & $5,655.5$ & 0.0 & $5,655.5$ & $6,494,2$ & 0.0 & $6,494.2$ & 6196.3 & 0,0 & $6,196,3$ & \\
\hline Petroleum trade (f,a,b.) & 107.4 & 282.2 & -174.8 & 145.8 & 328.6 & -182.8 & 167.4 & 412.4 & .245 .0 & 961.2 & 395.6 & $-234,4$ & 128.9 & 291.4 & -162.5 & \\
\hline Notrpetroleum imports (f,o.b.) & 0.0 & $6,012.7$ & $-6,012.7$ & 0,0 & $6,351.3$ & $-6,351.3$ & 0.0 & $6,054.6$ & $-6,054,6$ & 0.0 & $6,960.1$ & $-6,960.1$ & 0.0 & 74045 & $-7,404.9$ & \\
\hline Services & $1,403.7$ & $1,064,3$ & 339.4 & $1,519,4$ & $1,087,8$ & 431.6 & $1,558.3$ & $1,033.7$ & 524.6 & $1,648,4$ & $1,290.6$ & 357.8 & $1,697.4$ & $1,372.5$ & 524.9 & \\
\hline Transportation & 704.5 & 750.2 & -45.7 & 783.7 & 744.8 & 38.9 & 777.6 & 677.2 & 100.4 & E34.l & 786.3 & 47,8 & 880,3 & 6.54 .2 & 226.1 & \\
\hline \multicolumn{17}{|l|}{ of which: } \\
\hline ZLC & 0.0 & 459.3 & -459.3 & $0.0^{\circ}$ & 451.6 & $-4 \$ 1.6$ & 0.0 & 971.2 & -371.2 & 0.0 & 443.8 & $-443,8$ & 0.0 & 277.9 & -277.9 & \\
\hline Travei & 261.6 & 122.6 & 139.0 & 309.4 & 120.7 & 188.7 & 359.0 & 140.3 & 219.6 & 374.2 & 163.8 & 210,4 & 378.8 & 176.2 & 202.6 & \\
\hline Government & 2313 & 33.9 & 197.4 & 221.7 & 38.6 & 189.1 & 202.3 & 33.5 & 168.8 & 206.1 & 46.2 & 159.9 & 134.3 & 43.1 & 91.2 & \\
\hline Other services & 206.3 & 157.6 & 48.7 & 204.6 & 183.7 & 20.9 & 218,5 & 182.7 & 35.8 & 234.0 & 294.3 & .60 .3 & 304.0 & 299.0 & 5.0 & \\
\hline Income & $1,202,9$ & $1,425,3$ & -222.4 & $1,644.1$ & $2,008.5$ & -364.4 & 1,422.1 & $1,739.3$ & -317.1 & $1,436.0$ & $1,847.6$ & -411.6 & $1,725.2$ & $2,249.7$ & -524.5 & 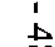 \\
\hline 1 tabor iteome & 94.9 & 0.0 & 94,9 & 95.7 & 0.0 & 95.7 & 70.4 & 00 & 50.4 & 48.1 & 0.0 & 48.1 & 42.3 & 0.0 & 42.3 & $\infty$ \\
\hline Investurient income & $1,108,0$ & $1,425.3$ & -317.3 & $1,548.4$ & $2,008.5$ & -460.1 & $1,351.7$ & $1,739.2$ & -387.5 & $1,387.9$ & $1,847.6$ & 459.7 & 1682.9 & 2249.7 & -566.8 & \\
\hline Interest on public debt & 0.0 & 354.0 & $-354,0$ & 0.0 & 393.2 & -393.2 & 0.0 & 243.3 & -2433 & 0.0 & 282.3 & .282 .3 & 0.0 & 303.2 & -303.2 & \\
\hline Private sector & $1,108.0$ & 1,0713 & 36.7 & $1,548.4$ & $1,615.3$ & -66.9 & $1,351.7$ & $1,495.9$ & .1442 & $1,387.9$ & $1,565.3$ & -177.4 & $1,682.9$ & 1946.5 & $-263,6$ & \\
\hline дcc & 6.3 & 39.9 & -33.6 & 19.3 & 120.4 & $-101 . t$ & 13.9 & 112.1 & -98.2 & 16.4 & 180.7 & -164.3 & 48.7 & 193.0 & -344.3 & \\
\hline Other & $1,101.7$ & $1,091.4$ & 70.3 & $1, \$ 29,1$ & $1,494.9$ & 34.2 & $1,337,8$ & $1,383,8$ & -460 & $1,371.5$ & $1,384.6$ & $\cdot 13.1$ & 1634.2 & 1753.5 & -119.3 & \\
\hline Current transiers & 188.8 & 39,9 & 148.9 & 182.5 & 29.9 & 152.6 & 164.9 & 30.2 & 134.7 & 180.8 & 30.2 & 159.6 & 195.0 & 36.0 & 159.0 & \\
\hline Official transfers & 82.4 & 15.1 & 57.3 & 77.1 & 9.5 & 67.6 & 59.0 & 10.2 & 48.8 & 71.7 & $\mathrm{I} 0.2$ & 61.5 & 75.9 & 11.0 & 68.9 & \\
\hline Other & 106.4 & 24.8 & 81.6 & 105.4 & 20.4 & 85.0 & 105.9 & 20.0 & 85.9 & 109.1 & 20.0 & B9.I & 115.1 & 25.0 & 90.1 & \\
\hline Paid by the U.S. Dept of Defense & 92.6 & 0.0 & 92.6 & 92.6 & 0.0 & 92.6 & 93.9 & 0.0 & 93.9 & 97.4 & 0.0 & 97.4 & 99.2 & 0.0 & 99.2 & \\
\hline Private transfers & 13.8 & 24.8 & -11.0 & 12.8 & 20.4 & -3.6 & 320 & 20.0 & -8.0 & 11.7 & 20.0 & -8.3 & 15.9 & 25.0 & .9 .1 & \\
\hline Capital and financial accoumt $1 /$ & $5,590.3$ & $5,680.1$ & -89.8 & $3,797,1$ & $3,656.2$ & 140.9 & $3,510.3$ & $2,755,0$ & 755.3 & $6,530.3$ & $5,407.5$ & $1,122.8$ & $7,838.4$ & $6,655.3$ & $1,183,1$ & \\
\hline Capital account & 0.0 & 0.0 & 0.0 & 0.0 & 0.0 & 0.0 & 0.0 & u.0 & 0.4 & 39.6 & 0.6 & 70.6 & 56.7 & 0.0 & 56.7 & \\
\hline Official expital transfers & 0.0 & 0.0 & 0,0 & 0,0 & 0.0 & 0.0 & 0.0 & 0.6 & 0.0 & 70.6 & 0,0 & 70.6 & 56.7 & 0.0 & 56.7 & \\
\hline Private capital transfers & 0.0 & 0.0 & 0.0 & 0.0 & 0.0. & 0.0 & 0.0 & 0.0 & 0.0 & 0.0 & 0.0 & 0.6 & 0.0 & 0.0 & 0,0 & $=$ \\
\hline Financlal gceount & $5,590.3$ & $5,680.1$ & -89.8 & $3,797.1$ & $3,656.2$ & $1+10.9$ & $3,510.3$ & $2,755.6$ & 755.3 & $6,459.7$ & $5,407.5$ & $1,052.2$ & $7,781.7$ & $6,655.3$ & $\mathbf{j}, 126.4$ & 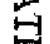 \\
\hline Official capital & 38.3 & 269.1 & -230.8 & 155.0 & 223.7 & -68.7 & 186.4 & 294.8 & -108.4 & $1,454.3$ & $1,958.7$ & $-\$ 04.4$ & 674.3 & 820.5 & .146 .2 & 5 \\
\hline Nonfinareial public sector & 38.3 & 265.1 & $-230,8$ & 99.7 & 223.7 & -124.0 & 186.4 & 294.8 & -108.4 & $1,454.3$ & $1,958.7$ & -504.4 & 674.3 & 816.1 & -141.8 & \\
\hline Medrum and long temt & 38.3 & 269.1 & .230 .8 & 99.7 & 223.7 & -124.0 & 186.4 & 276.8 & -90.4 & $1,440.6$ & $1,222.9$ & 217.7 & 645.6 & 267.4 & 378.2 & \\
\hline Released cellateral & 0.0 & 0.0 & 0.0 & 0.0 & 0.0 & 0.0 & 0.0 & 0.0 & 0.0 & 13.7 & 0.0 & 13.7 & 0.0 & 0,0 & 0,0 & \\
\hline Change in deposits abroad & 0.0 & 0.0 & 0.0 & 0.0 & 0.0 & 0.0 & 0.0 & 18.0 & -18.0 & 0.0 & 550.6 & -550.6 & $28 . ?$ & 56.8 & $-28,1$ & 7 \\
\hline Reiensed collateral & 0.0 & 0.0 & 0.0 & 0.0 & 0.0 & 0.0 & 0.0 & 0.0 & 0.0 & 13.7 & 0,0 & 13.7 & 0.0 & 0.0 & 0.0 & 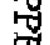 \\
\hline Venezuela oil foan & 0.0 & 11.7 & -11.7 & 0.0 & 12.6 & -12.6 & 0.0 & 12,4 & -12.4 & 0.0 & 0,0 & 0.0 & 0.0 & 0.0 & 0.0 & 2 \\
\hline Mexico oil logn & 0.0 & 0.4 & -0.4 & 0.0 & 0.0 & 0.0 & 0.0 & 0.4 & -0.4 & 0.0 & 0.0 & 0.0 & 0.0 & 0.0 & 0.0 & \\
\hline 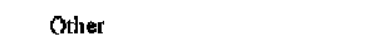 & 0.0 & 0.0 & 0.0 & 0.0 & 0.0 & 0,0 & D. 0 & 0.0 & & 0.0 & 185.2 & -185.2 & 0.0 & 491.9 & -491.9 & \\
\hline
\end{tabular}

CInternational Monetary Fund. Not for Redistribution 
Table 33. Panama: Balance of Payments (Concluded)

(In millions of balboas)

\begin{tabular}{|c|c|c|c|c|c|c|c|c|c|c|c|c|c|c|c|}
\hline & \multicolumn{3}{|c|}{$\left[\log _{4}\right.$} & \multicolumn{3}{|c|}{1995} & \multicolumn{3}{|c|}{1996} & \multicolumn{3}{|c|}{1997} & \multicolumn{3}{|c|}{1998} \\
\hline & Credil & Debit & $\overline{\text { Balance }}$ & Credit & Debit & Balance & Credit & Debit & Balanere & Credit & Debit & Balance & Credit & Debit & $\overline{\text { Balance }}$ \\
\hline Official bank & 0.0 & 0.0 & 0.0 & 55.3 & 0,0 & 55,3 & 0.0 & 0.0 & 0.0 & 0.0 & 0.0 & 0.0 & 0.0 & 4.4 & -4.4 \\
\hline Panama Caral Comınission/Authority & 11.3 & 24.2 & -12.9 & 31.4 & 48.0 & -16.6 & 19.0 & 35.6 & -16.6 & 52.9 & 2.9 & 50.0 & 89.1 & 47.8 & 41.3 \\
\hline Direct investment & 113 & 0.1 & 11.2 & 31.4 & 0.3 & 31.1 & 17.7 & 0.0 & 17.7 & 52.9 & 0.6 & 52.3 & 89.1 & 0.1 & 89.0 \\
\hline Other investment & 0.0 & 24.1 & -24.1 & 0.0 & 47.7 & -47.7 & 1.3 & 35.6 & -34.3 & 0.0 & 2.3 & -2.3 & 0.0 & 47.7 & -47.7 \\
\hline Poivate cópital & $5,540.7$ & $5,3 \mathrm{~B} 6.8$ & 153.9 & $3,610.7$ & $3,384,5$ & 226,2 & $3,304.9$ & $2, \$ 24.6$ & 880.3 & $4,952.5$ & $3,445.9$ & $1,506.6$ & $7,018.3$ & $5,787.0$ & $1,231.3$ \\
\hline Direct investunent & 424,4 & 43.2 & 381.2 & 260.3 & 42.7 & 217.6 & 306,0 & 17.6 & 298,4 & $1,163.1$ & 0,0 & $1,163.1$ & 1,2189 & 101.7 & $1,117.2$ \\
\hline Colon Free Zone & 64.6 & 11.5 & $: 53.1$ & 76.7 & 9.7 & 67.0 & 77.9 & 0.0 & 77,9 & 103,8 & 0,0 & 103.8 & 149.0 & 50.5 & 98.5 \\
\hline Other private seclor & 127.0 & 24.4 & 102.6 & 92.5 & 15.9 & 75.6 & 58.2 & 17.6 & 40.6 & 874.0 & 0.0 & 874,0 & 1029.9 & 0,0 & $1,029.9$ \\
\hline Banks & 232.8 & 73 & 225.5 & 91.1 & 13.1 & 74,0 & 169.9 & 0.0 & 169.9 & 185.3 & 0,0 & 185.3 & 40.0 & 51.2 & -11.2 \\
\hline Portfatio investment & 0.0 & 48.4 & -48.4 & 318.3 & 0.0 & 318.3 & 5709 & 25.8 & 545.1 & 0.0 & $1,0145,4$ & $-1,045.4$ & 533.1 & 99.0 & 434.1 \\
\hline Colon Free Zone & 0.0 & 0.0 & 0.0 & 0.4 & 0.0 & 0.4 & 0.0 & 25.8 & .25 .8 & 0,0 & 0.8 & -0.8 & 0,0 & 11.1 & -11.1 \\
\hline Banks & 0.0 & 48.4 & -48.4 & 317.9 & 0.0 & 317.9 & 570.9 & 0.0 & 570,9 & 0.0 & $1,044.6$ & $-1,044,6$ & 533.1 & 87.9 & 445.2 \\
\hline Other private investment & $5,116.3$ & $5,295.2$ & -178.9 & $3,032.1$ & $3,341,8$ & -309.7 & $2,428.0$ & $2,381.2$ & 46.8 & $3,789,4$ & $2,400,5$ & $1,388.9$ & $5,266.3$ & $5,586,3$ & -320.0 \\
\hline Banks. & $4,915.5$ & $5,254.4$ & -338.9 & $2,874.4$ & $3,173.5$ & -299.1 & $1,926.6$ & $2,210.4$ & -283.8 & $2,944.1$ & $2,171.4$ & 772.7 & 5108.0 & 5268.5 & -160.5 \\
\hline Colon Free Zone & 35.8 & 27.9 & 7.9 & 82.3 & 141.7 & -59.4 & 230.8 & 149.7 & 81.1 & 103.3 & 229.1 & -125.8 & 143.2 & 172.9 & -29.7 \\
\hline Other private sector & 165.0 & 12.9 & 152.1 & 75.4 & 26.6 & $4 B .8$ & 270,6 & 21.1 & 249.5 & 742.0 & 0.0 & 742.0 & 15.1 & 144.9 & -129.8 \\
\hline Foneign position & $3,512.1$ & $3,881.9$ & -369.8 & 952.8 & 919.8 & 33.0 & 180,2 & 22,8 & 157.4 & 211.2 & $2,192.8$ & $-1,981.6$ & & & \\
\hline Errors and omissions & $\ldots$ & $\ldots$ & -130.8 & $\cdots$ & $m$ & -25.0 & 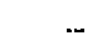 & $\ldots$ & -154.3 & $\cdots$ & $\cdots$ & -376.7 & $\ldots$ & $\cdots$ & $-\$ 94.9$ \\
\hline Overall halance & $\ldots$ & $\cdots$ & -204.8 & $\cdots$ & $\cdots$ & -253.2 & $-\cdots$ & $\cdots$ & 299,1 & $\cdots$ & $\ldots$ & 142.6 & $\ldots$ & $\ldots$ & -223.5 \\
\hline Financing & 494.5 & 289.7 & 204.8 & 369.3 & 116.3 & 253.2 & $3,418.7$ & 3,717.B & -299.1 & 81.5 & 234.1 & .142 .6 & 236.8 & 13.3 & 223.5 \\
\hline Reserves & 14.0 & 147.3 & -133.3 & 12.9 & 116.1 & -103.2 & 75.9 & 295.3 & $-219,4$ & 45.8 & 156.3 & 111.6 & 217.6 & 13.3 & 204.3 \\
\hline \multicolumn{16}{|l|}{ Ne1 foreign assets of the } \\
\hline National Bank of Panama & 0.0 & $146 . I$ & -146.1 & 0.0 & 77.2 & -77.2 & 0.0 & 243.4 & .243 .4 & 0.0 & 130.2 & -330.2 & 176.1 & 0.0 & 176.1 \\
\hline Net ase of Fund credit & 14.0 & 1.2 & 12.8 & 12.9 & 38.9 & -26.0 & 75.9 & 51.9 & 24.0 & 45.8 & $26 . I$ & 19.7 & 41.5 & 13.3 & 28.2 \\
\hline Reschedulting & 480.5 & 0.9 & 480.5 & 77.7 & 0.0 & 77.7 & $3,342.8$ & 0.0 & $3,3.42 .8$ & 35.7 & 0.0 & 35.7 & 19.1 & 0.0 & 19.1 \\
\hline Arrears & 0.0 & 142.4 & .142 .4 & 278.7 & 0.0 & 278.3 & 0.0 & $3,422.5$ & $-3,422.5$ & 0.0 & 67.8 & .67 .8 & 0.1 & 0.0 & 0.1 \\
\hline \multicolumn{16}{|l|}{ Memprandun items: } \\
\hline \multicolumn{16}{|l|}{ Colon Free Zone } \\
\hline Commodity trade & $5,367,6$ & $4,436.0$ & 931.7 & $5,357.2$ & $4,689.5$ & 667.7 & $4,962.5$ & $4,158,3$ & 804.2 & $5,811.1$ & $4,893.5$ & 917.6 & 54610 & 4959.4 & 501.6 \\
\hline Services and income & 37.5 & 635.4 & -557.9 & 51.9 & 595.1 & -543.1 & 45.9 & $\$ 40.3$ & $-494,4$ & 49.4 & 633.8 & -584.3 & 93.0 & $54 \mathrm{~T} .2$ & -584.3 \\
\hline
\end{tabular}

Sources: Office of the Comptroller Genera; Ministry of Planning and Economic Policy; and Fund staff cstimntes.

1/ Excluding errors and omissions

CInternational Monetary Fund. Not for Redistribution 
Table 34, Panama: Summary Balance of Payments

(In millions of balboas, unless otherwise specified)

\begin{tabular}{|c|c|c|c|c|c|c|}
\hline & 1994 & 1995 & 1996 & 1997 & 1998 & $\begin{array}{l}\text { Jan.- } \\
\text { Jane } \\
1999\end{array}$ \\
\hline Current account balance & 15.8 & -369.1 & -301.9 & -603.5 & $-1,211.7$ & -697.5 \\
\hline Trade balance & -250.1 & -588.9 & -644.1 & -700.3 & $-1,371.1$ & -722.7 \\
\hline Exports & 665,0 & 744.4 & 740.6 & 844.3 & 864.2 & 386.8 \\
\hline Non-oil exports & 557.6 & 598.7 & 573.2 & 683.1 & 735.3 & 340.8 \\
\hline Petroleam exports & 107.4 & 145.7 & 167.4 & 161.2 & 128.9 & 45.7 \\
\hline Imports & $-1,858.8$ & $-1,997.4$ & $-2,248.9$ & $-2,462.2$ & $-2,736.9$ & $-1,367.0$ \\
\hline Petroleam imports & -282.2 & -328.6 & -412.4 & -395.6 & -291.4 & $\ldots$ \\
\hline Non-oil imports & $-1,576.6$ & $-1,668.8$ & $-1,836.5$ & $-2,066.6$ & $-2,445.5$ & $\ldots$ \\
\hline Colon Free Zone, net & 943.7 & 664.1 & 864.2 & 917.6 & 501.6 & 257.5 \\
\hline Service balance & 339.4 & 431.6 & 524.6 & 357.8 & 524.9 & 299.8 \\
\hline \multicolumn{7}{|l|}{ Of which: } \\
\hline Colon Free Zone & -459.6 & -451.6 & -371.2 & $-443,8$ & -277.9 & -108.0 \\
\hline Panama Canal Commission/Authority & 515,5 & 585.5 & 598.2 & 633.0 & 689.6 & 352.3 \\
\hline Travel & 139.0 & 188.7 & 219.6 & 210.4 & 202.6 & 87.1 \\
\hline Other servioes, net & 246.1 & 204.0 & 204.6 & 99.6 & 96.2 & 61.4 \\
\hline $\begin{array}{l}\text { Income, net } \\
\text { Of which: }\end{array}$ & -222.4 & -364.4 & -317.1 & -411.6 & -524.5 & -360.1 \\
\hline Public sector interest & -354.0 & -393.2 & -243.3 & -282.3 & -303.2 & -164.5 \\
\hline $\begin{array}{l}\text { Current transfers (net) } \\
\text { Of which: }\end{array}$ & \multicolumn{5}{|c|}{ Of which: } & 85.5 \\
\hline Official transfers (net) & 67.3 & 67.6 & 48.8 & 61.5 & 68.9 & 41,1 \\
\hline Capital and financial account balance $1 /$ & -89.8 & 140.9 & 755.3 & $1,122,8$ & $1,183.2$ & 706.7 \\
\hline Capital account & 0.0 & 0.0 & 0.0 & 70.6 & 56.7 & 0.3 \\
\hline Grants to nonfinancial public sector & 0.0 & 0.0 & 0.0 & 70.6 & 56.7 & 0.3 \\
\hline Financial account balance $1 /$ & -89.8 & 140.9 & 755.3 & $1,052.2$ & $1,126.5$ & 706.4 \\
\hline Public sector (net) & -230.8 & -68.7 & -108.4 & -504.4 & -146.2 & 298.4 \\
\hline Nonfinancial public sector & -230.8 & -124.0 & -108.4 & -504.4 & -141.8 & 301.1 \\
\hline Drawings & 38.3 & 99.7 & 186.4 & $1,440.6$ & 645.7 & 573.6 \\
\hline Amortization & -257.0 & -223.7 & -276.8 & $-1,222.9$ & $-267,4$ & -272.5 \\
\hline Other & -12.1 & 0.0 & -18.0 & $-722,1$ & -520.1 & 0.0 \\
\hline National Bank of Panama & 0.0 & 55.3 & 0.0 & 0.0 & -4.4 & -2.7 \\
\hline Panama Canal Commission/Authority & -12.9 & -16.6 & -16.6 & 50.0 & 41.3 & -3.1 \\
\hline Private capital & 153.9 & 226.2 & 880.3 & $1,506.6$ & $1,231.4$ & 411.1 \\
\hline Errors and omissions & -130.8 & -25.0 & -154.3 & -376.7 & -195.0 & 8.6 \\
\hline Overall balance & -204.8 & -253.2 & 299.1 & 142.6 & -223.5 & 17.8 \\
\hline Financing & 204.8 & 253.2 & -299.1 & -142.6 & 223.5 & -17.8 \\
\hline $\begin{array}{l}\text { Net foreign assets of the National Bank } \\
\text { of Panama (increase -) }\end{array}$ & -146.1 & -77.1 & -243.4 & -130.2 & 176.1 & -22.3 \\
\hline Net use of Fund credit & 12.9 & -26.0 & 24.0 & 19.7 & 28.2 & -11.4 \\
\hline Rescheduling & 480.5 & 77.7 & $3,342.9$ & 35.7 & 19.1 & 15.9 \\
\hline Arrears (net) & -142.4 & 278.6 & $-3,422.6$ & -67.8 & 0.1 & 0.0 \\
\hline \multicolumn{7}{|l|}{ Memorandam item: } \\
\hline Current account (in percent of GDP) & -0.2 & -4.7 & -3.7 & -5.9 & -13.3 & $\ldots$ \\
\hline
\end{tabular}

Sources: Panamanian authorities; and Fund staff estimates. 
Table 35. Panama: Composition of Merchandise Exports

(In millions of balboas, unless otherwise specified)

\begin{tabular}{|c|c|c|c|c|c|}
\hline & 1994 & 1995 & 1996 & 1997 & 1998 \\
\hline Merchandise exports, f.o.b. $1 /$ & 665.0 & 744.4 & 740.6 & 844.3 & 864.2 \\
\hline Petroleum & 107,4 & 145.8 & 167.4 & 173.8 & 128.9 \\
\hline Nonpetroleam exports, I.a.b. $2 /$ & 539.8 & 577.2 & 566.4 & 647.9 & 705.5 \\
\hline $\begin{array}{l}\text { Bananas } \\
\text { Value } \\
\text { Volume (millions of boxes) } \\
\text { Unit value per box }\end{array}$ & $\begin{array}{r}206.6 \\
41.4 \\
5.0\end{array}$ & $\begin{array}{r}190.4 \\
38.0 \\
5.0\end{array}$ & $\begin{array}{r}184.0 \\
34.8 \\
5.3\end{array}$ & $\begin{array}{r}179.8 \\
33.5 \\
5.4\end{array}$ & $\begin{array}{r}138.7 \\
25.5 \\
5.4\end{array}$ \\
\hline $\begin{array}{l}\text { Sugar } \\
\text { Value } \\
\text { Volume (thousands of metric tons) } \\
\text { Unit value per pound }\end{array}$ & $\begin{array}{r}17.1 \\
54.8 \\
0.1\end{array}$ & $\begin{array}{r}18.0 \\
44.2 \\
0.2\end{array}$ & $\begin{array}{r}22.6 \\
46.6 \\
0.2\end{array}$ & $\begin{array}{r}28.7 \\
62.4 \\
0.2\end{array}$ & $\begin{array}{r}25.5 \\
66.2 \\
0.2\end{array}$ \\
\hline $\begin{array}{l}\text { Shrimp } \\
\text { Value } \\
\text { Volume (thousands of metric tons) } \\
\text { Unit value per pound }\end{array}$ & $\begin{array}{r}69.5 \\
7.9 \\
4.0\end{array}$ & $\begin{array}{r}82.8 \\
10.4 \\
3.6\end{array}$ & $\begin{array}{r}74.6 \\
11.0 \\
3.1\end{array}$ & $\begin{array}{r}95.7 \\
12.7 \\
3.4\end{array}$ & $\begin{array}{r}136.7 \\
14.1 \\
4.4\end{array}$ \\
\hline $\begin{array}{l}\text { Coffee } \\
\text { Value } \\
\text { Voilume (thousands of metric tons) } \\
\text { Unit value per pound }\end{array}$ & $\begin{array}{r}14.0 \\
5.3 \\
1.2\end{array}$ & $\begin{array}{r}33.4 \\
10.5 \\
1.4\end{array}$ & $\begin{array}{r}18.8 \\
8.4 \\
1.0\end{array}$ & $\begin{array}{r}22.4 \\
7.5 \\
1.4\end{array}$ & $\begin{array}{r}23.9 \\
8.5 \\
1.3\end{array}$ \\
\hline $\begin{array}{l}\text { Fishmeal } \\
\text { Value } \\
\text { Volume (thousands of metric tons) } \\
\text { Unit value per pound }\end{array}$ & $\begin{array}{r}5.1 \\
19.1 \\
0.1\end{array}$ & $\begin{array}{r}5.0 \\
20.5 \\
0.1\end{array}$ & $\begin{array}{l}2.2 \\
7.1 \\
0.1\end{array}$ & $\begin{array}{r}4.5 \\
11.1 \\
0.2\end{array}$ & $\begin{array}{r}6.6 \\
14.3 \\
0.2\end{array}$ \\
\hline $\begin{array}{l}\text { Other seafood } \\
\text { Value } \\
\text { Volume (thousands of metric tons) } \\
\text { Unit value per pound }\end{array}$ & $\begin{array}{r}18.4 \\
6.2 \\
1.4\end{array}$ & $\begin{array}{r}19.5 \\
5.4 \\
1.7\end{array}$ & $\begin{array}{r}22.1 \\
7.2 \\
1.4\end{array}$ & $\begin{array}{r}36.4 \\
13.4 \\
1.2\end{array}$ & $\begin{array}{r}56.0 \\
19.8 \\
1.3\end{array}$ \\
\hline $\begin{array}{l}\text { Clothing } \\
\text { Value } \\
\text { Volume (metric tons) } \\
\text { Unit value per pound }\end{array}$ & $\begin{array}{r}20.5 \\
0.6 \\
34.2\end{array}$ & $\begin{array}{r}22.2 \\
0.6 \\
37.0\end{array}$ & $\begin{array}{r}20.1 \\
0.6 \\
33.5\end{array}$ & $\begin{array}{r}24.8 \\
0.7 \\
35.4\end{array}$ & $\begin{array}{r}25.6 \\
0.8 \\
32.0\end{array}$ \\
\hline $\begin{array}{l}\text { Other exports } \\
\text { Value }\end{array}$ & 188.6 & 205.9 & 222.0 & 255.6 & 292.5 \\
\hline Balance of payments adjustments for coverage & -19.8 & -21.2 & -26.4 & -13.9 & -26.3 \\
\hline Re-exports & 37.6 & 42.6 & 33.2 & 36.5 & 56.1 \\
\hline
\end{tabular}

Sources: Office of the Comptroller General; and Fund staff estimates.

1/ Including re-exports and balance of payments adjustments.

2/ Excluding re-exports and balance of payments adjustments. 
Table 36. Panama: Petroleum Trade

(In millions of balboas; volumes in millions of barrels; and unit values in balboas per barrel)

\begin{tabular}{|c|c|c|c|c|c|}
\hline & 1994 & 1995 & 1996 & 1997 & 1998 \\
\hline Net imperts & 191.7 & 157.7 & 227.5 & 210.7 & 162.5 \\
\hline $\begin{array}{l}\text { Total imports, f.o.b. } \\
\text { Crude oil }\end{array}$ & 299.1 & 303.5 & 394.9 & 371.9 & 291.4 \\
\hline $\begin{array}{l}\text { Value } \\
\text { From: }\end{array}$ & 118.8 & 135.0 & 289.0 & 262.2 & 188.0 \\
\hline Ecuador & 95.3 & 116.6 & 95.8 & 162.0 & 94.5 \\
\hline Venezuela & 14.9 & 18.4 & 147.0 & 49.2 & 63.3 \\
\hline Mexico & 0.0 & 0.0 & 0.0 & 17.6 & 12.0 \\
\hline Other & 8.6 & 0.0 & 46.2 & 33.4 & 18.2 \\
\hline Refined products & 180.3 & 168.5 & 105.9 & 109.7 & 103.4 \\
\hline Refinery & 180.3 & 168.5 & 105.9 & 109.7 & 103.4 \\
\hline Other & 0.0 & 0.0 & 0.0 & 0.0 & 0.0 \\
\hline Total exports, f.o.b. & 107.4 & 145.8 & 167.4 & 161.2 & 128.9 \\
\hline To other countries & 10.5 & 8.7 & 20.5 & 22.3 & 33.8 \\
\hline Bunker oil for ships & 63.0 & 81.7 & 122.0 & 108.3 & 67.1 \\
\hline Fuel for aircraft & 33.9 & 55.4 & 24.9 & 30.6 & 28.0 \\
\hline \multicolumn{6}{|l|}{ Memorandum items } \\
\hline Volume & 8.5 & 8.8 & 14.7 & 15.0 & 16.6 \\
\hline Unit value & 14.0 & 15.4 & 20.5 & 17.5 & 11.3 \\
\hline
\end{tabular}

Sources: Office of the Comptroller General; Ministry of Commerce and Industry; and Fund staff estimates. 
Table 37. Panama: Nontraditional Exports and Issue of Tax Credit Certificates (In millions of balboas)

\begin{tabular}{|c|c|c|c|c|c|}
\hline & 1994 & 1995 & 1996 & 1997 & $1998 \mathrm{I}$ \\
\hline Nontraditional exports, fo, $b, 2 /$ & 82.2 & 100.8 & 129.5 & 125.6 & 152.7 \\
\hline Food & 1.0 & 3.1 & 2.8 & 11.9 & $\ldots$ \\
\hline Other agricultural products & 23.8 & 35.4 & 46.2 & 51.3 & $\ldots$ \\
\hline Beverages and tobacco & 9.8 & 8.2 & 10.3 & 9.7 & $\ldots$ \\
\hline Metals & 0.0 & 0.0 & 0.0 & 0.0 & $\ldots$ \\
\hline Manufactures & 47.6 & 54.2 & 70.2 & 52.7 & $\ldots$ \\
\hline Issues of tax credit certificates $2 /$ & 12.6 & 13.8 & 19.1 & 18.8 & 24.9 \\
\hline Food & 0.1 & 0.3 & 0.3 & 2.2 & $\ldots$ \\
\hline Agribusiness & & & & & 2.9 \\
\hline Other agricultural products & 5.4 & 5.2 & 8.5 & 7.9 & \\
\hline Agricultural products & & & & & 8.6 \\
\hline Beverages and tobacco & 2.3 & 1.0 & 1.5 & 1.5 & $\ldots$ \\
\hline Sea products & & & & & 5.9 \\
\hline Manufactures & 4.8 & 7.3 & 8.8 & 7.2 & 7.5 \\
\hline $\begin{array}{l}\text { Ratio of tax credit certificates } \\
\text { to nontraditional exports (in percent) }\end{array}$ & 15.3 & 13.7 & 14.8 & 14.9 & 16.3 \\
\hline
\end{tabular}

Sources: Panama Trade Development Institute (IPCE) until 1997; Ministry of Commerce and Industry in 1998; and Fund staff estimates.

1/ The classification of tax credit certificates for nontraditional exports changed in 1998.

2/ Excludes nontraditional exports not benefiting from tax credit certificates. 
Table 38. Panama: Composition of Merchandise Imports, c.i.f.

(Value in millions of balboas; indices: $1988=100$ )

\begin{tabular}{lrrrrrr}
\hline & 1993 & 1994 & 1995 & 1996 & 1997 & 1998 \\
\hline Food & & & & & & \\
Value & 195.3 & 211.9 & 230.9 & 267.0 & 269.9 & 326.3 \\
Volume index & 291.4 & 315.4 & 346.9 & 385.1 & 381.2 & $\ldots$ \\
Unit value index & 72.1 & 72.1 & 71.4 & 74.8 & 76.3 & $\ldots$ \\
Capital goods & & & & & & \\
Value & 514.5 & 541.9 & 596.3 & 679.6 & 762.9 & 891.3 \\
Volume index & 352.4 & 379.8 & 373.0 & 427.1 & 488.8 & $\ldots$ \\
Unit value index & 108.9 & 106.6 & 116.7 & 119.2 & 116.5 & $\ldots$ \\
Petroleum & & & & & & \\
Value & 182.1 & 118.8 & 135.0 & 289.6 & 262.2 & 188.0 \\
Volume index & 182.5 & 125.1 & 129.5 & 216.4 & 220.8 & 244.3 \\
Unit value index & 122.2 & 116.4 & 128.0 & 170.4 & 145.5 & 93.9 \\
Other consumer goods & & & & & & \\
Value & $1,293.4$ & $1,522.8$ & $1,563.4$ & $1,532.8$ & $1,711.4$ & $1,996.1$ \\
Volume index & 233.6 & 405.9 & 386.9 & 386.5 & 423.3 & $\ldots$ \\
Unit value index & 112.1 & 75.8 & 81.1 & 82.2 & 83.2 & $\ldots$ \\
Total value & $2,195.2$ & $2,402.1$ & $2,535.3$ & $2,781.1$ & $3,006.4$ & $3,398.3$ \\
& & & & & & \\
\hline
\end{tabular}

Sources: Office of the Comptroller General; and Fund staff estimates. 
Table 39. Panama: Direction of Trade $1 /$

(In percent)

\begin{tabular}{|c|c|c|c|c|c|}
\hline & 1994 & 1995 & 1996 & 1997 & $\begin{array}{l}\text { Prel. } \\
1998\end{array}$ \\
\hline Exports, f.o.b. & 100.0 & 100.0 & 100.0 & 100.0 & 100.0 \\
\hline Western Hemisphere & 61.0 & 66.6 & 73.3 & 73.6 & 68.2 \\
\hline United States & 37.8 & 41.9 & 47.5 & 45.2 & 40.0 \\
\hline Central America and the Caribbean & 19.2 & 19.6 & 19.3 & 21.2 & 20.7 \\
\hline South America & 2.7 & 3.7 & 4.1 & 3.9 & 3.7 \\
\hline Other & 1.3 & 1.4 & 2.4 & 3.3 & 3.8 \\
\hline Europe & 33.9 & 30.7 & 23.0 & 22.6 & 27.0 \\
\hline Belgium and Luxembourg & 7.0 & 4.8 & 4.2 & 5.4 & 4,3 \\
\hline Germany & 12.8 & 12.4 & 4.5 & 3.1 & 3.4 \\
\hline Italy & 1.8 & 1.1 & 1.0 & 0.7 & 2.7 \\
\hline Sweden & 9.0 & 4.8 & 10.2 & 8.4 & 7.2 \\
\hline Other & 3.3 & 7.6 & 3.1 & 5.0 & 9.4 \\
\hline $\begin{array}{l}\text { Other countries } \\
\text { Of which: }\end{array}$ & 5.1 & 2.7 & 3.7 & 3.8 & 4.8 \\
\hline Exports through the Colon Free Zone & 1.3 & 1.1 & 2.0 & 2.0 & 1.9 \\
\hline Imports, c.i.f. $2 /$ & 100.0 & 100.0 & 100.0 & 100.0 & 100.0 \\
\hline Western Hemisphere & 60.9 & 63.7 & 65.7 & $64.0^{\circ}$ & 63.9 \\
\hline United States & 38.0 & 39.4 & 37.4 & 36.7 & 39.7 \\
\hline Mexico & 2.0 & 3.3 & 3.8 & 4.9 & 4.8 \\
\hline Central America and the Caribbean & 9.1 & 7.6 & 7.1 & 7.4 & 8.0 \\
\hline Costa Rica & 2.8 & 2.9 & 2.7 & 2.9 & 3.0 \\
\hline Trinidad and Tobago & 0.7 & 0.4 & 0.3 & 0.5 & 0.3 \\
\hline Other & 5.6 & 4.3 & 4.1 & 4.0 & 4.7 \\
\hline South America & 11.8 & 13.4 & 17.4 & 15.0 & 11.4 \\
\hline Venezuela & 2.2 & 3.2 & 7.2 & 3.9 & 3.1 \\
\hline Ecuador & 4.4 & 5.3 & 3.7 & 5.6 & 3.2 \\
\hline Brazil & 1.7 & 1.4 & 1.1 & 1.0 & 0.8 \\
\hline Other & 3.5 & 3.5 & 5.4 & 4.5 & 4.3 \\
\hline Europe & 9.6 & 8.8 & 8.3 & 8.8 & 8.4 \\
\hline Germany & 1.8 & 1.9 & 1.7 & 1.3 & 1.3 \\
\hline France & 0.6 & 0.6 & 0.5 & 1.5 & 0.9 \\
\hline Spain & 1.0 & 1.2 & 1.0 & 1.3 & 1.3 \\
\hline Italy & 1.4 & 0.9 & 0.8 & 0.7 & 0.6 \\
\hline Netherlands & 1.8 & 1.3 & 0.9 & 0.6 & 0.7 \\
\hline United Kingdom & 0.6 & 0.6 & 0.6 & 0.8 & 1.2 \\
\hline Other & 2.4 & 2.3 & 2.8 & 2.6 & 2.4 \\
\hline Other countries & 29.5 & 27.5 & 26.0 & 27.2 & 27.7 \\
\hline \multicolumn{6}{|l|}{ Of which: } \\
\hline Imports from the Colon Free Zone & 15.3 & 14.3 & 13.6 & 13.9 & 12.8 \\
\hline
\end{tabular}

Sources: Office of the Comptroller General; and Fund staff estimates.

1/ Based on Customs data.

2/ Excludes sales of bunker oil. 
Table 40. Panama: Net Services Receipts

(In millions of balboas)

\begin{tabular}{|c|c|c|c|c|c|c|}
\hline & 1994 & 1995 & 1996 & 1997 & 1998 & $\begin{array}{r}\text { Jan-June } \\
1999\end{array}$ \\
\hline Total service receipts (net) & 339,4 & 431.6 & 524.6 & 357.8 & 524.9 & 299.8 \\
\hline Transportation & -45.7 & 38.9 & 100.4 & 47.8 & 226.1 & 151.3 \\
\hline Freight and insurance & -710.2 & -703.0 & -622.5 & -731.9 & -595.0 & -377.2 \\
\hline Colon Free Zone & .475 .0 & -468.3 & -385.2 & -460.0 & -293.9 & -109.2 \\
\hline Other & -235.2 & -234.7 & -237.3 & -271.9 & -301.1 & -268.0 \\
\hline Canal receipts, net & 515.0 & 585.5 & 598.2 & 633.0 & 689.6 & 354.8 \\
\hline Other & 149.5 & 156.4 & 124.7 & 146.7 & 131.5 & 173.7 \\
\hline Travel & 139.4 & 188.7 & 219.6 & 210.4 & 202.6 & 87.1 \\
\hline Government & -17.8 & -20.7 & -18.4 & -41.7 & $-26,9$ & $-I 1.2$ \\
\hline \multicolumn{7}{|l|}{ Purchases by the U.S. } \\
\hline Dept. of Defense & 207.2 & 197.3 & 177.7 & 180.9 & 109.1 & 48.2 \\
\hline Other services (net) & 56.3 & 27.4 & 45.3 & -39.6 & 14.0 & 24.4 \\
\hline Total income receipts (net) & -222.4 & -364.4 & -317.1 & -411.6 & -524.5 & -360.1 \\
\hline $\begin{array}{l}\text { Investment income, net } \\
\text { Of which: }\end{array}$ & -317.3 & -460.1 & -387.5 & -459.7 & -566.8 & -377.1 \\
\hline Interest on the public debt & -354.0 & -393.2 & -243.3 & -282.3 & -303.2 & -164.5 \\
\hline Colon Free Zone & -114.0 & -101.1 & -98.2 & -164.3 & -140.7 & -78.0 \\
\hline \multicolumn{7}{|l|}{ Wages paid by the U.S. } \\
\hline Dept. of Defense & 94.9 & 95.7 & 70.4 & 48.1 & 42.3 & 17.0 \\
\hline
\end{tabular}

Sources: Office of the Accountant General; and Fund staff estimates. 
Table 41. Panama: Operations of the Colon Free Zone

(In millions of balboas)

\begin{tabular}{|c|c|c|c|c|c|}
\hline & 1994 & 1995 & 1996 & 1997 & $\begin{array}{c}\text { Prel. } \\
1998\end{array}$ \\
\hline Imports 1 & $4,927.4$ & $5,160.5$ & $4,623.7$ & $5,389.6$ & $5,318.7$ \\
\hline Textiles and clothing & $1,310.7$ & $1,255.2$ & 973.0 & $1,200.0$ & $1,185.7$ \\
\hline Beverages and tobacco & 120.7 & 119.7 & 176.1 & 207.8 & 176.9 \\
\hline Chemical products & 413.1 & 448.7 & 496.1 & 577.7 & 624.2 \\
\hline Instruments & 616.9 & 416.2 & 317.6 & 340.9 & 309.2 \\
\hline Machinery and transport goods & $1,457.2$ & $1,783.7$ & $1,502.4$ & $1,654.8$ & $1,675.6$ \\
\hline Other & $1,008.8$ & $1,137.0$ & $1,158.5$ & $1,408.4$ & $1,347.1$ \\
\hline Exports, f.a.b. $1 /$ & $5,756.7$ & $5,731.4$ & $5,491.7$ & $6,276,0$ & $5,969.3$ \\
\hline Textiles and clothing & $1,478.7$ & $1,339.4$ & $1,177.2$ & $1,384.2$ & $1,271.6$ \\
\hline Beverages and tobacco & 143.1 & 142.2 & 183.1 & 218.0 & 200.1 \\
\hline Chemical products & 595.4 & 644.7 & 675.6 & 770.5 & 829.6 \\
\hline Instruments & 735.3 & 457.7 & 3882 & 373.8 & 347.3 \\
\hline Machinery and transport goods & $1,666.5$ & $1,896.3$ & $1,803.4$ & $1,960.2$ & $1,846.6$ \\
\hline Other & $1,137.7$ & $1,251.1$ & $1,264.2$ & $1,569.3$ & $1,474.1$ \\
\hline \multicolumn{6}{|l|}{ Memorandum item: } \\
\hline $\begin{array}{l}\text { Number of Panamanians employ } \\
\text { in Colon Free Zone 2/ }\end{array}$ & 12,674 & 13,421 & 12,152 & 13,639 & 14,200 \\
\hline
\end{tabular}

Sources: Office of the Comptroller General; Administration of the Colon Free Zone; and Fund staff estimates.

1/ Excludes balance of payments adjustments.

2/ Average for the year. 
Table 42. Panama: Canal Statistics

\begin{tabular}{|c|c|c|c|c|c|c|c|}
\hline \multirow[b]{3}{*}{$\begin{array}{l}\text { Fiscal year } \\
\text { Ended } \\
\text { Sep. } 30 \\
\end{array}$} & \multirow{2}{*}{\multicolumn{4}{|c|}{ Total Traffic }} & \multicolumn{3}{|c|}{ Traffic Assessed Toll on Net Tonnage Basis $/ /$} \\
\hline & & & & & \multirow{2}{*}{$\begin{array}{c}\text { Number } \\
\text { of } \\
\text { Transits } \\
\text { (In thousands) }\end{array}$} & \multirow{2}{*}{$\begin{array}{l}\text { Net Tonnage 2/ } \\
\text { (In millions } \\
\text { of p.c. } \\
\text { net tons) }\end{array}$} & \multirow{2}{*}{$\begin{array}{c}\text { Average Tonnage } \\
\text { per transit } \\
\text { (In thousands of } \\
\text { p.c. net tons) }\end{array}$} \\
\hline & $\begin{array}{c}\text { Number of } \\
\text { Transits } \\
\text { (In thousands) }\end{array}$ & $\begin{array}{c}\text { Tolls } \\
\text { (In millions } \\
\text { of balboas) }\end{array}$ & $\begin{array}{l}\text { Cargo Tonnage } \\
\text { (In millions } \\
\text { of long tons) }\end{array}$ & $\begin{array}{c}\text { Average Toll } \\
\text { (balboa per } \\
\text { kong ton) }\end{array}$ & & & \\
\hline \multicolumn{8}{|c|}{ Total traffic } \\
\hline 1994 & 14.0 & 419.2 & 170.8 & 2.5 & 13.7 & 195.2 & 14.2 \\
\hline 1995 & 15.1 & 462.8 & 190.4 & 2.4 & 14.8 & 216.3 & 14.6 \\
\hline 1996 & 15.2 & 486.7 & 198.5 & 2.5 & 14.9 & 228.3 & 15.3 \\
\hline 1997 & 14.7 & 493.7 & 189.9 & 2.6 & 14.3 & 217.5 & 15.2 \\
\hline 1998 & 14.2 & 545.7 & 192.2 & 2.8 & 14.1 & 222.6 & 15.8 \\
\hline \multicolumn{8}{|c|}{$\begin{array}{l}\text { Of which: } \\
\text { Commercial } \\
\text { Ocean traffic 3/ }\end{array}$} \\
\hline 1994 & 12.2 & 416.8 & 170.5 & 2.4 & 12.3 & 194.3 & 15.8 \\
\hline 1995 & 13.4 & 460.0 & 190.3 & 2.4 & 13.4 & 215.4 & 16.1 \\
\hline 1996 & 13.5 & 483.1 & 198.1 & 2.4 & 13,5 & 226.9 & 16.8 \\
\hline 1997 & 13.0 & 491.6 & 189.8 & 2.6 & 13.0 & 216.9 & 16.7 \\
\hline 1998 & 12.9 & 543.0 & 192.1 & 2.8 & 12.9 & 221.6 & 17.2 \\
\hline
\end{tabular}

Sources: Panama Canal Commission; and Fund staff estimates.

1/ Traffic tolls not assessed on net tonnage basis are assessed on displacement-tonnage basis.

2/ One Panama Canal (p.c) net ton equals 100 cubic feet space. Noncommercial ocean traffic is mainly U.S. Government traffic.

$3 /$ Ocean traffic includes ships of 300 p.c. net tons and over. 
Table 43. Panama: Principal Commodities Shipped Through the Canal

(In thousands of long tons)

\begin{tabular}{|c|c|c|c|c|c|c|c|c|c|c|}
\hline \multirow{2}{*}{$\begin{array}{l}\text { Fiscal Yeas } \\
\text { Ended Septeniber } 30\end{array}$} & \multicolumn{5}{|c|}{ Atlantic to Pacific } & \multicolumn{5}{|c|}{ Pacific to Atlantic } \\
\hline & $\overline{1994}$ & 1995 & 1996 & 1997 & $\overline{1998}$ & $\overline{1994}$ & 1995 & 1996 & 1997 & $\overline{1998}$ \\
\hline Total & 102,595 & 120,845 & 124,205 & 115,547 & 107,257 & 67,943 & 69,458 & 73,861 & 74,233 & 81,040 \\
\hline Agricultural & 31,531 & 43,684 & 41,420 & 34,592 & 34,801 & 13,075 & 12,172 & 13,124 & 12,580 & 12,087 \\
\hline Canned and refrigerated foods & 1,113 & 1,218 & 1,230 & 1,213 & 1,118 & 4,883 & 5,645 & 5,717 & 5,761 & 5,251 \\
\hline Grains & 29,547 & 41,118 & 39,279 & 32,399 & 32,901 & 4,520 & 2,956 & 3,155 & 2,912 & 2,892 \\
\hline Other & 871 & 1,348 & 911 & 980 & 782 & 3,672 & 3,571 & 4,252 & 3,907 & 3,944 \\
\hline Mining products & 21,427 & 21,960 & 22,022 & 20,596 & 18,424 & 19,233 & 21,458 & 23,796 & 25,152 & 24,478 \\
\hline Minerals & 186 & 159 & 130 & 101 & 70 & 5,602 & 5,268 & 6,842 & 7,126 & 6,224 \\
\hline Phosphates and fertilizers & 13,603 & 14,451 & 14,805 & 13,888 & 13,391 & 1,834 & 1,457 & 1,135 & 1,193 & 1,144 \\
\hline Ores and metals & 3,766 & 4,128 & 3,641 & 3,776 & 2,920 & 6,330 & 6,633 & 7,882 & 8,412 & 9,573 \\
\hline Coke and coal & 3,872 & 3,222 & 3,446 & 2,831 & 2,043 & 5,467 & 8,100 & 7,937 & 8,421 & 7,537 \\
\hline Crude oil and petroleum & & & & & & & & & & \\
\hline products $1 /$ & 14,312 & 15,944 & 19,245 & 19,151 & 18,527 & 12,650 & 11,516 & 13,541 & 11,591 & 12,195 \\
\hline Chemicals & 8,258 & 8,557 & 9,555 & 8,860 & 7,299 & 1,451 & 1,557 & 1,820 & 1,502 & 1,190 \\
\hline Manufactures of iron and steel & 3,746 & 4,898 & 4,705 & 3,911 & 4,462 & 4,101 & 4,276 & 3,640 & 4,249 & 8,846 \\
\hline Machinery and equipment & 692 & 734 & 809 & 787 & 723 & 1,357 & 1,408 & 1,125 & 1,272 & 1,443 \\
\hline Lumber and wood products & 5,297 & 5,928 & 7,238 & 7,146 & 5,689 & 4,165 & 4,784 & 3,792 & 4,060 & 3,747 \\
\hline Miscollaneous & 17,332 & 19,140 & 19,211 & 20,504 & 17,332 & 11,911 & 12,287 & 13,023 & 13,827 & 17,054 \\
\hline
\end{tabular}

Sources: Panama Canal Commission; and Fund staff estimates.

1/ Includes crude oil, diesel oil, fuel oil, gasoline, jet fuel, liquified natural gas, kerosene, and asphalt. 
Table 44. Panama: Travel Receipts and Expenditure, and Number of Visitors

\begin{tabular}{|c|c|c|c|c|c|}
\hline & 1994 & 1995 & 1996 & 1997 & 1998 \\
\hline \multicolumn{6}{|c|}{ (In millions of balboas) } \\
\hline Net travel receipts & 139.4 & 188.7 & 219.6 & 210.4 & 202.6 \\
\hline Travel receipts & 261.6 & 309.4 & 359.9 & 374.2 & 378.8 \\
\hline Tourists & 138.1 & 157.6 & 198.8 & 155.0 & 149.8 \\
\hline Business, official, & & & & & \\
\hline and education related travel & 59.7 & 61.3 & 53.1 & 85.8 & 93.5 \\
\hline Travelers in transit and others & 63.8 & 90.5 & 108.0 & 133.4 & 135.5 \\
\hline Expenditure of & & & & & \\
\hline Panamanians traveling abroad & -122.2 & -120.7 & -140.3 & -163.8 & -176.2 \\
\hline \multicolumn{6}{|c|}{ (In thousands) } \\
\hline Visitors 1/ & 351.2 & 380.8 & 431.8 & 413.6 & 414.5 \\
\hline Tourists & 241.3 & 268.5 & 340.3 & 263.5 & 252.3 \\
\hline $\begin{array}{l}\text { Business, official, } \\
\text { and education related travel }\end{array}$ & 109.9 & 112.3 & 91.5 & 150.1 & 162.2 \\
\hline Travelers in transit $2 /$ & 283.6 & 394.0 & 467.7 & 573.5 & 565.0 \\
\hline
\end{tabular}

Sources: Office of the Comptroller General; and Fund staff estimates.

1/ Entries into Panama excluding residents, immigrants, and persons in transit or whose destination is the Canal area.

2/ Nonresidents who spent less than 48 hours in Panama. 
Table 45. Panama: Public Sector, External Debt and Debt Service

(It millions of balboas; stocks at end of period) $1 /$

\begin{tabular}{|c|c|c|c|c|c|c|}
\hline & 1994 & 1995 & 1996 & 1997 & 1998 & $\begin{array}{r}\text { Jan-Sept } \\
1999\end{array}$ \\
\hline \multicolumn{7}{|l|}{ Stock of external debt } \\
\hline Total & $5,505.5$ & $5,890.8$ & $5,052,0$ & $5,007.5$ & $5,313,0$ & $5,631.8$ \\
\hline Multilateral & 733.0 & 764.5 & 806.0 & 933.4 & 1128.8 & 1131.8 \\
\hline $\mathrm{IMF}$ & 132.8 & 110.6 & 130.9 & 142.2 & 181.6 & 160.9 \\
\hline IBRD & 206.3 & 182.5 & 198.7 & 229.6 & 277.8 & 272.3 \\
\hline IDB & 384.8 & 462.4 & 469.7 & 554.7 & 656.6 & 678.2 \\
\hline IFAD & 9.1 & 9.0 & 6.7 & 6.9 & 12.8 & 20.4 \\
\hline Bilateral & 770.6 & 782.7 & 486.7 & 451.0 & 445.0 & 424.9 \\
\hline Paris Club 2/ & 408.4 & 400.2 & 385.4 & 333.1 & 324.1 & 311.0 \\
\hline Venezuela, Mexico & 277.2 & 299.2 & 0.0 & 0.0 & 0.0 & 0.0 \\
\hline Support group & 85.0 & 83.3 & 101.3 & 117.9 & 120.9 & 113.9 \\
\hline Commercial & $3,944.1$ & $4,299.6$ & $3,724.3$ & $3,594.2$ & $3,738.6$ & $4,074.9$ \\
\hline $\begin{array}{l}\text { Medipm- and long-term debt } \\
\text { Of which: }\end{array}$ & $3,830.0$ & $4,193.6$ & $3,724.3$ & $3,594.2$ & $3,738.6$ & $4,074.9$ \\
\hline Brady bonds & 0.0 & 0.0 & $3,227.6$ & $2,027.9$ & $1,972.2$ & $1,840.6$ \\
\hline Other bonds & 433.1 & 432.5 & 365.5 & $1,499.6$ & $1,766.4$ & $2,229.0$ \\
\hline Short-term $3 /$ & I14.1 & 106.0 & 0.0 & 0.0 & 0.0 & 0.0 \\
\hline Supplíers 4/ & 57.8 & 44.0 & 35.0 & 28.9 & 0.6 & 0.2 \\
\hline \multicolumn{7}{|l|}{ Debt-service $4 /$} \\
\hline Total & 613.9 & 652.9 & 479.5 & $1,509.8$ & 605.5 & 632.7 \\
\hline Principal & 270.3 & 262.6 & 236.0 & $1,227.5$ & 302.3 & 314.2 \\
\hline Interest & 343.6 & 390.3 & 243.5 & 282.3 & 3032 & 318.5 \\
\hline Multilateral & 146.7 & 171.5 & 170.2 & 139.2 & 120.2 & 150.8 \\
\hline Frincipal & 95.4 & 125.3 & 124.0 & 97.1 & 72.3 & 56.0 \\
\hline Interest & 51.3 & 46.2 & 46.2 & 42.1 & 47.9 & 94.8 \\
\hline Bilateral & 90.6 & 69.8 & 66.5 & 82.5 & 78.7 & 79.8 \\
\hline Principal 5/ & 49.7 & 31.2 & 30.4 & 57.2 & 58.0 & 60.3 \\
\hline Interest & 41,0 & 38.7 & 36.1 & 25.3 & 20.7 & 19.5 \\
\hline Commercial & 374.6 & 397.7 & 230.9 & $1,280.2$ & 400.3 & 401.7 \\
\hline Principal 6/ & 116.3 & 94.1 & 72.5 & $1,068.1$ & 167.6 & 197.5 \\
\hline $\begin{array}{l}\text { Interest } \\
\text { Of which: }\end{array}$ & 247.9 & 300.7 & 158.4 & 212.1 & 232.7 & 204.2 \\
\hline Short-term $7 /$ & 10.4 & 2.9 & 0.0 & 0.0 & 0.0 & 0.0 \\
\hline Suppliers & 12.3 & 16.7 & 11.8 & 7.9 & 6.3 & 0.4 \\
\hline Principal & 8.9 & 12.1 & 9.1 & 5.2 & 4.4 & 0.4 \\
\hline Interest & 3.4 & 4.6 & 2.7 & 2.7 & 1.9 & 0.0 \\
\hline
\end{tabular}

Sources: Ministry of Planning and Economic Polity; IBRD; IDB; and Fund staff estimates.

1/ Includes acerued interest arrears.

2/ Inciudes insured suppliers' credit.

3/ Noninsured suppliers' credits only.

4/ Includes imputed charges on overdue obligations.

$5 /$ Increase in 1998 is due to payment of arrears to Libyan Atab Foreign Bank.

6/ Increases in 1997 and 1998 are due to repurchases of Brady bonds.

7 Consists of interest on credit lines and on money facilities of the National Bank; the latter is excluded from interest of the nonfinaneial public sector. 
Table 46. Panama: External Public Debt Indicators

(In percent)

\begin{tabular}{lrrrrrr}
\hline & 1994 & 1995 & 1996 & 1997 & 1998 & $\begin{array}{r}\text { Sept. } \\
\end{array}$ \\
& & & & & & \\
\hline & & & & & & \\
Debt-service ratios 1/ & 34.3 & 44.3 & 30.9 & 90.3 & 34.3 & 47.6 \\
In terms of central government receipts 2/ & 17.1 & 17.7 & 15.2 & 73.5 & 17.1 & 23.7 \\
$\quad$ Principal due & 17.2 & 26.5 & 15.7 & 16.9 & 17.2 & 23.9 \\
$\quad$ Interest due & 8.1 & 8.3 & 5.8 & 17.2 & 5.7 & $\ldots$ \\
In terms of GDP & 3.5 & 3.3 & 2.9 & 14.0 & 2.4 & $\ldots$ \\
$\quad$ Principal due & 4.6 & 5.0 & 3.0 & 3.2 & 3.3 & $\ldots$ \\
$\quad$ Interest due & 20.3 & 21.9 & 15.4 & 44.4 & 16.9 & $\ldots$ \\
In terms of exports of goods and services & 8.8 & 8.8 & 7.6 & 36.1 & 7.0 & $\ldots$ \\
$\quad$ Principal due & 11.5 & 13.1 & 7.8 & 8.3 & 9.9 & $\ldots$ \\
$\quad$ Interest due & & & & & & \\
External debt ratios & 71.2 & 74.1 & 62.0 & 58.7 & 58.3 & $\ldots$ \\
External debt in terms of GDP 3/ & 19.4 & 20.3 & 16.4 & 16.4 & 12.5 & $\ldots$ \\
$\quad$ Multilateral and bilateral 4/ & & & & & & \\
$\quad$ Of which: & 1.7 & 1.4 & 1.6 & 1.6 & 2.0 & $\ldots$ \\
$\quad$ IMF & 51.7 & 54.9 & 45.6 & 41.3 & 45.8 & $\ldots$ \\
$\quad$ Other 5/ & 6.5 & 6.9 & 4.3 & 5.6 & 5.9 & 5.8 \\
Effective interest rates on total deht 6/ & & & & & & \\
Memorandum item: & 5.1 & 6.1 & 5.6 & 5.8 & 5.5 & 5.3 \\
Six-month LIBOR (year average) & & & & &
\end{tabular}

Sources: Office of the Comptroller General; National Bank of Panama; Ministry of Planning and Economic Policy; and Fund staff estimates.

1/ Debt-service ratios exclude payments due on short-term debt, but include payments due to the IMF.

2/ Central government receipts include transfers from the rest of the public sector and exclude grants.

$3 /$ Including interest arrears and short-term debt.

4/ Includes insured suppliers' credits.

$5 /$ Includes nonguaranteed suppliers' credits.

6/ Interest rate on average debt outstanding during the period. Rates are affected by variations in exchange rates. 
Table 47. Panama: Operations on Medium- and Long-Term External Debt of the Nonfinancial Public Sector Classified by Creditor 1/

(In millions of balboas)

\begin{tabular}{|c|c|c|c|c|c|c|c|}
\hline & \multicolumn{6}{|c|}{ Operations in 1994} & \multirow[b]{2}{*}{$\begin{array}{c}\text { Out- } \\
\text { stgnding } \\
12 / 31 / 94 \\
\end{array}$} \\
\hline & $\begin{array}{c}\text { Out- } \\
\text { standing } \\
12 / 31 / 93\end{array}$ & Drawings & $\begin{array}{l}\text { Amort- } \\
\text { ization } \\
\text { Due }\end{array}$ & $\begin{array}{c}\text { Interest } \\
\text { Due }\end{array}$ & Payments & $\begin{array}{c}\text { Adjust- } \\
\text { ments 2/ }\end{array}$ & \\
\hline Total & $5,213.9$ & 52.3 & 270.3 & 343,6 & 286.8 & 68.4 & $5,391.4$ \\
\hline Multilateral & 767.3 & 30.5 & 95.4 & 51.3 & 146.7 & 30.6 & 733.0 \\
\hline MMF & 115.7 & 14.0 & 1.2 & 7.6 & 8.8 & 4.2 & 132.8 \\
\hline IBRD & 244.3 & 1.4 & 53.5 & 18. 1 & 71.6 & 14.1 & 206.3 \\
\hline IDB & 397,3 & 14.6 & 39.4 & 24.7 & 64.1 & 12.3 & 384.8 \\
\hline IFAD & 10.0 & 0.5 & 1.4 & 0.8 & 2.2 & -0.1 & 9.1 \\
\hline Bilateral & 757.7 & 21.8 & 49.7 & 41.0 & 61.6 & 11.8 & 770.6 \\
\hline Paris Club $3 /$ & 417.7 & 1.8 & 17.6 & 20.8 & 38.4 & 6.5 & 408.4 \\
\hline Venezuela and Mexico & 262.1 & 0.0 & 12.1 & 16.9 & 0.0 & -1.8 & 277.2 \\
\hline Support group & 77.9 & 20.0 & 20.0 & 3.2 & 23.2 & 7.1 & 85.0 \\
\hline Commercial banks & $3,613.4$ & 0.0 & 116.3 & 247.9 & 65.7 & 34.4 & $3,830.0$ \\
\hline Medium- and long-term debt & $3,144,0$ & 0.0 & 104.4 & 224.6 & 6.0 & 34.4 & $3,397.0$ \\
\hline Bonded debt & 469.4 & 0.0 & 11.9 & 23.3 & 59.6 & 0.0 & 433.1 \\
\hline \multirow[t]{3}{*}{ Suppliers 4 I } & 75.5 & 0.0 & 8.9 & 3.4 & 12.8 & -8.3 & 57.8 \\
\hline & \multicolumn{6}{|c|}{ Operations in 1995} & \multirow[b]{2}{*}{$\begin{array}{c}\text { Out- } \\
\text { standing } \\
12 / 31 / 95 \\
\end{array}$} \\
\hline & $\begin{array}{c}\text { Out- } \\
\text { standing } \\
12 / 31 / 94 \\
\end{array}$ & Drawings & $\begin{array}{l}\text { Ancht- } \\
\text { ization } \\
\text { Due }\end{array}$ & $\begin{array}{l}\text { Interest } \\
\text { Due }\end{array}$ & Payments & $\begin{array}{l}\text { Adjust- } \\
\text { ments } 2 /\end{array}$ & \\
\hline Total & $5,391.4$ & 112.6 & 262.6 & 390.3 & 288.9 & 179.4 & $5,784.8$ \\
\hline Multilateral & 733.0 & 107.9 & 125.3 & 46.2 & 171.5 & 49.0 & 764.5 \\
\hline MF & 132.8 & 12.9 & 38.9 & 8.7 & 47.7 & 3.9 & 110.6 \\
\hline $\mathbb{E R D}$ & 206.3 & 2.8 & 43.1 & 15.5 & 58.6 & 16.5 & 182.5 \\
\hline IDB & 384.8 & 90.9 & 41.8 & 21.3 & 63.0 & 28.4 & 462.4 \\
\hline FAD & 9.1 & 1.3 & 1.5 & 0.7 & 2.3 & 0.2 & 9.0 \\
\hline Bilatera: & 770.6 & 4.7 & 31.2 & 38.7 & 44.8 & 13.6 & 782.7 \\
\hline Paris Club $3 /$ & 408.4 & 4.7 & 18.5 & 22.3 & 40.9 & 5.7 & 400.2 \\
\hline Venezuela and Mexico & 277.2 & 0.0 & 12.6 & 12.4 & 0.0 & 9.6 & 2992 \\
\hline Support group & 85.0 & 0.0 & 0.0 & 3.2 & 3.2 & -1.7 & 83.3 \\
\hline Commercial banks & $3,830.1$ & 0.0 & 94.1 & 300.7 & 55.9 & 118.5 & $4,193.6$ \\
\hline Medium- and long-tern debt & $3,397.0$ & 0.0 & 93.6 & 269.8 & 24,4 & 118.7 & $3,761.1$ \\
\hline Bonded debs & 433.1 & 0.0 & 0.5 & 310 & 31.4 & -0.2 & 432.5 \\
\hline Suppliers 4/ & 57.8 & 0.0 & 12.5 & 4.6 & 16.7 & -1.7 & 44.0 \\
\hline
\end{tabular}


Table 47. Panama: Operations on Medium- and Long-Term External Debt of the Nonfinancial Classified by Creditor (Continued) I/

(In millions of balboas)

\begin{tabular}{|c|c|c|c|c|c|c|c|}
\hline & & \multicolumn{5}{|c|}{ Operations in 1996} & \multirow[b]{2}{*}{$\begin{array}{c}\text { Ont- } \\
\text { standing } \\
12 / 31 / 96\end{array}$} \\
\hline & $\begin{array}{c}\text { Qut- } \\
\text { standing } \\
12 / 31 / 95\end{array}$ & Drawings & $\begin{array}{c}\text { Amort- } \\
\text { ization } \\
\text { Due }\end{array}$ & $\begin{array}{c}\text { Interest } \\
\text { Due } \\
\end{array}$ & Payments & $\begin{array}{l}\text { Adjust- } \\
\text { ments } 2 /\end{array}$ & \\
\hline Total & $5,784.8$ & 368.3 & 236.0 & 243.3 & 659.7 & -684.7 & $5,052.0$ \\
\hline Multilateral & 764.5 & 234.5 & 124,0 & 46.2 & 170.2 & $-69,0$ & 806.0 \\
\hline IMF & 110.6 & 75.9 & $\$ 1.9$ & 6.5 & 58.5 & -3.7 & 130.9 \\
\hline IRRD & 182.5 & 68.6 & 31.4 & 12.5 & 43.9 & -21.0 & 198.7 \\
\hline $\mathrm{DB}$ & 462.4 & 90.0 & 39.0 & 26.4 & 65.3 & -43.7 & 469.7 \\
\hline IFAD & 9.0 & 0.0 & 1.7 & 0.8 & 2.5 & -0.6 & 6.7 \\
\hline Bilateral & 782.7 & 27.8 & 30.4 & 36.1 & 247.7 & -112.1 & 486.7 \\
\hline Paris Club $3 /$ & 400.2 & 3.4 & 17.6 & 21.6 & 39.2 & -0.5 & 385.4 \\
\hline Venezuela and Mexico & 299.2 & 0.0 & 12.8 & 9.6 & 203.7 & -105.2 & 0.0 \\
\hline Support Group & 83.3 & 24.4 & 0.0 & 48 & 4.8 & -6.4 & 101.3 \\
\hline Commercial banks & $4,193.6$ & 106.0 & 72.5 & 158.4 & 230.0 & -503.7 & $3,724.3$ \\
\hline Mediunt- and long-tern debt $5 /$ & $3,761.1$ & 105,0 & 5.5 & 129.7 & 133.6 & -504.3 & $3,358.9$ \\
\hline Bonded debt & 432.5 & 0.0 & 67.0 & 28.7 & 96.4 & 0.6 & 365.4 \\
\hline \multirow[t]{3}{*}{ Suppliets $4 t$} & 44.0 & 0.0 & 9.1 & 2.7 & 11.8 & 0.1 & 35.0 \\
\hline & & \multicolumn{5}{|c|}{ Operations in 1997} & \\
\hline & $\begin{array}{c}\text { Out- } \\
\text { standing } \\
12 / 3 \mathrm{~V} / 96\end{array}$ & Drawings & $\begin{array}{c}\text { Alnort- } \\
\text { ization } \\
\text { Due } \\
\end{array}$ & $\begin{array}{c}\text { Interest } \\
\text { Due }\end{array}$ & Payments & $\begin{array}{l}\text { Adjust- } \\
\text { Inents 2/ }\end{array}$ & $\begin{array}{c}\text { Out- } \\
\text { standing } \\
12 / 31 / 97 \\
\end{array}$ \\
\hline Total & $5,052.0$ & $1,486.4$ & $1,227.5$ & 282.3 & $1,541.9$ & -271.3 & $5,007.5$ \\
\hline Multitateral & 806.0 & 247.1 & 97.1 & 42.1 & 139.2 & -22.6 & 933.4 \\
\hline DMF & 130.9 & 45.8 & 26.1 & 7.5 & 33.6 & -8.4 & 142.2 \\
\hline IBRD & 198.7 & 54.5 & 29.2 & 13.8 & 43.1 & 5.7 & 229.6 \\
\hline IDB & 469.7 & 144.6 & 39.8 & 20.3 & 60.0 & -19.9 & 554.7 \\
\hline IFAD & 6.7 & 2.2 & 1.9 & 0.5 & 2.5 & 0.0 & 6.9 \\
\hline Bilateral & 486.7 & 39.3 & 57.2 & 25.3 & 82.5 & -17.8 & 451.0 \\
\hline Paris Club 37 & 385.4 & 7.7 & 55.6 & 19.9 & 75.5 & 4.4 & 333.1 \\
\hline Venezuela and Mexico & 0.0 & 0.0 & 0.0 & 0.0 & 0.0 & 0.0 & 0.0 \\
\hline Support group & 101.3 & 31.6 & 1.6 & 5.4 & 7.0 & -13.4 & 117.9 \\
\hline Comunercia! & $3,724.3$ & $1,200.0$ & $1,058.1$ & 212.1 & $1,292.6$ & -230.0 & $3,594.2$ \\
\hline Mediunt and long-term debi st & $3,358.9$ & 0.0 & $1,002.0$ & 168.9 & $1,203,0$ & -230.2 & $2,094.6$ \\
\hline Bonded debt of & .265 .4 & $1,200.0$ & 66.0 & 43.3 & 109.3 & 0.2 & $1,499.6$ \\
\hline Suppliers A/ & 35.0 & 0.0 & 5.2 & 2.7 & 7.9 & -0.9 & 28.9 \\
\hline
\end{tabular}


Table 47. Panama: Operations on Medium- and Long-Term External Debt of the Nonfinancial Public Sector (Concluded)

(In millions of balboas)

\begin{tabular}{|c|c|c|c|c|c|c|c|}
\hline & \multirow[b]{2}{*}{$\begin{array}{c}\text { Out- } \\
\text { standing } \\
12 / 31 / 97\end{array}$} & \multicolumn{5}{|c|}{ Operations in 1998} & \multirow[b]{2}{*}{$\begin{array}{c}\text { Out- } \\
\text { standing } \\
12 / 31 / 98\end{array}$} \\
\hline & & Drawings & $\begin{array}{l}\text { Amtort- } \\
\text { ization } \\
\text { Due } \\
\end{array}$ & $\begin{array}{c}\text { Interest } \\
\text { Due }\end{array}$ & Payments & $\begin{array}{l}\text { Adjust- } \\
\text { ments 2/ }\end{array}$ & \\
\hline Total & $5,007.4$ & 703.2 & 302.3 & 303.2 & 616.2 & -85.2 & $5,312.3$ \\
\hline $\begin{array}{l}\text { Multilateral } \\
\text { DMF } \\
\text { IBRD } \\
\text { IDB } \\
\text { IFAD }\end{array}$ & $\begin{array}{r}933.4 \\
142.2 \\
229.6 \\
554.7 \\
6.9\end{array}$ & $\begin{array}{r}273.1 \\
41.5 \\
91.3 \\
133.1 \\
7.2\end{array}$ & $\begin{array}{r}72.3 \\
13.3 \\
25.9 \\
31.2 \\
1.9\end{array}$ & $\begin{array}{r}47.9 \\
8.1 \\
14.5 \\
24.7 \\
0.6\end{array}$ & $\begin{array}{r}120.2 \\
21.4 \\
40.4 \\
55.9 \\
2.4\end{array}$ & $\begin{array}{r}-6.2 \\
11.2 \\
-18.0 \\
0.0 \\
0.6\end{array}$ & $\begin{array}{r}1,128.0 \\
181.6 \\
277.0 \\
656.6 \\
12.8\end{array}$ \\
\hline $\begin{array}{l}\text { Bilateral } \\
\text { Peris Club 3/ } \\
\text { Venezuela and Mexico } \\
\text { Support Group }\end{array}$ & $\begin{array}{r}451.0 \\
333.1 \\
0.0 \\
117.9\end{array}$ & $\begin{array}{r}97.0 \\
82.5 \\
0.0 \\
14.5\end{array}$ & $\begin{array}{r}58.0 \\
56.4 \\
0.0 \\
1.6\end{array}$ & $\begin{array}{r}20.7 \\
16.9 \\
0.0 \\
3.8\end{array}$ & $\begin{array}{r}105.4 \\
100.0 \\
0.0 \\
5.4\end{array}$ & $\begin{array}{r}-18.3 \\
-8.4 \\
0.0 \\
-9.9\end{array}$ & $\begin{array}{r}445.0 \\
324.1 \\
0.0 \\
120.9\end{array}$ \\
\hline $\begin{array}{l}\text { Comumercial } \\
\text { Medium- and long-term debt } \\
\text { Bonded debt } 6 /\end{array}$ & $\begin{array}{l}3,594.2 \\
2,094.6 \\
1,499.6\end{array}$ & $\begin{array}{r}333.0 \\
0.0 \\
333.0\end{array}$ & $\begin{array}{r}167.6 \\
102.1 \\
65.5\end{array}$ & $\begin{array}{r}232.7 \\
98.1 \\
134.6\end{array}$ & $\begin{array}{l}384.3 \\
184.7 \\
199.6\end{array}$ & $\begin{array}{r}-36.9 \\
-35.7 \\
-1.2\end{array}$ & $\begin{array}{l}3,738.7 \\
1,972.3 \\
1,766.4\end{array}$ \\
\hline \multirow[t]{2}{*}{ Suppliers 4/ } & 28.9 & 0.0 & 4.4 & 1.9 & 6.3 & -23.9 & 0,6 \\
\hline & $\begin{array}{c}\text { Out- } \\
\text { standing } \\
12 / 31 / 98\end{array}$ & Drawings & $\begin{array}{l}\text { Amations in } \\
\text { ization } \\
\text { Due } \\
\end{array}$ & $\begin{array}{l}\text { January-s } \\
\text { Interest } \\
\text { Due }\end{array}$ & eptember 15 & $\begin{array}{l}\text { Adjust- } \\
\text { ments } 2 /\end{array}$ & $\begin{array}{l}\text { Out- } \\
\text { standing } \\
9 / 30 / 99\end{array}$ \\
\hline Total & $5,312.3$ & 619.2 & 314.2 & 318.5 & 609.5 & -8.7 & $5,631.8$ \\
\hline $\begin{array}{l}\text { Multifateral } \\
\text { IMF } \\
\text { IBRD } \\
\text { IDB } \\
\text { IFAD }\end{array}$ & $\begin{array}{r}1,128.0 \\
181.6 \\
277.0 \\
656.6 \\
12.8\end{array}$ & $\begin{array}{r}75.5 \\
0.0 \\
24.7 \\
41.1 \\
9.7\end{array}$ & $\begin{array}{r}56.0 \\
11.5 \\
18.9 \\
23.6 \\
2.0\end{array}$ & $\begin{array}{r}94.8 \\
4.6 \\
35.2 \\
54.4 \\
0.6\end{array}$ & $\begin{array}{r}138.6 \\
16.1 \\
54.1 \\
65.6 \\
2.8\end{array}$ & $\begin{array}{r}-27.9 \\
-9.2 \\
-10.5 \\
-8.3 \\
0.1\end{array}$ & $\begin{array}{r}1,131.8 \\
160.9 \\
272.3 \\
578.2 \\
20.4\end{array}$ \\
\hline $\begin{array}{l}\text { Bilateral } \\
\text { Paris Clut } 3 / \\
\text { Venezuela and Mexico } \\
\text { Support Group }\end{array}$ & $\begin{array}{r}445.0 \\
324.1 \\
0.0 \\
120.9\end{array}$ & $\begin{array}{r}43.6 \\
43.6 \\
0.0 \\
0.0\end{array}$ & $\begin{array}{r}60.3 \\
53.2 \\
0.0 \\
7.1\end{array}$ & $\begin{array}{r}19.5 \\
15.7 \\
0.0 \\
3.8\end{array}$ & $\begin{array}{r}83.9 \\
68.9 \\
0.0 \\
15.0\end{array}$ & $\begin{array}{r}0.7 \\
-3.5 \\
0.0 \\
4.2\end{array}$ & $\begin{array}{r}424.9 \\
311.0 \\
0.0 \\
113.9\end{array}$ \\
\hline $\begin{array}{l}\text { Commercial } \\
\text { Mediurn- and long-term debt } \\
\text { Bonded debt } 6 /\end{array}$ & $\begin{array}{l}3,738.7 \\
1,972.3 \\
1,766.4\end{array}$ & $\begin{array}{r}500.1 \\
0.5 \\
499.6\end{array}$ & $\begin{array}{r}197.5 \\
163.9 \\
33.6\end{array}$ & $\begin{array}{r}204.2 \\
95.3 \\
108.9\end{array}$ & $\begin{array}{l}386.8 \\
244.7 \\
142.1\end{array}$ & $\begin{array}{l}18.7 \\
22.5 \\
-3.8\end{array}$ & $\begin{array}{l}4,074.9 \\
1,845.9 \\
2,229.0\end{array}$ \\
\hline Suppliers $4 f$ & 0.6 & 0.0 & 0.4 & 0.0 & 0.2 & -0.2 & 0.2 \\
\hline
\end{tabular}

Sources: Ministry of Planning and Economic Policy; National Bank of Panama; $\mathbb{M F} ; \mathrm{DB}$; $\mathbb{B R R}$; and Fund staff estimates.

1/ Excluding shor-term debt. Figures include interest arrears.

$2 /$ Adjustments due to valuation changes, data tevisions, and/or debt reduction.

3/ Includes insured suppliers' credit.

4/ Noninsured suppliers' credits only.

5/ Drawing refers to short-term debl refinanced in January 1996 into medium-tenn debt. it aiso includes commercial bank debt converted into Brady bonds in 1996 and arrears to Libya.

bi Euro bonds (US\$500 million) and Global bonds (US\$700 nillion) were issued in 1997, 10-year bonds (US\$300 million) were issued in 1998; and an additional global bonds (US\$500 million) were issued in 1999 Were issued in 1999 
Table 48. Panama: Arrears on Public Sector External Debt Service

(In millions of balboas; end of period)

\begin{tabular}{|c|c|c|c|c|c|c|c|c|c|c|c|c|}
\hline & \multicolumn{2}{|c|}{1994} & \multicolumn{2}{|c|}{1995} & \multicolumn{2}{|c|}{1996} & \multicolumn{2}{|c|}{1997} & \multicolumn{2}{|c|}{1998} & \multicolumn{2}{|c|}{ September 1999} \\
\hline & Pтincipal & Interest & Principal & Interest & Principal & Interest & Principal & Interest & Principal & Interest & Principal & Interest \\
\hline Total & $1,927.2$ & $1,598.9$ & $1,954.8$ & $1,983.3$ & 51.5 & 45.8 & 21.3 & 19.9 & 0.0 & 0.0 & 0.0 & 0.0 \\
\hline $\begin{array}{l}\text { Commercial banks } \\
\text { Of which: }\end{array}$ & $1,800.2$ & $1,478.6$ & $1,817.9$ & $1,841.1$ & 48.8 & 44.0 & 20.0 & 19.2 & 0.0 & 0.0 & 0.0 & 0.0 \\
\hline Short-term debt $1 /$ & 72.0 & 42.1 & 28.1 & 34.0 & 20.0 & 15.2 & 20.0 & 19.2 & 0.0 & 0.0 & 0.0 & 0.0 \\
\hline Bonded debt & 2.3 & 0.7 & 2.5 & 0.7 & 1.4 & 1.1 & 0.0 & 0.0 & 0.0 & 0.0 & 0.0 & 0.0 \\
\hline Mexico and Venezuela & 121.2 & 117.2 & 133.1 & 140.5 & 0.0 & 0.0 & 0.0 & 0.0 & 0.0 & 0.0 & 0.0 & 0.0 \\
\hline Suppliers & 3.5 & 2.4 & 1.3 & 1.0 & 1.3 & 0.7 & 1.3 & 0.7 & 0.0 & 0.0 & 0.0 & 0.0 \\
\hline
\end{tabular}

Sources: Ministry of Planning and Economic Policy; IMF; IDB; IBRD; and Fund staff estimates.

1/ Consists of credit lines to public enterprises and money facilities of the National Bank. 